\title{
Flavor and collider signatures of asymmetric dark matter
}

\author{
Ian-Woo Kim ${ }^{1,2}$ and Kathryn M. Zurek ${ }^{1}$ \\ ${ }^{1}$ Michigan Center for Theoretical Physics, University of Michigan, Ann Arbor, Michigan 48109, USA \\ ${ }^{2}$ CERN, Theory Division, 1211 Geneva 23, Switzerland \\ (Received 21 October 2013; published 19 February 2014)
}

\begin{abstract}
We consider flavor constraints on, and collider signatures of, asymmetric dark matter (ADM) via higher dimension operators. In the supersymmetric models we consider, $R$-parity-violating (RPV) operators carrying $B-L$ interact with $n$ dark matter particles $X$ through an interaction of the form $W=X^{n} \mathcal{O}_{B-L}$, where $\mathcal{O}_{B-L}=q \ell d^{c}, u^{c} d^{c} d^{c}, \ell \ell e^{c}$. This interaction ensures that the lightest ordinary supersymmetric particle is unstable to decay into the $X$ sector, leading to a higher multiplicity of final state particles and reduced missing energy at a collider. Flavor-violating processes place constraints on the scale of the higher dimension operator, impacting whether the LOSP decays promptly. While the strongest limitations on RPV from $n-\bar{n}$ oscillations and proton decay do not apply to ADM, we analyze the constraints from meson mixing, $\mu-e$ conversion, $\mu \rightarrow 3 e$ and $b \rightarrow s \ell^{+} \ell^{-}$. We show that these flavor constraints, even in the absence of flavor symmetries, allow parameter space for prompt decay to the $X$ sector, with additional jets and leptons in exotic flavor combinations. We study the constraints from existing $8 \mathrm{TeV}$ LHC Supersymmetry (SUSY) searches with (i) 2-6 jets plus missing energy and (ii) 1-2 leptons, 3-6 jets plus missing energy, comparing the constraints on ADM-extended supersymmetry with the usual supersymmetric simplified models.
\end{abstract}

DOI: 10.1103/PhysRevD.89.035008

\section{INTRODUCTION}

The notion that dark matter (DM) may be related to the baryon asymmetry originates from a time almost as early as the weakly interacting massive particle (WIMP) paradigm itself $[1,2]$. In these models, a mechanism sets the DM and baryon asymmetries such that $n_{X}-n_{\bar{X}} \sim n_{b}-n_{\bar{b}}$, where $n_{X}, n_{\bar{X}}$ are the DM and anti-DM number densities, and $n_{b}, n_{\bar{b}}$ are the baryon and antibaryon asymmetries. Since the ratio of DM to baryon densities is observed to be $\rho_{\mathrm{DM}} / \rho_{B} \sim 5$, this suggests $m_{X} \sim 5 m_{p} \simeq 5 \mathrm{GeV}$, where $m_{X}$ is the DM mass and $m_{p}$ is the proton mass. Thus in these models, the natural mass scale for the DM is around 1-10 times the proton mass, significantly below the weak scale.

The idea that the DM and baryon densities have a common mechanism setting their densities is a simple and compelling framework. The challenge for a model of DM that relates the DM and baryon asymmetries is, however, that it must satisfy the many requirements from our observations of the weak scale and below. Many of the earliest models, especially those making use of electroweak sphalerons [3-5], have become highly constrained by these observations, particularly those from LEP, making models of DM relating the DM and baryon asymmetries observationally less than compelling.

Employing ideas from hidden sector model building [6], the asymmetric dark matter (ADM) paradigm [7] showed how to evade these constraints by making use of higher dimension operators $\mathcal{O}_{B-L}$ which carry no Standard Model (SM) gauge charge but carry $B-L$. These operators are connected to the DM sector via higher dimension operators
PACS numbers: 12.60.Jv, 11.30.Hv, 12.60.-i, 95.35.+d

$$
\mathcal{O}_{\mathrm{ADM}}=\frac{\mathcal{O}_{B-L} \mathcal{O}_{X}}{M^{n+m-4}}
$$

where $\mathcal{O}_{B-L}$ has dimension $m$ and $\mathcal{O}_{X}$ has dimension $n$. The operators in Eq. (1.1) share a primordial matterantimatter asymmetry between the visible and DM sectors, realizing the relationship $n_{X}-n_{\bar{X}} \sim n_{b}-n_{\bar{b}}$. For a review and list of references of DM models employing the higher dimension operators, see [8].

ADM can be embedded within supersymmetry (SUSY), which stabilizes the ADM particle via $R$ parity and limits the types of operators in the superpotential. The simplest (lowest dimension) superpotential operators for $\mathcal{O}_{B-L}$ are the $R$-parity-violating (RPV) operators

$$
W_{B-L}=\ell H, \quad u^{c} d^{c} d^{c}, \quad q \ell d^{c}, \quad \ell \ell e^{c},
$$

where $\ell$ is a SM lepton doublet, $H$ the Higgs doublet, $u^{c}, d^{c}$ right-handed antiquarks, $e^{c}$ a right-handed charged antilepton, and $q$ is a quark doublet. The simplest form of superpotential operators for $\mathcal{O}_{X}$ is $X$, so the simplest ADM interactions take the form

$$
W_{\mathrm{ADM}}=X \ell H, \quad \frac{X u_{i}^{c} d_{j}^{c} d_{k}^{c}}{M_{i j k}}, \quad \frac{X q_{i} \ell_{j} d_{k}^{c}}{M_{i j k}}, \frac{X \ell_{i} \ell_{j} e_{k}^{c}}{M_{i j k}},
$$

where now we have explicitly included a flavor index $i, j, k$ on the generic scale of the operator $M$.

These interactions are centrally important for the collider phenomenology of ADM-extended SUSY models. The 


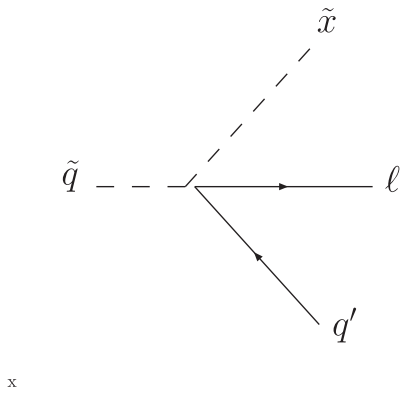

(a)

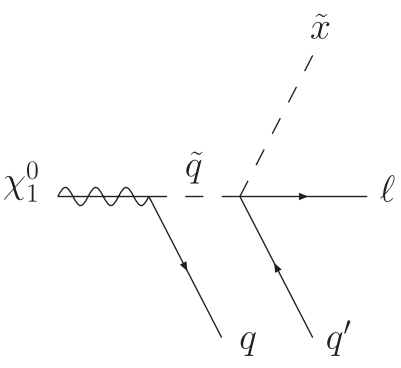

(b)
FIG. 1. Decay of a squark LOSP directly through the interaction of Eq. (1.3), and decay of a neutralino LOSP through an off-shell squark for $q \ell d^{c}$ models. Here, the quark flavors $q$ and $q^{\prime}$ are generically different. $\tilde{x}$ denotes the scalar component of the ADM supermultiplet $X$. Decay of (a) a slepton LOSP and (b) a neutralino LOSP through an off-shell squark is also given by the same diagrams, trading a squark and a lepton with a slepton and a quark, respectively.

interactions in Eq. (1.3) induce decay of the lightest ordinary supersymmetric particle (LOSP) to the DM particle, through the processes shown in Fig. 1. This implies that, in comparison to the Minimal Supersymmetric Standard Model (MSSM), the missing energy is reduced while the multiplicity of final state particles increases, so experimental sensitivity to ADM models can be very different in SUSY searches at the LHC. A number of theories, such as hidden valleys [6,9], MeV DM [10], RPV [11] and stealth SUSY [12,13], have already aimed to evade SUSY constraints by reducing the missing energy and increasing the number of final state particles. While ADM models have similar structure in their collider signatures, they also have a potentially wider range of flavor signatures.

Whether such signatures are realized at a collider depends on whether the LOSP is unstable to decay to the $X$ sector before the LOSP exits the detector. The lifetime of the LOSP is set by its nature (e.g., squark, neutralino or slepton), by the supersymmetric spectrum, and, most importantly, by the scale $M$ of the operator. The scale $M$ can be strongly constrained by flavor physics, in a way similar to RPV. Taken alone, without additional flavor structure, the RPV operators in Eq. (1.2) are known to have disastrous effects in, e.g., proton decay and neutronantineutron $(n-\bar{n})$ oscillations [14].

There are, however, several important differences between ADM operators and RPV operators. First of all, with the presence of $\mathcal{O}_{X}, R$ parity is no longer violated, if the operator $\mathcal{O}_{X}$ itself carries $R$ parity of -1 . This new $R$ parity stabilizes the lightest $R$-parity odd scalar, $\tilde{x}$, of supermultiplet $X$. Second, DM now effectively carries baryon or lepton number so that globally $B$ and $L$ are not violated. That forbids $n-\bar{n}$ oscillations as well as proton decay (when the $X$ fermion is heavier than the proton). For certain types of $X$ sectors, the DM can induce proton decay, but it must be catalyzed by the DM. For this to happen frequently enough to be observable, the scale $M$ must be quite low, around a $\mathrm{TeV}[15,16]$. Thus the worst of the usual constraints on RPV is lifted for ADM.

Depending on the flavor structure of the model and the UV completion, however, the scales $M_{i j k}$ in Eq. (1.3) are still constrained by meson oscillations, by flavor changing processes such as $b \rightarrow s \ell^{+} \ell^{-}$, and by various types of lepton flavor violation such as $\mu \rightarrow e$ conversion and $\mu \rightarrow 3 e$.

The flavor structure (and the corresponding constraints on the scale of the operator) thus has important implications for the collider signatures of ADM. As we will see, for example, the lifetime of a pure Bino neutralino at the LHC through the operator $X q \ell d^{c}$ and an intermediate right-handed $d$ squark is roughly

$$
\begin{aligned}
c \tau \sim & (200 \mathrm{~mm}) \\
& \times\left(\frac{M}{100 \mathrm{TeV}}\right)^{2}\left(\frac{m_{\tilde{q}}}{500 \mathrm{GeV}}\right)^{4}\left(\frac{100 \mathrm{GeV}}{m_{\chi^{0}}}\right)^{7},
\end{aligned}
$$

where $m_{\tilde{q}}$ is the mass of the intermediate squark and $M$ is the scale of the $X q \ell d^{c}$ operator. Depending on the constraints on $M$ and $m_{\tilde{q}}, \chi^{0}$ may be collider stable though cosmologically unstable. Therefore, it is important to consider constraints from a displaced secondary vertex search for generic ADM models. Previously, some lifetime estimates have been made by using naive dimensional analysis [7,17], but it is desirable to refine the displaced vertex analysis.

The goal of this paper is to study the flavor structure and constraints on ADM and their implications for collider searches for SUSY. We compute the flavor constraints on the scale $M$ of the operator, relate these constraints to the lifetime of the LOSP, and derive constraints on the ADMextended MSSM from standard SUSY searches. Unlike many recent efforts to lift constraints on RPV operator coefficients through flavor structures [18,19], we will assume no flavor symmetry but rather examine the range of possible signatures that could arise in a general flavor structure. Note that the flavor constraints we place on DM in ADM models will have applications to many models with flavorful DM [20-24] because the UV completion of the ADM models we consider contains some of the same interactions.

The outline of our paper is as follows. In Sec. II, we carry out a thorough analysis of the flavor structure of all three ADM models $\left(q \ell d^{c}, u^{c} d^{c} d^{c}, \ell \ell e^{c}\right)$ for the simplest UV completions (except for the $\ell H$ model which is essentially a model with a right-handed neutrino). We extract constraints on the general scale $M$ of the ADM operator from various flavor processes. We highlight the results in Sec. II and provide details of our flavor analysis in Appendix A. Next, in Sec. III, we examine the implications of the flavor constraints on $M$ for the LOSP lifetime at the LHC. We give 
details in Appendix B of exact expressions for the lifetime of the LOSP through three- and four-body decays [for which Eq. (1.4) is only an approximate proxy]. We show that prompt, displaced, and collider stable signatures are all possible, consistent with flavor constraints, even in the absence of a flavor symmetry. Then, in Sec. IV, we carry out a detailed analysis of the constraints on this model from existing searches assuming prompt LOSP decays. We compare the constraints in the standard SUSY searches against those for ADM for $8 \mathrm{TeV}$ LHC analyses utilizing (i) 0 leptons plus 2-6 jets plus missing energy and (ii) $1-2$ leptons plus 3-6 jets plus missing energy. We thus lay firm groundwork for a more exhaustive analysis of SUSY ADM signatures at the LHC in the future, before concluding in Sec. V.

\section{OPERATORS AND THEIR FLAVOR CONSTRAINTS}

We begin our study by discussing the UV completions for each higher dimension operator in Eq. (1.3), assessing the impact from flavor constraints on the scale $M$ of the operators in $W_{\mathrm{ADM}}$. As we will see in Sec. III, a careful computation of the lifetime of the LOSP shows that only when the scale of the operator in Eq. (1.3) is $M \gtrsim 100 \mathrm{TeV}$ will the decay of a neutralino LOSP be collider stable, or displaced, at the LHC (though the details depend on the supersymmetric spectrum of the model). Thus for phenomenological study of prompt decays at the LHC, we are mostly interested in flavor constraints that require $M \gtrsim m_{M} / \lambda^{2} \gtrsim 10-100 \mathrm{TeV}$, where $M$ is determined from a UV completion by $m_{M}$, the mass of the mediator being integrated out to generate the operator, and $\lambda^{2}$, a product of couplings of that mediator to SM states and the DM. We summarize the results for constraints derived from meson oscillations, $\mu-e$ conversion, $\mu \rightarrow 3 e$ and $b \rightarrow s \ell^{+} \ell^{-}$ including $B_{s} \rightarrow \ell^{+} \ell^{-}$in this section, and refer the reader to Appendix A for the details of our computations. $K-\bar{K}$ mixing provides, in many cases (except for the $X \ell \ell e^{c}$ operator), the strongest constraint.

We emphasize that we take a conservative approach without assuming a flavor structure, since there are many ways to relax flavor constraints by imposing a flavor structure on the model. For example, since both meson oscillations and lepton flavor constrain products of couplings to different generations, if the couplings to one of the generations are much larger than to the other generations, the constraints will be considerably relaxed. In the case of meson oscillations the usefulness of this change is somewhat limited, however, since rotating from a flavor basis to the mass basis will induce couplings to the other generations which are generically not small unless the flavor and mass bases are closely aligned (which would constitute a tuning in the absence of a flavor symmetry). In such cases, a flavor symmetry can alleviate these constraints. Therefore, our results on flavor constraints and the corresponding discussion of displaced vertices from LOSP decay must be taken as conservative. Even without the assumption of a flavor symmetry, we will find that prompt flavor-violating decays of the LOSP are still possible at the LHC. In addition, deriving constraints in the absence of a flavor symmetry leaves open the interesting possibility for exotic flavor signatures at the LHC.

\section{A. $X q \ell d^{c}$}

We begin by analyzing the $X q \ell d^{c}$ operator, assuming only one flavor of DM:

$$
W_{\mathrm{ADM}}=\frac{X q_{i} \ell_{j} d_{k}^{c}}{M_{i j k}}
$$

There are three UV completions at the renormalizable level:

$$
\begin{gathered}
W^{(D)}=\lambda_{X D}^{i} X d_{i}^{c} D+\lambda_{D}^{i j} D^{c} q_{i} \ell_{j}+m_{D} D D^{c}, \\
W^{(L)}=\lambda_{X L}^{i} X \ell_{i} L^{c}+\lambda_{L}^{i j} L q_{i} d_{j}^{c}+m_{L} L L^{c} \\
W^{(Q)}=\lambda_{X Q}^{i} X q_{i} Q^{c}+\lambda_{Q}^{i j} Q \ell_{i} d_{j}^{c}+m_{Q} Q Q^{c},
\end{gathered}
$$

where $i, j, k$ are generation indices. Note that the effective scale of $W$ is determined by

$$
M_{i j k}=m_{M} /\left(\lambda_{X M}^{i} \lambda_{M}^{j k}\right)
$$

for a mediator $M=D L, Q$ with mass $m_{M}$. This relation also holds for the UV completion, given an appropriate mediator $M$, of the $X u^{c} d^{c} d^{c}$ and $X \ell \ell e^{c}$ operators, as will be shown in the next subsections. In these expressions, as throughout this paper, a lowercase letter indicates a SM field and an uppercase letter represents exotic heavy states, which are integrated out to generate the higher dimension operator. Note that we define fields in the mass eigenstate basis here. For simplicity, we consider only one flavor of DM, as well as a single pair of heavy mediator fields $\left(D, D^{c}\right),\left(L, L^{c}\right)$ or $\left(Q, Q^{c}\right)$. If we extend this simple model with multiple DM flavors or multiple mediator states, we have more freedom in assigning a flavor structure that could lift some of the flavor constraints that we study, but we do not pursue this direction. We also assume only one of the UV completions is dominant, and we will label the UV completion by the state which is being integrated out. Our results do not qualitatively change if we consider mixed UV completions.

We consider the constraints on $X q \ell d^{c}$ derived from $K-\bar{K}, D-\bar{D}, B-\bar{B}$ mixing, $\mu-e$ conversion, $\mu \rightarrow 3 e$ and $b \rightarrow s \ell^{+} \ell^{-}$in turn.

\section{Meson mixing}

Both tree- and loop-level processes give rise to meson mixing through the UV completions for the operator in 


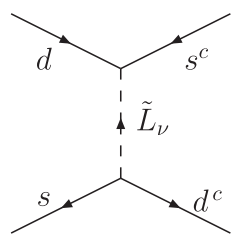

(a)

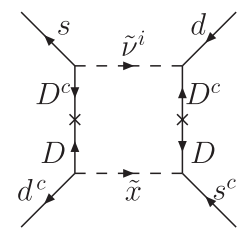

(b)

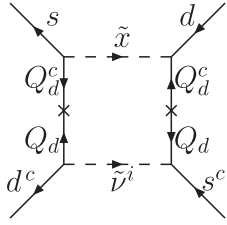

(c)

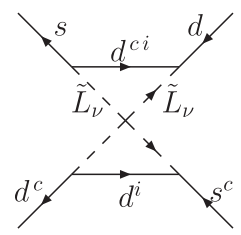

(d)

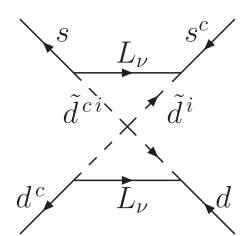

(e)

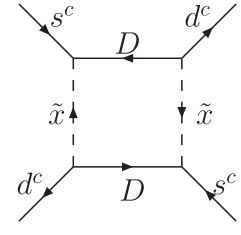

(f)

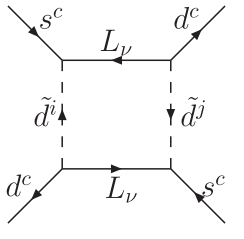

$(\mathrm{g})$

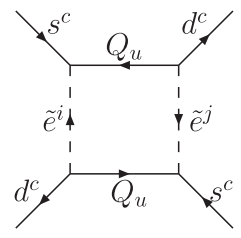

(h)

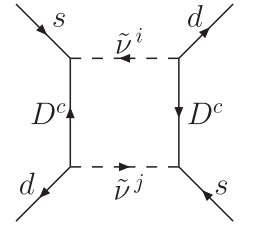

(i)

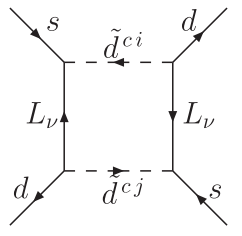

(j)

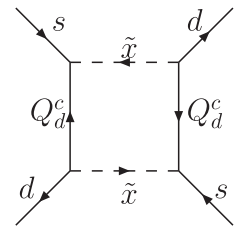

$(\mathrm{k})$

FIG. 2. Diagrams contributing to $K-\bar{K}$ mixing in $X q \ell d^{c}$ models. Diagrams (a)-(e) contribute to $\left(\bar{s}_{R} d_{L}\right)\left(\bar{s}_{L} d_{R}\right)$. Diagrams (f)-(h) contribute to $\left(\bar{s}_{R} \gamma^{\mu} d_{R}\right)^{2}$. Diagrams (i)-(k) contribute to $\left(\bar{s}_{L} \gamma^{\mu} d_{L}\right)^{2}$. For $\left(\bar{s}_{L, R} \gamma^{\mu} d_{L, R}\right)^{2}$, we only show a representative diagram for each UV completion. Here, we use two-component spinor notation to reduce confusion.

Eq. (2.1). Sample processes are shown in Fig. 2. While the tree-level processes, in principle, give rise to a stronger constraint on the mediator mass, they do not constrain the DM coupling $\lambda_{X M}$ to the UV particle $M$, nor do they constrain all UV completions (the $D$ and $Q$ UV completions are untouched by the tree-level constraint). An exhaustive compilation of the couplings constrained by $K-\bar{K}, D-\bar{D}$ and $B-\bar{B}$ mixing is given in Table $\mathrm{V}$ in Appendix A; we highlight the conclusions here.

Meson mixing is most strongly constraining for the operator $\left(\bar{s}_{R} d_{L}\right)\left(\bar{s}_{L} d_{R}\right) / \Lambda^{2}$, where $K-\bar{K}$ mixing gives $\Lambda \gtrsim 1.8 \times 10^{4} \mathrm{TeV}[25]$. For the $L$ UV completion, Fig. 2(a) will generate $K-\bar{K}$ mixing at tree level,

$$
\begin{gathered}
\frac{\lambda_{L}^{12} \lambda_{L}^{12}}{m_{\tilde{L}}^{2}}\left(\bar{s}_{R} d_{L}\right)\left(\bar{s}_{L} d_{R}\right), \quad \text { for }(L), \\
\frac{m_{\tilde{L}}}{\sqrt{\lambda_{L}^{12} \lambda_{L}^{12}}} \geq 1.8 \times 10^{4} \mathrm{TeV} .
\end{gathered}
$$

For $B_{d}$ and $B_{s}$ meson mixing, we also have similar treelevel diagrams generating left-right operators, which have stringent constraints as summarized in Appendix A, Table V. Nevertheless, we note that only the $L$ UV completion is constrained for a very limited combination of couplings by meson mixing at tree level.

Loop diagrams, on the other hand, probe a wider array of flavor-changing couplings, since any of the superpartner flavors may appear in the loop. In some cases, they also probe precisely the combination of couplings $t$ hat enters into $M_{i j k}$, which ultimately determines whether decays are prompt or displaced at the LHC. Contributions to $\mathcal{O}_{A}=\left(\bar{s}_{R} \gamma^{\mu} d_{R}\right)^{2} / \Lambda_{A}^{2},\left(\bar{s}_{L} \gamma^{\mu} d_{L}\right)^{2} / \Lambda_{A}^{2}$ and $\mathcal{O}_{B}\left(\bar{s}_{L} d_{R}\right)\left(\bar{s}_{R} d_{L}\right) / \Lambda_{B}^{2}$ occur, and the loop functions which characterize the constraints are detailed in Appendix A. In the limit that the fermions and the scalars in the loop have common masses $m_{F}$ and $m_{\varphi}$, respectively, the amplitude simplifies considerably:

$$
\begin{aligned}
\frac{1}{\Lambda_{A}^{2}} \sim \frac{\lambda^{4}}{64 \pi^{2}}\left(\frac{m_{F}^{2}+m_{\phi}^{2}}{\left(m_{F}^{2}-m_{\phi}^{2}\right)^{2}}-\frac{2 m_{F}^{2} m_{\phi}^{2}}{\left(m_{F}^{2}-m_{\phi}^{2}\right)^{3}} \log \left(\frac{m_{F}^{2}}{m_{\phi}^{2}}\right)\right) \\
\frac{1}{\Lambda_{B}^{2}} \sim \frac{\lambda^{4} m_{F}^{2}}{16 \pi^{2}}\left(-\frac{2}{\left(m_{F}^{2}-m_{\phi}^{2}\right)^{2}}+\frac{m_{F}^{2}+m_{\phi}^{2}}{\left(m_{F}^{2}-m_{\phi}^{2}\right)^{3}} \log \left(\frac{m_{F}^{2}}{m_{\phi}^{2}}\right)\right),
\end{aligned}
$$

where $\lambda^{2}$ represents the appropriate combination of couplings shown in Table $\mathrm{V}$ for amplitudes having structure corresponding to operators $A$ and $B$. The constraint on $\Lambda_{B} \gtrsim 2 \times 10^{4} \mathrm{TeV}$ is strongest and corresponds to a limit on the parameters of the UV completion $m_{M} / \lambda^{2} \gtrsim$ $1000 \mathrm{TeV}$. While it is not a universal constraint on all the couplings to all generations, as can be seen in Table V, it is the most severe constraint on $M_{i j k}$. 


\section{2. $\mu-e$ conversion, $\mu \rightarrow 3 e, B_{s} \rightarrow \ell^{+} \ell^{-}$and $b \rightarrow s \ell^{+} \ell^{-}$}

Lepton flavor violation may also constrain the UV completions of the ADM operators with heavy states $Q$, $L, D$. The strongest constraints are derived from $\mu-e$ conversion and are summarized in Appendix A 2 in Table V. At tree level, the $D$ UV completion and the $Q$ UV completion have contributions to $\mu-e$ conversion through the diagrams shown in Fig. 3, resulting in the operators

$$
\begin{aligned}
- & \frac{1}{2} \frac{\lambda_{D}^{11} \lambda_{D}^{12}}{m_{\tilde{D}}^{2}}\left(\bar{e}_{L} \gamma^{\rho} \mu_{L}\right)\left(\bar{u}_{L} \gamma_{\rho} u_{L}\right), \quad \text { for }(D), \\
- & \frac{1}{2} \frac{\lambda_{Q}^{11} \lambda_{Q}^{21}}{m_{\tilde{Q}}^{2}}\left(\bar{e}_{L} \gamma^{\rho} \mu_{L}\right)\left(\bar{d}_{R} \gamma_{\rho} d_{R}\right), \quad \text { for }(Q),
\end{aligned}
$$

where we rearrange spinors using the Fierz identities, $\quad\left(\bar{e}_{L} u_{R}^{c}\right)\left(\bar{u}_{R}^{c} \mu_{L}\right)=-\frac{1}{2}\left(\bar{e}_{L} \gamma^{\rho} \mu_{L}\right)\left(\bar{u}_{L} \gamma_{\rho} u_{L}\right) \quad$ and $\left(\bar{e}_{L} d_{R}\right)\left(\bar{d}_{R} \mu_{L}\right)=-\frac{1}{2}\left(\bar{e}_{L} \gamma^{\rho} \mu_{L}\right)\left(\bar{d}_{R} \gamma_{\rho} d_{R}\right)$. The branching ratio $(\mathrm{Br})$ of $\mu-e$ conversion is obtained for the various nuclei and can be translated into the value for $\mathrm{Al}$, $\operatorname{Br}_{\mu N \rightarrow e N}(Z=13) \leq 10^{-12}$ (see Appendix A 2). We then derive the constraint

$$
\frac{m_{\tilde{D}}}{\sqrt{\lambda_{D}^{11} \lambda_{D}^{12}}} \geq 290 \mathrm{TeV}, \quad \frac{m_{\tilde{Q}}}{\sqrt{\lambda_{Q}^{11} \lambda_{Q}^{21}}} \geq 210 \mathrm{TeV} .
$$

The number of coefficients constrained by the tree-level process is, however, limited. On the other hand, loop-level contributions, as also shown in Fig. 3, constrain all three UV completions for various combinations of couplings. These are detailed exhaustively in Table VI in Appendix A 2. At loop level, the constraints on $M$ from $\mu-e$ conversion are at the level of 10-100 $\mathrm{TeV}$ and therefore not important from the point of view of displaced vertices at the LHC. $\mu \rightarrow e \gamma$ and $\mu \rightarrow 3 e$ appear only at loop level and are not strong constraints either. We detail the constraints from $\mu \rightarrow 3 e$ in Appendix A, Table VIII. While not significant for the $q \ell d^{c}$ model, $\mu \rightarrow 3 e$ will become important for the $\ell \ell e^{c}$ model.

At tree level, we also have contributions to $b-s$ conversion with a pair of leptons, as for example in $B_{s} \rightarrow$ $\mu^{+} \mu^{-}$. The processes are shown in Figs. 4(a) and 4(b),

$$
\begin{aligned}
- & \frac{1}{2} \frac{\lambda_{D}^{32} \lambda_{D}^{22}}{m_{\tilde{D}}^{2}}\left(\bar{\mu}_{L} \gamma^{\rho} \mu_{L}\right)\left(\bar{s}_{L} \gamma_{\rho} b_{L}\right), \quad \text { for }(D), \\
- & \frac{1}{2} \frac{\lambda_{Q}^{23} \lambda_{Q}^{22}}{m_{\tilde{Q}}^{2}}\left(\bar{\mu}_{L} \gamma^{\rho} \mu_{L}\right)\left(\bar{s}_{R} \gamma_{\rho} b_{R}\right), \quad \text { for }(Q),
\end{aligned}
$$

where we again rearrange spinors using the Fierz identities, similarly to Eq. (2.8). In our analysis, we use a combined experimental bound [26] for $B_{s} \rightarrow \mu^{+} \mu^{-}$from the 2011 LHC data [26-29]: $\operatorname{Br}\left(B_{s} \rightarrow \mu^{+} \mu^{-}\right)<4.2 \times 10^{-9}$. Note that, recently, the first evidence of $B_{s} \rightarrow \mu^{+} \mu^{-}$was found at $\mathrm{LHCb}$, and the result is consistent with $\mathrm{SM}$ [30]; the results have been updated with the 2012 LHC data [31,32].

We can also constrain the scale of four-fermion effective operators through the process $b \rightarrow s \ell^{+} \ell^{-}$[33]. The

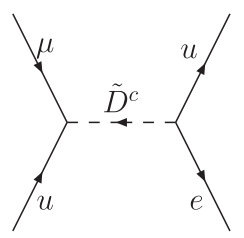

(a)

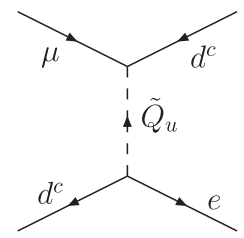

(b)

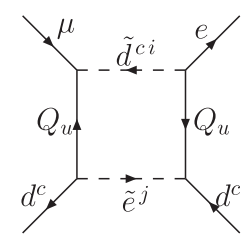

(c)

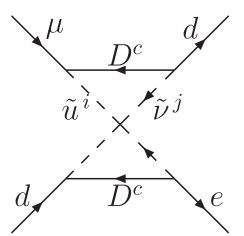

(d)

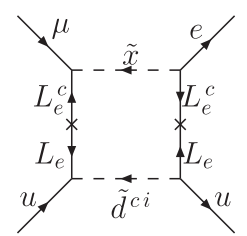

(e)

FIG. 3. Diagrams contributing to $\mu-e$ conversion in $X q \ell d^{c}$ models. We show only a typical one-loop diagram for each UV completion in (c)-(e), as well as tree diagrams (a) and (b). For a more complete set of diagrams, see Appendix A.

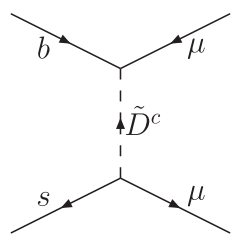

(a)

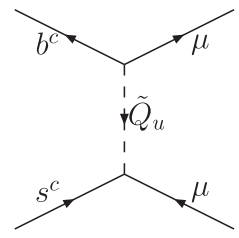

(b)

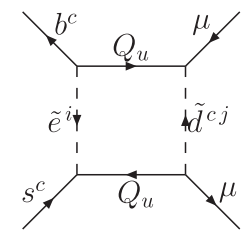

(c)

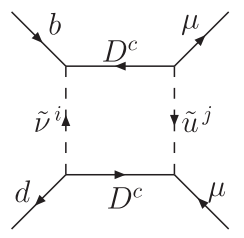

(d)

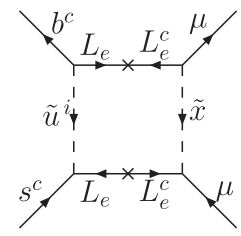

(e)

FIG. 4. Diagrams contributing to $B_{s} \rightarrow \mu^{+} \mu^{-}$in the $q \ell d^{c}$ model. We show only a representative one-loop diagram for each UV completion in (c)-(e), as well as tree diagrams (a) and (b). The additional box diagrams are shown in Fig. 23. For other $b-s$ transitions such as $b \rightarrow s \ell^{+} \ell^{-}$, one can easily obtain contributing Feynman diagrams by properly changing the external states in the above diagrams. 
tree-level constraints lead to (see Appendix A, Table VII for details)

$m_{D} / \sqrt{\lambda_{D}^{3 \ell} \lambda_{D}^{2 \ell}}>32 \mathrm{TeV}$ for strongest, $>11 \mathrm{TeV}$

for weakest,

$m_{Q} / \sqrt{\lambda_{Q}^{2 \ell} \lambda_{Q}^{2 \ell}}>45 \mathrm{TeV}$ for strongest, $>11 \mathrm{TeV}$

for weakest,

where $\ell=1,2$ denotes the electron and the muon, respectively, for the lepton final states, and we show both the strongest constraint and the weakest constraint since the constraint varies depending on the sign of the coupling, and whether it is real or imaginary. While only the $D$ and $Q \mathrm{UV}$ completions contribute at tree level, all UV completions contribute at one loop, as shown in Figs. 4(c), 4(d) and 4(e), though the loop suppression implies that this constraint will be weak. The details can be found in Appendix A 3.

\section{Summary of constraints for $X q \ell d^{c}$}

There are many combinations of couplings constrained in Tables V-VIII, but it is important to see the overarching patterns.

(i) The strongest constraints are on the operator $\left(\bar{s}_{L} d_{R}\right)\left(\bar{d}_{L} s_{R}\right) / \Lambda^{2}$, and they give rise roughly to a constraint $M \gtrsim 1000 \mathrm{TeV}$ for the UV completions via $D$ and $Q$. Since $M$ is the quantity which enters into the lifetimes in Fig. 1, it directly enters into the discussion of displaced vertices in the next section. These constraints can be eased and $M$ lowered if one or both of the quarks in the decay of $\tilde{\chi}_{0} \rightarrow q q \ell$ is of the third generation. Note that the constraints are equally strong on all lepton flavors.

(ii) The UV completions via $L$ are less constrained. The strongest constraint on $M$ is derived from the geometric mean of the $K-\bar{K}$ mixing and $\mu \rightarrow e \gamma$, which results in $M \gtrsim 10 \mathrm{TeV}$. The constraints can be relaxed somewhat if the lepton in $\tilde{\chi}_{0} \rightarrow q q \ell$ is $\tau$ or one of the quarks is of the third generation.

In Sec. III we give precise formulas for the LOSP lifetime as a function of $M$, thus mapping the flavor constraints onto displaced vertex signatures for ADM.

Before examining the collider signatures, however, we complete our discussion of the flavor constraints with an examination of the other ADM operators.

\section{B. $X u^{c} d^{c} d^{c}$}

Considering next the $X u^{c} d^{c} d^{c}$ operator, the UV completions are

$W^{(U)}=\lambda_{X U}^{i} X u_{i}^{c} U+\frac{1}{2} \lambda_{U}^{i j} U^{c} d_{i}^{c} d_{j}^{c}+m_{U} U U^{c}$,

$W^{(D)}=\lambda_{X D}^{i} X d_{i}^{c} D+\lambda_{D}^{i j} D^{c} u_{i}^{c} d_{j}^{c}+m_{D} D D^{c}$,

where $i, j$ are flavor indices.

Similar to the case of $X q \ell d^{c}$, the combinations of the couplings which are constrained are shown in Table V. Because all fields involved are right handed, the strongest constraint from $\left(\bar{s}_{L} d_{R}\right)\left(\bar{d}_{L} s_{R}\right)$ is eliminated, and more modest constraints on $m_{M} / \lambda^{2}$ between 10 and $100 \mathrm{TeV}$ result. Note in addition that $\lambda_{X U}^{3}$ is the only coupling which remains unconstrained. A variety of other processes from $q_{i} \rightarrow q_{j} q \bar{q}$ meson decays will constrain $\lambda^{2} / m_{M}^{2}$, similar to $\mu-e$ conversion or $B_{s} \rightarrow \mu^{+} \mu^{-}$constraints on the $q \ell d^{c}$ model. These constraints are, however, rather weak. Since no constraints on $M_{i j k}$ exceed $100 \mathrm{TeV}$, prompt decays of the LOSP are unconstrained by flavor.

\section{C. $\boldsymbol{X} \ell \ell \boldsymbol{e}^{c}$}

Lastly, we consider the UV completions for $X \ell \ell e^{c}$,

$$
\begin{gathered}
W^{(L)}=\lambda_{X L}^{i} X \ell_{i} L^{c}+\lambda_{L}^{i j} L \ell_{i} e_{j}^{c}+m_{L} L L^{c}, \\
W^{(E)}=\lambda_{X E}^{i} X e_{i}^{c} E+\frac{1}{2} \lambda_{E}^{i j} E^{c} \ell_{i} \ell_{j}+m_{E} E E^{c},
\end{gathered}
$$

where $i$ and $j$ are again flavor indices.

$$
\text { 1. } \boldsymbol{\mu} \rightarrow \mathbf{3 e}
$$

The $L$ UV completion of the $\ell \ell e^{c}$ model has a tree-level contribution to the $\mu \rightarrow e e e$ process as shown in Fig. 5, which leads to effective operators:

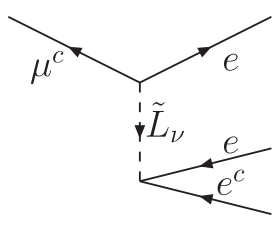

(a)

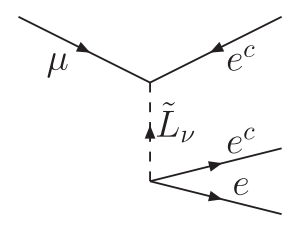

(b)

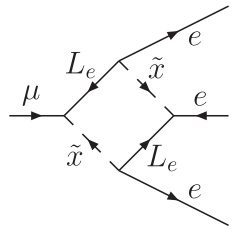

(c)

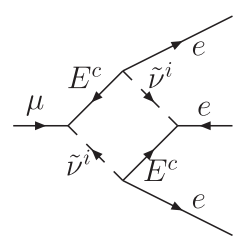

(d)

FIG. 5. Diagrams contributing to $\mu \rightarrow e^{+} e^{-} e^{-}$in $\ell \ell e^{c}$ models. We show only a typical diagram for loop contributions for each UV completion in (c) and (d), as well as tree diagrams (a) and (b). 
TABLE I. Three-body decay modes, for various LOSP choices, and their lifetime factor $F^{(3-\text { body })}$ [from Eq. (3.1)] in $q \ell d^{c}, u^{c} d^{c} d^{c}$ and $\ell \ell e^{c}$ models. $\Lambda_{*}$ is the scale of $M_{i j k}$ that gives rise to a displaced vertex at $1 \mathrm{~mm}$ with $m_{\mathrm{LOSP}}=1 \mathrm{TeV}$.

- For $X q \ell d^{c}$ :

LOSP

\begin{tabular}{cc} 
Decay mode & $\left(F^{(3-\text { body })}\right)^{-1}(\mathrm{~mm})$ \\
$\tilde{u}_{i} \rightarrow d_{j} e_{k}^{+} \tilde{x}^{*}$ & $4.71 \times 10^{-5}$ \\
$\tilde{d}_{i} \rightarrow d_{j} \bar{\nu}_{k} \tilde{x}^{*}$ & $4.71 \times 10^{-5}$ \\
$\tilde{d}_{i}^{c} \rightarrow \bar{u}_{j} e_{k}^{+} \tilde{x}^{*} \bar{d}_{j} \bar{\nu}_{k} \tilde{x}^{*}$ & $2.36 \times 10^{-5}$ \\
$\tilde{e}_{i}^{-} \rightarrow \bar{u}_{j} d_{k} \tilde{x}^{*}$ & $1.57 \times 10^{-5}$ \\
$\tilde{\nu}_{i} \rightarrow \bar{d}_{j} d_{k} \tilde{x}^{*}$ & $1.57 \times 10^{-5}$ \\
\hline
\end{tabular}

$\begin{array}{cc}\text { Decay mode } & \left(F^{(3-\mathrm{body})}\right)^{-1}(\mathrm{~mm}) \\ \tilde{u}_{i} \rightarrow d_{j} e_{k}^{+} \tilde{x}^{*} & 4.71 \times 10^{-5} \\ \tilde{d}_{i} \rightarrow d_{j} \bar{\nu}_{k} \tilde{x}^{*} & 4.71 \times 10^{-5} \\ \tilde{d}_{i}^{c} \rightarrow \bar{u}_{j} e_{k}^{+} \tilde{x}^{*}, \bar{d}_{j} \bar{\nu}_{k} \tilde{x}^{*} & 2.36 \times 10^{-5} \\ \tilde{e}_{i}^{-} \rightarrow \bar{u}_{j} d_{k} \tilde{x}^{*} & 1.57 \times 10^{-5} \\ \tilde{\nu}_{i} \rightarrow \bar{d}_{j} d_{k} \tilde{x}^{*} & 1.57 \times 10^{-5}\end{array}$

$\Lambda_{*}(\mathrm{TeV})$

Left-handed $u$ squark

Left-handed $d$ squark

Right-handed $d$ squark

Left-handed slepton

Sneutrino $\mathrm{v}$

- For $X u^{c} d^{c} d^{c}$ :

LOSP

Decay mode

Right-handed $u$ squark

Right-handed $d$ squark

$\tilde{u}_{i}^{c} \rightarrow d_{j} d_{k} \tilde{x}^{*}(j \neq k)$

$\tilde{d}_{i}^{c} \rightarrow u_{j} d_{k} \tilde{x}^{*}(i \neq k)$

$\left(F^{(3-\text { body })}\right)^{-1}(\mathrm{~mm})$
$2.36 \times 10^{-5}$
$2.36 \times 10^{-5}$

$4.61 \times 10^{5}$

$4.61 \times 10^{5}$

$6.51 \times 10^{5}$

$7.96 \times 10^{5}$

$7.96 \times 10^{5}$

\begin{tabular}{lc}
\hline \hline For $\ell \ell e^{c}:$ & \\
LOSP & Decay mode \\
\hline Left-handed slepton & $\tilde{e}_{i}^{-} \rightarrow \bar{\nu}_{j} e_{k}^{-} \tilde{x}^{*}(i \neq j)$ \\
Right-handed slepton & $\tilde{e}_{i}^{c+} \rightarrow \bar{\nu}_{j} e_{k}^{+} \tilde{x}^{*}, e_{j}^{+} \bar{\nu}_{k} \tilde{x}^{*}(j \neq k)$ \\
Sneutrino & $\tilde{\nu}_{i} \rightarrow e_{j}^{+} e_{k}^{-} \tilde{x}^{*}(i \neq j)$ \\
\hline
\end{tabular}

$$
-\frac{1}{2} \frac{\lambda_{L}^{11} \lambda_{L}^{12}}{m_{\tilde{L}}^{2}}\left(\bar{e}_{L} \gamma^{\rho} e_{L}\right)\left(\bar{e}_{R} \gamma_{\rho} \mu_{R}\right)-\frac{1}{2} \frac{\lambda_{L}^{21} \lambda_{L}^{11}}{m_{\tilde{L}}^{2}}\left(\bar{e}_{R} \gamma^{\rho} e_{R}\right)\left(\bar{e}_{L} \gamma_{\rho} \mu_{L}\right)
$$

The branching ratio $\operatorname{Br}(\mu \rightarrow 3 e)$ is smaller than $10^{-12}$, and thus the mass $m_{\tilde{L}}$ and $\lambda$ 's involved are constrained to be

$$
\frac{m_{\tilde{L}}}{\sqrt{\tilde{\lambda}^{2}}} \geq 87 \mathrm{TeV}, \text { where } \tilde{\lambda}^{2}=\lambda_{L}^{11} \sqrt{\left(\lambda_{L}^{12}\right)^{2}+\left(\lambda_{L}^{21}\right)^{2}} .
$$

Loop processes are also constrained, and we detail their contributions in Table VIII. The conclusion of the detailed results in Appendix $\mathrm{A}$ is that no process constrains $M>100 \mathrm{TeV}$, and therefore, the LOSP decays at the LHC will be prompt.

\section{PROMPT VERSUS DISPLACED VERTEX LOSP DECAYS AT COLLIDERS}

In this section, we connect the flavor constraints on the scale $M_{i j k}$ summarized in the previous section to the lifetime of the LOSP decaying through the ADM operator Eq. (1.3) in the processes of Fig. $1 .^{1}$ LOSPs that participate in $W_{\mathrm{ADM}}$, such as squarks or sleptons, can decay directly into two SM particles and the ADM through the $W_{\mathrm{ADM}}$ operator, as in the left panel of Fig. 1. If the LOSP does not appear in $W_{\mathrm{ADM}}$ directly (e.g., neutralinos or charginos), they will decay through an off-shell squark or slepton, as in the right panel of Fig. 1. Three-body decay and four-body decay

\footnotetext{
${ }^{1}$ This figure shows explicitly the decay for the $X q \ell d^{c}$ operator, though for the $u^{c} d^{c} d^{c}$ and $\ell \ell e^{c}$ models, the decay processes are similar.
}

of the LOSP lead to completely different lifetime scales and thus result in very different constraints from displaced vertex measurement at the LHC. In Appendix B, we derive the LOSP decay width for general three- and four-body decay as shown in Fig. 24 with various group representations for participating particles. We summarize the results of Appendix B here.

For three-body LOSP decay of a squark or a slepton, the secondary vertex displacement $c \tau$ is of the form

$$
(c \tau)^{-1}=F^{(3-\text { body })} \times\left(\frac{m_{\mathrm{LOSP}}}{100 \mathrm{GeV}}\right)^{3} \times\left(\frac{100 \mathrm{TeV}}{M_{i j k}}\right)^{2}\left(\mathrm{~mm}^{-1}\right)
$$

where $m_{\text {LOSP }}$ is the LOSP mass and $F^{(3-\text { body })}$ is the coefficient that can be calculated from Eq. (B7). Here, we ignore the SM particle masses, which, in particular, exclude top-quark final state cases. Note that we also ignore the ADM mass in Eq. (3.1) since the squark mass and the slepton mass must be much larger than a typical ADM mass around $10 \mathrm{GeV}$ due to other direct collider constraints. We use the millimeter unit for the displacement since the detectors at the LHC can roughly resolve the displaced vertex up to a millimeter.

Assuming that the LOSP decays through only one dominant coupling $1 / M_{i j k}$ that does not involve the third generation, ${ }^{2}$ we list the three-body LOSP decays for each

\footnotetext{
${ }^{2}$ The third generation complicates the general discussion because the top-quark mass cannot be ignored and the thirdgeneration squarks generally have a large mixing. We leave the third-generation-specific scenarios for future work.
} 


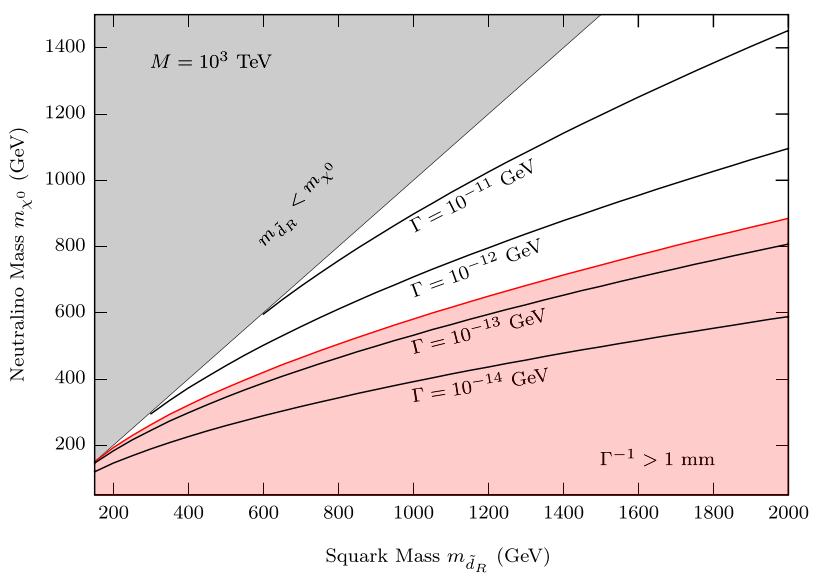

(a) $M=1000 \mathrm{TeV}$

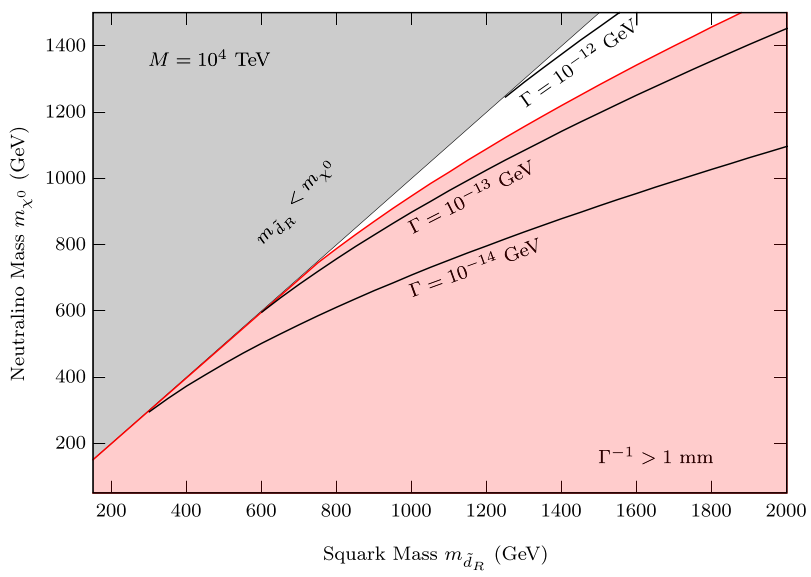

(b) $M=10^{4} \mathrm{TeV}$

FIG. 6 (color online). Neutralino decay width in the $\left(m_{\tilde{d}_{R}}, m_{\chi^{0}}\right)$ plane in the $q \ell d^{c}$ model for (a) $M=1000 \mathrm{TeV}$ and (b) $M=10^{4} \mathrm{TeV}$, where $M$ is the effective mass scale of the dominant $X q \ell d^{c}$ operator. In the lower-right shaded region (red), the neutralino will leave a displaced vertex at the LHC, defined by where the lifetime is longer than a millimeter.

superpotential operator and obtain $F^{(3-\text { body })}$ for each case in Table I. One can easily see that the displacement is generically prompt for $\mathcal{O}(100 \mathrm{GeV})$ LOSP mass for the $M_{i j k}$ scale around the flavor constraints in the previous section. To show it clearly, we list the scale of $M_{i j k}$ that gives a displaced vertex at $1 \mathrm{~mm}$ with a $1 \mathrm{TeV}$ LOSP in Table I.

For four-body LOSP decay, the displacement is given by the following expression if we assume that a contribution from a single intermediate particle $\varphi$ dominates:

$$
\begin{aligned}
(c \tau)^{-1}= & F^{(4-\text { body })} \times\left(\frac{100 \mathrm{TeV}}{M_{i j k}}\right)^{2} \times\left(\frac{500 \mathrm{GeV}}{m_{\phi}}\right)^{4} \times\left(\frac{m_{\text {LOSP }}}{100 \mathrm{GeV}}\right)^{7} \\
& \times \frac{1}{x^{5}}\left[\left(10 x^{3}-120 x^{2}+120 x\right)+60(1-x)(2-x) \log (1-x)\right]\left(\mathrm{mm}^{-1}\right),
\end{aligned}
$$

where $\varphi$ is the intermediate squark or slepton with mass $m_{\varphi}$ and $x=\left(m_{\mathrm{LOSP}} / m_{\phi}\right)^{2}$. The coefficient $F^{(4-\text { body })}$ can be determined by Eq. (B10). Note that the expression in the second line in Eq. (3.2) is reduced to $\sim(1+x)$ in the limit of $x \ll 1$.

We have many possibilities for such four-body LOSP decay in the ADM models. Since gauginos and Higgsino do not participate in the operators $X q \ell d^{c}, X u^{c} d^{c} d^{c}$ and $X \ell \ell e^{c}$, the neutralino, chargino and gluino LOSPs will decay through intermediate squarks or sleptons/sneutrinos. While the gluino LOSP decay is simply determined from QCD interactions through intermediate squarks, the neutralino LOSP and chargino LOSP depend on the details of the mixing. In general, several off-shell intermediate particle exchanges can contribute with similar size. An exhaustive study for this is beyond the scope of this paper. Instead, we only consider special cases to show typical constraints.

In Fig. 6, we consider the case with a pure Bino (neutralino) LOSP with one light right-handed $d$ squark $\tilde{d}^{c}$. We assume that only the first-generation coupling
$1 / M_{111}$ for the $X q_{i} \ell_{j} d_{k}^{c}$ operator is dominant. In this scenario, we obtain $\left(F^{(4-\text { body })}\right)^{-1}=2.04 \times 10^{2} \mathrm{~mm}$. Figure 6(a) shows the neutralino decay width contour in the $\left(m_{\tilde{d}^{c}}, m_{\chi^{0}}\right)$ plane with $M \equiv M_{111}=1000 \mathrm{TeV}$, and Fig. 6(b) shows one for $M=10^{4} \mathrm{TeV}$. While displaced vertices result over a significant fraction of the parameter space, the decays are prompt over much of the parameter space even for high choices of $M$, naively consistent with the flavor constraints even in the absence of flavor symmetries.

In the case of displaced decays, by searching for the displaced vertex, we can clearly identify DM creation inside the detector and probe the nature of the DM directly at the LHC. Thus, displaced vertex searches are very important for ADM searches at the LHC. In the case of prompt decays, however, one basic question is how ADM models fare when subjected to the usual supersymmetric searches. In the next section we compare the constraints from two standard searches for SUSY against those obtained in ADM when the LOSP is unstable to decay. 


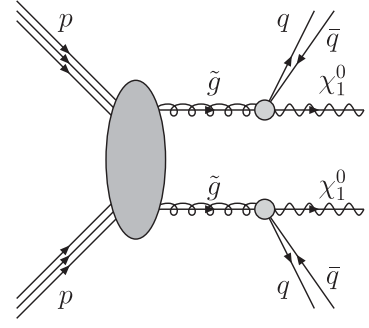

(a) gluino-gluino

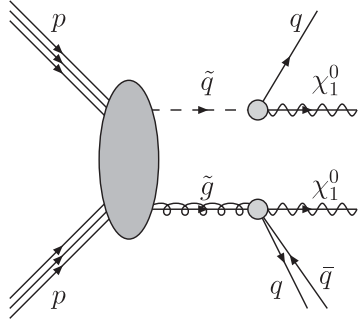

(b) squark-gluino

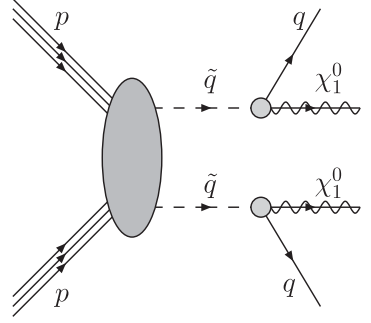

(c) squark-squark

FIG. 7. Relevant processes: (a) gluino-gluino (b) squark-gluino and (c) squark-squark pair production for ATLAS 0-lepton + 2-6-jet + MET analysis for the simplified model Sim0.

\section{LHC CONSTRAINTS}

In order to compare the standard searches for SUSY against those obtained in ADM, we consider two ATLAS analyses with $20.3 \mathrm{fb}^{-1}$ of data at $8 \mathrm{TeV}$. We have chosen the ATLAS, instead of CMS, analyses in this study since the collaboration quotes the $95 \%$ confidence limit $S_{\exp }^{95}$ on the number of events from new physics, once the cuts of the analysis have been applied. This allows us to simulate the SM plus new physics and easily extract the constraint by simply taking the difference with a simulation having the SM only. We utilize the following.

(1) An analysis with a lepton veto, 2-6 hard jets and high missing transverse energy (MET) $E_{T}^{\text {miss }}$ [34]. We will refer to this analysis as the

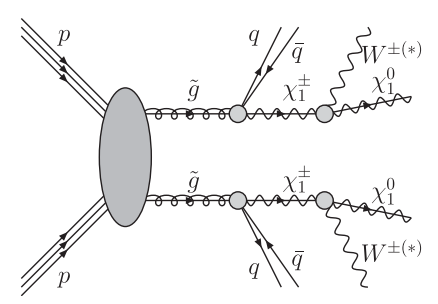

(a) gluino-gluino (Sim1g)

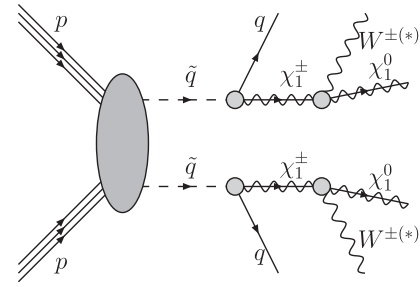

(b) squark-squark (Sim1q)
FIG. 8. Relevant processes for ATLAS 1-2-lepton +3-6-jet + MET analysis for simplified models (a) Simlg and (b) Simlq. "0-lepton + 2-6-jet + MET analysis" (or "0-lepton analysis" for short).

(2) An analysis with 1 or 2 leptons, 3-6 hard jets and high $E_{T}^{\text {miss }}$ [35]. We will refer to this analysis as the "1-2-lepton + 3-6-jet + MET analysis" (or "1-2lepton analysis" for short).

Both of these analyses are the most standard SUSY searches for typical gluino or first- or second-generation squark pair production modes in $R$-parity conserving SUSY scenarios. We aim to compare the ADM models with the ordinary SUSY models, represented by simplified models [36,37], with the relevant processes shown in Figs. 7, 8. The simplified models are designed for ease of model-independent comparison among different $R$ parity conserving SUSY scenarios.

In the case of ADM, both the 0-lepton and 1-2-lepton analyses are well targeted to the $q \ell d^{c}$ model, as shown in the processes of Figs. 9, 10. For $u^{c} d^{c} d^{c}$, the 0-lepton + 2-6-jet + MET search is effective through the processes shown in Figs. 9, 10, where additional jets from the LOSP decay are traded for a reduced missing energy cut. Other ATLAS and CMS analyses may also be relevant for constraining certain ADM models (such as the ATLAS and CMS high jet multiplicity analyses [38,39] for the $u^{c} d^{c} d^{c}$ model). We have not explored these constraints here, instead choosing a representative sample which utilizes the most standard types of SUSY analyses. In

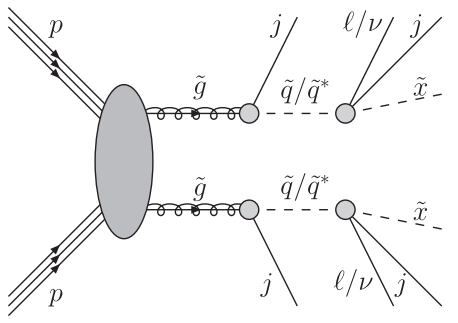

(a) gluino-gluino

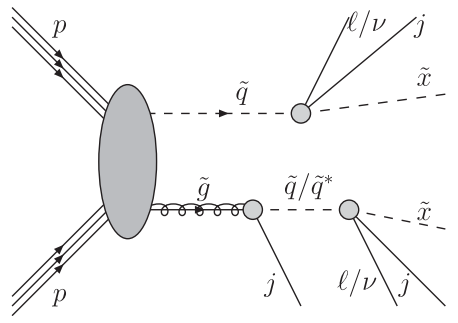

(b) squark-gluino

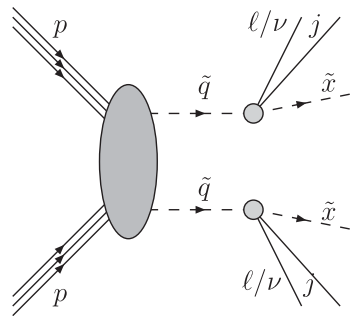

(c) squark-squark

FIG. 9. Relevant processes: (a) gluino-gluino, (b) squark-gluino and (c) squark-squark pair production for the squark LOSP case in the $q \ell d^{c}$ model. Here $\ell / \nu$ implies a lepton or a neutrino which is almost equally produced in squark decay. The $u^{c} d^{c} d^{c}$ model has the same diagrams with a lepton or neutrino replaced by a jet in the final decays of squarks. 


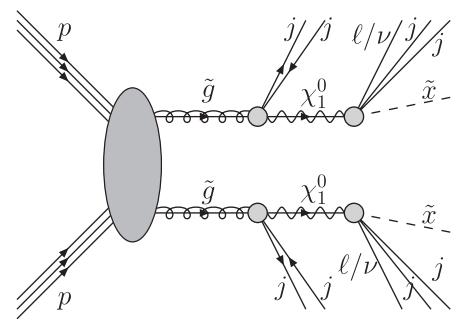

(a) gluino-gluino

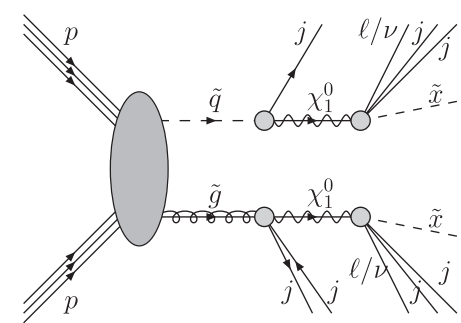

(b) squark-gluino

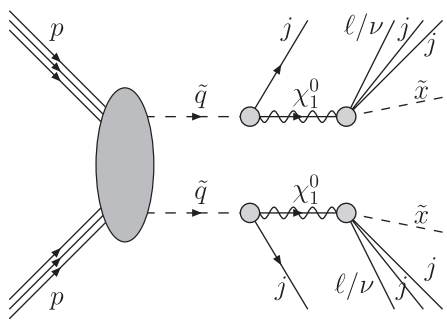

(c) squark-squark

FIG. 10. Relevant processes: (a) gluino-gluino, (b) squark-gluino and (c) squark-squark pair production for the neutralino LOSP case in the $q \ell d^{c}$ model. $\ell / \nu$ implies a lepton or a neutrino. The four-body neutralino decay is through an off-shell squark as shown in Fig. 1 (b). The $u^{c} d^{c} d^{c}$ model has the same diagrams with a lepton or neutrino replaced by a jet in the neutralino decay.

addition, we do not consider gluino and slepton/sneutrino LOSPs, or the constraints on $\ell \ell e^{c}$ operators. A more exhaustive analysis including these other cases is very interesting for future work.

\section{A. Analyses}

We briefly review the $8 \mathrm{TeV}$ ATLAS 0-lepton + 2-6-jet + MET analysis and 1-2-lepton + 3-6-jet + MET analyses and how these analyses may constrain ADM $q \ell d^{c}$ and $u^{c} d^{c} d^{c}$ models, in comparison to the simplified models that are utilized in the original ATLAS analysis. We also summarize the definition of the observables and the notation used in the analyses in Appendix C.

\section{0-lepton $+2-6-j e t+M E T$ analysis}

The ATLAS 0-lepton + 2-6-jet + MET analysis with $20.3 \mathrm{fb}^{-1}$ at $\sqrt{s}=8 \mathrm{TeV}$ is summarized in Table II. The analysis is designed to maximize the discovery potential for gluino and squark pair production with decays to neutralinos and jets. Events with signal leptons are vetoed. Events are classified into 10 nonexclusive channels: AL, AM, BM, BT, CM, CT, D, EL, EM and ET, where A, B, C, D and E imply the number of jets $N=2,3,4,5$ and 6 , respectively, and L, M and T imply loose, medium and tight cuts on the effective mass scale, respectively.

For comparison, we consider the simplified model process shown in Fig. 7. The simplified model has the gluino $\tilde{g}$, the lightest neutralino $\chi_{1}^{0}$ and all the left-handed squarks $\tilde{q}_{i L}$ and right-handed squarks $\tilde{q}_{i R}$ of the first and second generations with degenerate mass. In this model, the only SUSY particle production channel is gluino/squark pair production through the SUSY QCD processes. The gluino decays through $\tilde{g} \rightarrow q \tilde{q}^{(*)} \rightarrow q q \chi_{1}^{0}$ with $100 \%$ branching ratio, where the intermediate squark $\tilde{q}^{(*)}$ can be either on shell or off shell depending on mass parameters, and a squark directly decays into the neutralino and a quark. To distinguish this from other simplified models that we consider for the 1-2-lepton analysis, we denote this model "Sim0."

The most important features that will be relevant for distinguishing the constraints on the ADM model versus the simplified model are as follows: (i) $E_{T}^{\text {miss }}>160 \mathrm{GeV}$, which we will see rather dramatically reduces the acceptance of the ADM models; (ii) $N_{\text {jet }}$ with $p_{T}>60 \mathrm{GeV}$, which improves the acceptance for the ADM models with a large number of jets; (iii) $m_{\mathrm{eff}}$ and $E_{T}^{\mathrm{miss}} / m_{\mathrm{eff}}$, both of which improve the acceptance of the ADM model over the

TABLE II. A summary of the ATLAS 0-lepton + 2-6-jet + MET analysis at $8 \mathrm{TeV}, 20.3 \mathrm{fb}^{-1}$. This table is an excerpt from Table 1 and Table 4 in [34].

\begin{tabular}{|c|c|c|c|c|c|c|c|c|c|c|}
\hline \multirow[t]{2}{*}{ Requirement } & \multicolumn{2}{|c|}{ A (2 jets) } & \multicolumn{2}{|c|}{ B (3 jets) } & \multicolumn{2}{|c|}{$\begin{array}{r}\text { Channel } \\
\mathrm{C} \text { (4 jets) }\end{array}$} & \multirow{2}{*}{$\begin{array}{c}\mathrm{D}(5 \text { jets }) \\
\ldots\end{array}$} & \multicolumn{3}{|c|}{ E (6 jets) } \\
\hline & $\mathrm{L}$ & $\mathrm{M}$ & M & $\mathrm{T}$ & $\mathrm{M}$ & $\mathrm{T}$ & & $\mathrm{L}$ & M & $\mathrm{T}$ \\
\hline Common & \multicolumn{10}{|c|}{$E_{T}^{\text {miss }}>160 \mathrm{GeV}, p_{T}\left(j_{1}\right)>130 \mathrm{GeV}, p_{T}\left(j_{2}\right)>60 \mathrm{GeV}$} \\
\hline$p_{T}\left(j_{i}\right)(i \geq 3)$ & \multicolumn{10}{|c|}{$>60 \mathrm{GeV}$ for $i=3 \ldots N$ for $N$-jet channel } \\
\hline$\Delta \phi\left(\right.$ jet,$\left.E_{T}^{\text {miss }}\right)>$ & \multicolumn{4}{|c|}{$0.4(i=[1,2,(3)])$} & \multicolumn{6}{|c|}{$0.4(i=[1,2,3]), 0.2$ for $p_{T}\left(j_{i}\right)>40 \mathrm{GeV}$} \\
\hline$E_{T}^{\mathrm{miss}} / m_{\mathrm{eff}}(N j)>$ & 0.2 & $-(\mathrm{a})$ & 0.3 & 0.4 & 0.25 & 0.25 & 0.2 & 0.15 & 0.2 & 0.25 \\
\hline$m_{\text {eff }}($ incl. $)[\mathrm{GeV}]>$ & 1000 & 1600 & 1800 & 2200 & 1200 & 2200 & 1600 & 1000 & 1200 & 1500 \\
\hline $\begin{array}{l}S_{\text {exp }}^{95} \\
\text { Error }\end{array}$ & $\begin{array}{r}1135.0 \\
+332.7 \\
-291.5\end{array}$ & $\begin{array}{l}42.7 \\
+15.5 \\
-11.4\end{array}$ & $\begin{array}{l}17.0 \\
+6.6 \\
-4.6\end{array}$ & $\begin{array}{c}5.8 \\
+2.9 \\
-1.8\end{array}$ & $\begin{array}{c}72.9 \\
+23.6 \\
-18.0\end{array}$ & $\begin{array}{l}3.3 \\
+2.1 \\
-1.2\end{array}$ & $\begin{array}{l}13.6 \\
+5.1 \\
-3.5\end{array}$ & $\begin{array}{c}57.3 \\
+20.0 \\
-14.4\end{array}$ & $\begin{array}{l}21.4 \\
+7.6 \\
-5.8\end{array}$ & $\begin{array}{c}6.5 \\
+3.0 \\
-1.9\end{array}$ \\
\hline
\end{tabular}


TABLE III. A summary of the ATLAS 1-2-lepton + 3-6-jet + MET analysis at $8 \mathrm{TeV}, 20.3 \mathrm{fb}^{-1}$. Part 1: Soft lepton events. Here, the leading lepton $p_{T}$ is confined to be less than $25 \mathrm{GeV}$. Note that the dimensionful numbers in the table are in $\mathrm{GeV}$ units. $\mathrm{L}$ and $\mathrm{H}$ denote low-mass and high-mass channels, respectively.

\begin{tabular}{|c|c|c|c|c|c|c|c|}
\hline \multirow{2}{*}{$\begin{array}{l}\text { Class } \\
\text { Subclass }\end{array}$} & \multicolumn{2}{|c|}{ Soft $1-\ell$ 1- $b$} & \multicolumn{2}{|c|}{ Soft $1-\ell 2-b$} & \multicolumn{2}{|c|}{ Soft $1-\ell$} & \multirow{2}{*}{$\begin{array}{c}\text { Soft } 2-\text { muon } \\
2-j\end{array}$} \\
\hline & $\mathrm{L}$ & $\mathrm{H}$ & $\mathrm{L}$ & $\mathrm{H}$ & $3-j$ & $5-j$ & \\
\hline Lepton & \multicolumn{6}{|c|}{$\begin{array}{c}N_{\ell}=1,10(6) \leq p_{T}^{\ell} \leq 25 \\
\ldots \\
\quad p_{T}^{\text {add } \cdot \ell}<7(6)\end{array}$} & $\begin{array}{c}2 \text { muons, } 6 \leq p_{T}^{\mu} \leq 25 \\
m_{\mu \mu}>15 \\
\left|m_{\mu \mu}-m_{Z}\right|>10\end{array}$ \\
\hline$N_{\text {jet }}$ & \multicolumn{2}{|c|}{$\geq 3$} & \multicolumn{2}{|c|}{$\geq 2$} & {$[3,4]$} & $\geq 5$ & $\geq 2$ \\
\hline$p_{T}^{j}$ & $>180,40,40$ & $>180,25,25$ & $>60$ & $<50$ & \multicolumn{2}{|c|}{$>180,25,25, \ldots$} & $>70,25,25 \ldots$ \\
\hline$N_{b-\operatorname{tag}}$ & \multicolumn{2}{|c|}{$\geq 1$, but not leading } & \multicolumn{2}{|c|}{2} & \multicolumn{2}{|c|}{$\ldots$} & 0 \\
\hline$E_{T}^{\text {miss }}>$ & 250 & 300 & 200 & 300 & 400 & 300 & 170 \\
\hline$m_{T}>$ & \multicolumn{2}{|c|}{100} & \multicolumn{2}{|c|}{$\cdots$} & \multicolumn{2}{|c|}{100} & 80 \\
\hline$E_{T}^{\mathrm{miss}} / m_{\mathrm{eff}}^{\mathrm{incl}}$ & \multicolumn{2}{|c|}{$>0.35$} & \multicolumn{2}{|c|}{$\cdots$} & \multicolumn{2}{|c|}{$>0.3$} & $\cdots$ \\
\hline$\Delta R_{\min }($ jet,$\ell)$ & \multicolumn{2}{|c|}{$>1.0$} & \multicolumn{2}{|c|}{$\cdots$} & $>1.0$ & $\cdots$ & $>1.0$ \\
\hline$\Delta \phi_{\min }$ & \multicolumn{2}{|c|}{$\cdots$} & \multicolumn{2}{|c|}{$>0.4$} & & & . \\
\hline$m_{C T}>$ & \multicolumn{2}{|c|}{$\cdots$} & 150 & 200 & & & . \\
\hline$H_{T 2}$ & \multicolumn{2}{|c|}{$\cdots$} & $<50$ & $\ldots$ & & & . \\
\hline$S_{\text {exp }}^{95}$ & $6.9_{-2.0}^{+3.0}$ & $6.3_{-1.1}^{+1.9}$ & $13.2_{-4.1}^{+5.9}$ & $5.3_{-1.4}^{+2.4}$ & $6.3_{-1.8}^{+2.7}$ & $10.0_{-3.0}^{+3.6}$ & $5.9_{-1.0}^{+2.1}$ \\
\hline
\end{tabular}

simplified model. Overall, we will find that the $E_{T}^{\text {miss }}$ cut is severe enough that in most cases the constraint on the ADM models will be much weaker than for the simplified model. Our discussion will also show, however, that better searches could easily be implemented by replacing the hard missing energy cut with a higher multiplicity of hard jets or leptons. Thus, it is desirable to compare the ADM model with the conventional SUSY models by performing similar LHC analyses with higher multiplicity (such as [38,39]). We postpone this study for future work.

\section{1-2-lepton +3 -6-jet + MET analysis}

The ATLAS 1-2-lepton + 3-6-jet + MET analysis with $20.3 \mathrm{fb}^{-1}$ at $\sqrt{s}=8 \mathrm{TeV}$ is summarized in Table III and in Table IV. This analysis effectively selects gluino and squark pair production events with one or two leptons from decays

TABLE IV. A summary of the ATLAS 1-2-lepton + 3-6-jet + MET analysis at $8 \mathrm{TeV}, 20.3 \mathrm{fb}^{-1}$. Part 2: Hard lepton events. The leading lepton $p_{T}$ must be higher than $25 \mathrm{GeV}$. The dimensionful numbers in the table are in GeV units.

\begin{tabular}{|c|c|c|c|c|c|c|c|c|c|c|c|c|}
\hline \multirow{3}{*}{$\begin{array}{l}\text { Class } \\
\text { Subclass } \\
\ell \text { type }\end{array}$} & \multicolumn{12}{|c|}{ Hard $1-\ell$} \\
\hline & \multicolumn{2}{|c|}{ Inclusive } & \multicolumn{2}{|c|}{ Binned } & \multicolumn{2}{|c|}{ Inclusive } & \multicolumn{2}{|c|}{ Binned } & \multicolumn{2}{|c|}{ Inclusive } & \multicolumn{2}{|c|}{ Binned } \\
\hline & $e$ & $\mu$ & $e$ & $\mu$ & $e$ & $\mu$ & $e$ & $\mu$ & $e$ & $\mu$ & $e$ & $\mu$ \\
\hline Lepton & \multicolumn{12}{|c|}{$N_{\ell}=1, p_{T}^{\ell}>25, p_{T}^{\text {add } \cdot \ell}<10$} \\
\hline$N_{\text {jet }}$ & \multicolumn{4}{|c|}{$\geq 3$} & \multicolumn{4}{|c|}{$\geq 5$} & \multicolumn{4}{|c|}{$\geq 6$} \\
\hline$p_{T}^{\mathrm{jet}}$ & \multicolumn{4}{|c|}{$>80,80,30$} & \multicolumn{4}{|c|}{$>80,50,40,40,40$} & \multicolumn{4}{|c|}{$>80,50,40,40,40,40$} \\
\hline$p_{T}^{\text {add.jets }}$ & \multicolumn{2}{|c|}{$\cdots$} & \multicolumn{2}{|c|}{$<40$} & & & \multicolumn{2}{|c|}{$<40$} & \multicolumn{2}{|c|}{$\cdots$} & \multicolumn{2}{|c|}{$<40$} \\
\hline$E_{T}^{\text {miss }}>$ & \multicolumn{2}{|c|}{500} & \multicolumn{2}{|c|}{300} & \multicolumn{4}{|c|}{300} & \multicolumn{2}{|c|}{350} & \multicolumn{2}{|c|}{250} \\
\hline$m_{T}>$ & \multicolumn{4}{|c|}{150} & \multicolumn{4}{|c|}{150} & \multicolumn{4}{|c|}{150} \\
\hline$E_{T}^{\text {miss }} / m_{\mathrm{eff}}^{\text {excl. }}$ & \multicolumn{4}{|c|}{$>0.3$} & \multicolumn{8}{|c|}{$\cdots$} \\
\hline$m_{\mathrm{eff}}^{\mathrm{incl}}$ & \multicolumn{2}{|c|}{1400} & \multicolumn{2}{|c|}{800} & \multicolumn{2}{|c|}{1400} & \multicolumn{2}{|c|}{800} & & & & \\
\hline $\begin{array}{l}S_{\text {exp }}^{95} \\
\text { Error }\end{array}$ & $\begin{array}{c}5.7 \\
+2.2 \\
-1.5\end{array}$ & $\begin{array}{c}5.1 \\
+2.0 \\
-1.5\end{array}$ & $\begin{array}{l}20.2 \\
-4.8 \\
+8.3\end{array}$ & $\begin{array}{r}15.6 \\
-3.8 \\
+5.8\end{array}$ & $\begin{array}{c}5.4 \\
-1.5 \\
+2.3\end{array}$ & $\begin{array}{c}4.7 \\
-1.2 \\
+1.9\end{array}$ & $\begin{array}{r}12.6 \\
-2.7 \\
+3.2\end{array}$ & $\begin{array}{c}7.6 \\
-2.4 \\
+2.8\end{array}$ & $\begin{array}{c}4.4 \\
+1.9 \\
-0.8\end{array}$ & $\begin{array}{c}4.1 \\
+1.3 \\
-1.1\end{array}$ & $\begin{array}{l}7.8 \\
+3.1 \\
-2.4\end{array}$ & $\begin{array}{c}7.1 \\
+3.4 \\
-1.4\end{array}$ \\
\hline
\end{tabular}


of charginos or sleptons. The analysis is divided into soft and hard lepton channels. Signal leptons with $p_{T}<25 \mathrm{GeV}$ are regarded as soft and in turn have seven classes: soft single lepton $1 b$-jet low-mass/high-mass, soft single lepton $2 b$-jets low-mass/high-mass, soft single lepton 3-jet/5-jet and soft dimuon channels. Hard lepton channels have three classes: 3 -jet, 5-jet and 6-jet channels, with each class having inclusive/binned channels and electron/muon subchannels according to the lepton identity. Thus there are 12 channels in total for the hard lepton case. We summarize the requirements and the observed 95\% C.L. limit of this analysis from the ATLAS experiment in Tables III, IV.

For the 1-2-lepton analysis, we compare the $q \ell d^{c}$ model with the simplified models by varying the relative ratio between colored SUSY particle masses and the LOSP mass. To this end, we use two simplified models as shown in Fig. 8, which are referred to as "one-step" simplified models in the ATLAS analysis [40]. The first model, shown in Fig. 8(a), which we call "Simlg," has the gluino $\tilde{g}$, the lightest chargino $\chi_{1}^{ \pm}$and the lightest neutralino $\chi_{1}^{0}$. Only a pair of gluinos are assumed to be produced, with the gluino decaying via $\tilde{g} \rightarrow q \bar{q}^{\prime} \chi_{1}^{ \pm} \rightarrow q \bar{q}^{\prime} W^{(*)} \chi_{1}^{0}$ with $100 \%$ branching, where $q$ and $q^{\prime}$ are quarks with different isospin and $W^{(*)}$ is the on-shell (off-shell) $W$ boson, depending on the mass gap between $\chi_{1}^{ \pm}$and $\chi_{1}^{0}$. The second model shown in Fig. 8(b), which we call "Simlq," has the left-handed squark $\tilde{q}_{L}$, the lightest chargino $\chi_{1}^{ \pm}$and the lightest neutralino $\chi_{1}^{0}$. Note that only left-handed squarks are involved since $\chi_{1}^{ \pm}$and $\chi_{1}^{0}$ are assumed to be mostly Winolike. Now the production is only through squark pairs with the squark decaying through $\tilde{q}_{L} \rightarrow q^{\prime} \chi_{1}^{ \pm} \rightarrow q^{\prime} W^{(*)} \chi_{1}^{0}$. For simplicity, ${ }^{3}$ we fix the ratio among the colored superparticle $\left(\tilde{g} / \tilde{q}_{L}\right), \chi_{1}^{ \pm}$and $\chi_{1}^{0}$,

$$
m_{\chi_{1}^{ \pm}}=\frac{m_{\tilde{g}(\tilde{q})}-m_{\chi_{1}^{0}}}{2}
$$

Similarly to the 0 lepton analysis, we find $E_{T}^{\text {miss }}$ to be a key variable in distinguishing the ADM model from the simplified models, though the $p_{T}$ cut on both the hardest lepton and jet will play an important role. Note, however, that the $E_{T}^{\text {miss }}$ cut here is stronger than in the 0-lepton analysis in order to filter the SM $W$ and top-quark events. For some soft channels, $b$ tagging is employed, and thus the $b$-tagging efficiency affects the event acceptance. In the ATLAS analysis, different $b$-tagging efficiency has been applied by adjusting a $b$-tagging parameter for different channels. However, in our analysis, we simply rely on the detector simulator that we use; since the efficiency difference is at the $\sim 10 \%$ level and cross-section differences between two adjacent scan points are much higher, our

\footnotetext{
${ }^{3}$ Admittedly, this choice is far from general. We simply follow the ATLAS analysis here for comparison with the $q \ell d^{c}$ model in the $m_{\tilde{g}}\left(m_{\tilde{q}}\right)-m_{\chi_{1}^{0}}$ scan.
}

results will not be significantly changed because of the $b$-tagging method.

\section{B. Event generation}

We use MadGraph5 v1.5.8 for the matrix-element (ME) event generation [41]. The generated events are reweighted to match the next-to-leading-order (NLO) cross section. We employ Prospino 2.1 to obtain the cross section of gluino and squark pair production at NLO $[42,43]$.

Since the processes under consideration consist of cascades of multiple decay chains through on-shell states with very narrow decay widths, it is desirable to divide a single process into one 2-to-2 process and multiple decay subprocesses for each on-shell particle in the process, to generate events for them separately, and to merge all of the subparts into a single process by doing the appropriate Lorentz transformation and color flow matching. ${ }^{4} \mathrm{We}$ created a utility called EVCHAIN for doing the job automatically [44]. A detailed description of EVCHAIN is presented in Appendix D. We use PYTHIA6 for the $q \ell d^{c}$ model and PYTHIA8 for the $u^{c} d^{c} d^{c}$ model for a parton shower (PS) and hadronization ${ }^{5}[45,46]$. We generate only leading order SUSY events without employing matrix-element or parton shower matching, assuming the LO parton-showered distribution scaled with the NLO $K$ factor approximates the true distribution well for large $\sqrt{s}$ for typical gluino/squark production. Because we do not use a matched sample for the signal events, our result should be interpreted with care in the compressed mass spectrum where the $p_{T}$ of additional QCD jets can be comparable to the $p_{T}$ of jets from superparticle decay, significantly changing the $p_{T}$ distribution of the leading jets. Such points need further focused study with appropriate matching. For detector simulation, we modify PGS4 to enable anti- $k_{T}$ jet reconstruction, and we rely on the $b$-tagging efficiency implemented in PGS4 [47].

For the 0-lepton analysis, we scan mass parameters in the gluino-common-squark mass plane by fixing the neutralino mass $m_{\chi_{1}^{0}}$. For the ADM model, we fix the mass of the $\mathrm{ADM}$ to be $10 \mathrm{GeV}$ (a well-motivated value), and we consider four different cases: a squark LOSP (with the neutralino decoupled), and a neutralino LOSP with $m_{\chi_{1}^{0}}=100,300$ and $500 \mathrm{GeV}$. The simplified model SimO is scanned in the same $\left(m_{\tilde{q}}, m_{\tilde{q}}\right)$ mass plane with the neutralino mass $m_{\chi_{1}^{0}}=10,100,300$ and $500 \mathrm{GeV}$, where $10 \mathrm{GeV}$ is chosen for comparison with the ADM model with a squark LOSP. The gluino and squark mass parameters are scanned by generating 10,000 events for each parameter, from 100 to $3000 \mathrm{GeV}$ with $100 \mathrm{GeV}$ spacing. For a squark LOSP, we additionally impose the condition $m_{\tilde{g}}>m_{\tilde{q}}$. For high cross-section regions where

\footnotetext{
${ }^{4}$ In this paper, we do not consider spin correlation.

${ }^{5}$ PYTHIA 6 does not support the color-triplet vertex $\left(\epsilon_{i j k}\right)$ as an acceptable color flow structure.
} 


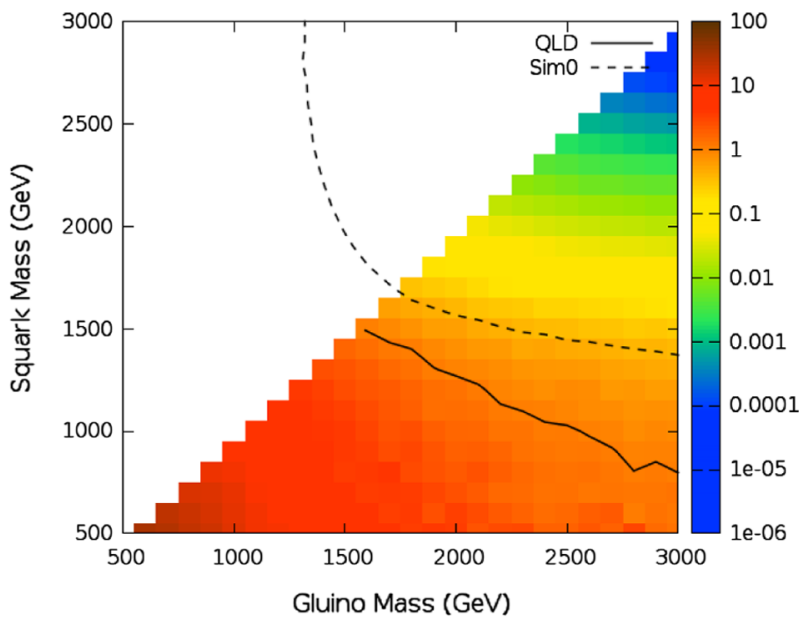

(a) 0 lepton+ 2-6 jets $+\mathrm{MET}$

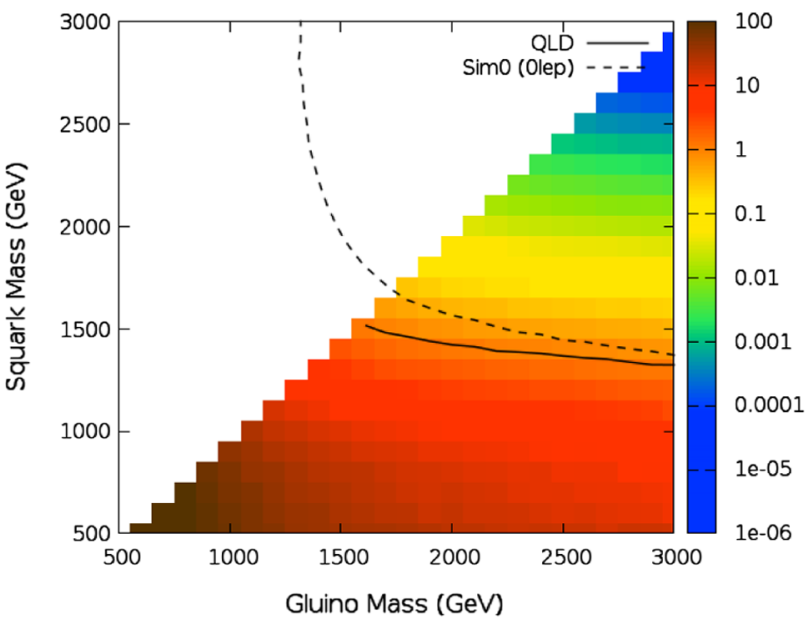

(b) 1-2 lepton + 3-6 jets + MET

FIG. 11 (color online). (a) ATLAS 0-lepton + 2-6-jet + MET/(b) 1-2-lepton + 3-6-jet + MET analyses for the $q \ell d^{c}$ model with a squark LOSP (solid line) in the $\left(m_{\tilde{g}}, m_{\tilde{q}}\right)$ plane compared to the simplified model Sim0 of Fig. 7 (dashed line). Here the constraint on the simplified model SimO is taken from the result of the 0-lepton analysis for both figures. The neutralino mass $m_{\chi_{1}^{0}}$ for $\operatorname{Sim} 0$ is $10 \mathrm{GeV}$ for comparison with the ADM mass in the $q \ell d^{c}$ model.

$m_{\tilde{g}}$ or $m_{\tilde{q}}$ is below $1000 \mathrm{GeV}$, we scale the number of events as needed to reduce statistical errors.

For the 1-2-lepton analysis, in which only the $q \ell d^{c}$ model is relevant, we additionally scan the mass parameters in the planes of $\left(m_{\tilde{g}}, m_{\chi_{1}^{0}}\right)$ and $\left(m_{\tilde{q}}, m_{\chi_{1}^{0}}\right)$ with decoupled squarks and gluinos, respectively. The $\left(m_{\tilde{g}}, m_{\chi_{1}^{0}}\right)$ scan is compared to the simplified model Simlg, and the $\left(m_{\tilde{q}}, m_{\chi_{1}^{0}}\right)$ scan is compared to the simplified model Simlq; thus, we generate events for those simplified models in the same scanning. Due to reduced experimental sensitivity, the scan region is confined to $1500 \mathrm{GeV}$ for $m_{\tilde{g}}$, to $1300 \mathrm{GeV}$ for $m_{\tilde{q}}$, and to $1000 \mathrm{GeV}$ for $m_{\chi_{1}^{0}}$. We reduce the grid spacing to $50 \mathrm{GeV}$ for this scan. We also show the 1-2-lepton constraint for the $q \ell d^{c}$ model in the $\left(m_{\tilde{g}}, m_{\tilde{q}}\right)$ plane, but we compare the result only with the 0-lepton analysis constraint for the simplified model Sim0. Again, the ADM mass is fixed to be $10 \mathrm{GeV}$.

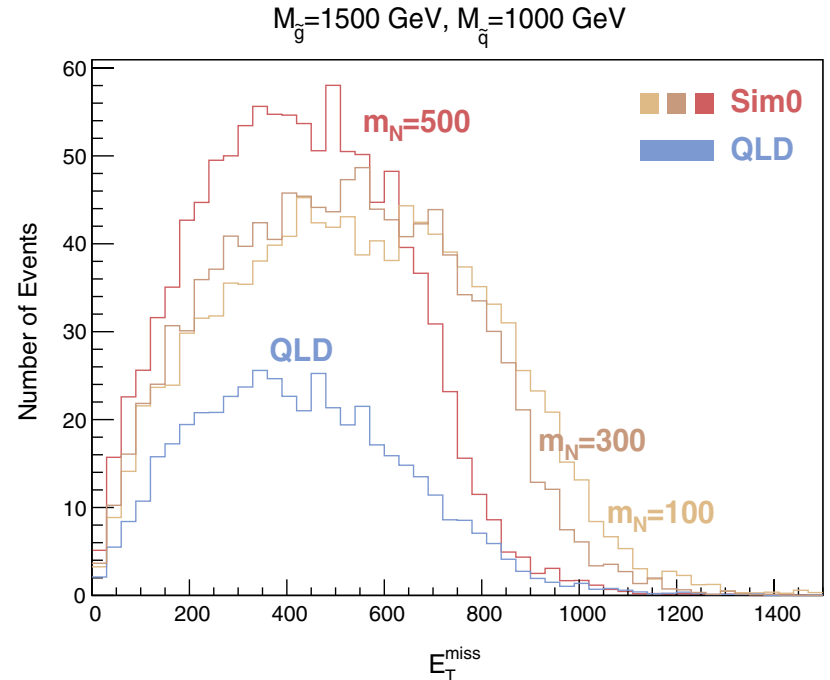

(a) $E_{T}^{\text {miss }}$

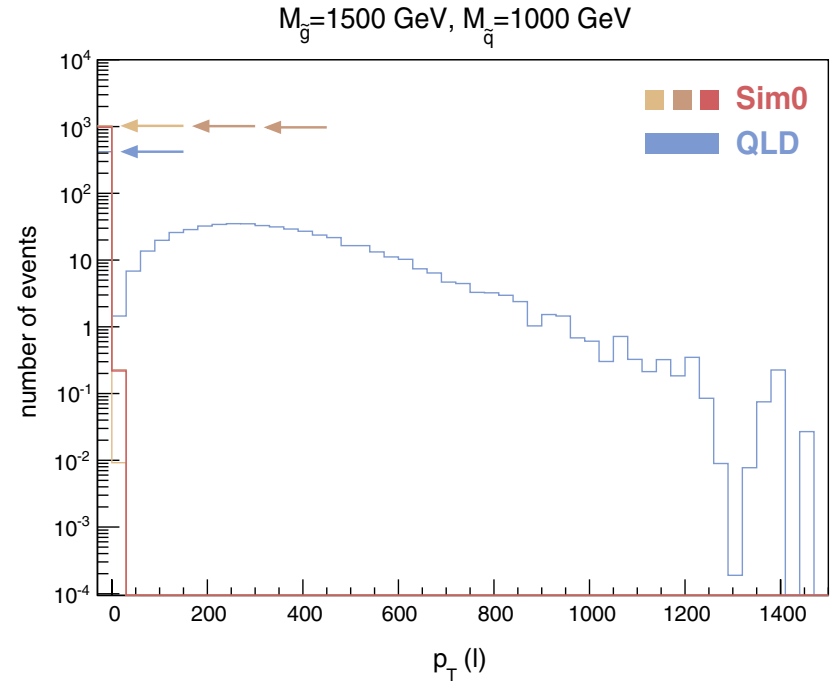

(b) Hardest lepton $p_{T}$

FIG. 12 (color online). (a) The missing transverse energy (MET) distribution and (b) the $p_{T}$ distribution of the hardest lepton of the $X q \ell d^{c}$ model with a squark LOSP (blue histogram) and simplified models Sim0 (red histograms). For the simplified models, we show three different neutralino masses $m_{\chi_{1}^{0}}=100,300$ and $500 \mathrm{GeV}$. For the lepton $p_{T}$, the first bin shows the number of events that pass the lepton veto cut of the 0-lepton analysis. We indicate the first bin using arrows in the right panel. The color scheme for the neutralino mass is the same for both graphs. 


\section{Results}

We discuss our results for the $q \ell d^{c}$ model, followed by those for the $u^{c} d^{c} d^{c}$ model. For the former model, we apply both the 0-lepton and 1-2-lepton analyses, while for the latter we apply the 0-lepton analysis only. In each case, we consider a squark LOSP decay into the ADM sector first (which is topologically most similar to the simplified model for comparison), before constraining a neutralino LOSP decay into the ADM sector.

$$
\text { 1. } W=X \boldsymbol{q} \ell \boldsymbol{d}^{c}
$$

Squark LOSP.-We first present the squark LOSP case of the q $\ell d^{c}$ model via the diagrams of Fig. 9. For the sake of simplification, we assume the first two-generation squarks are nearly degenerate in mass but have a large enough mass splitting that the heavier squarks decay promptly to very soft (undetectable) jets and leptons and a lighter squark until the lightest squark is reached at the bottom of the cascade. We implement this by putting a $5 \mathrm{GeV}$ mass splitting between the lightest squark and the others as a maximal allowed value for very soft jets and leptons. ${ }^{6}$ The LOSP squark finally decays to the ADM with a quark and a lepton or neutrino. Hence, additional jets and leptons appear in the event, but the missing energy is reduced. Note that even if there is no splitting, the other squarks will likely decay promptly to the ADM through different ADM operators, and the phenomenology is the same in such cases.

The results of the ATLAS 0-lepton + 2-6-jet + MET and the ATLAS 1-2-lepton + 3-6-jet + MET analyses at $\sqrt{s}=8 \mathrm{TeV}$ with a luminosity of $20.3 \mathrm{fb}^{-1}$ for the squark LOSP $q \ell d^{c}$ case are shown in Fig. 11. The color level shows the maximum of $S_{i} / S_{\exp , i}^{95}$ for all channels $i$, where $S_{i}$ is the number of events for the channel from our event generation at a given point, and $S_{i} / S_{\mathrm{exp}, i}^{95}$ from the analysis given in Table II (for Fig. 11(a)) and Tables III and IV (for Fig. 11(b)). Thus, the contour at 1 (shown as the dashed or solid lines) can be interpreted roughly as the 95\% C.L. exclusion. ${ }^{7}$ In the plots, we show the simplified model $\operatorname{Sim} 0$ exclusion contour by performing the same 0-lepton analysis. The neutralino mass for the $\operatorname{Sim} 0$ model is $10 \mathrm{GeV}$.

The 0-lepton analysis result shows that the constraint is weaker for the $q \ell d^{c}$ model, while the 1-2-lepton analysis constraint for the $q \ell d^{c}$ model is similarly matched with the simplified model Sim0 0-lepton analysis constraint. The reason why the constraint from the 0-lepton analysis on the $q \ell d^{c}$ model is weaker is simply because half of the LOSP squarks decay into a charged lepton, which is vetoed in the analysis.

\footnotetext{
${ }^{6}$ However, this rather large splitting can cause additional flavor violation in low energy observables.

${ }^{7} \mathrm{~A}$ correct interpretation of the confidence level by combining such multiple nonexclusive channels must be taken with care, and it is beyond the scope of this paper.
}

To see this, we show the MET distribution and the $p_{T}$ distribution of the hardest lepton in Fig. 12 at a mass parameter point $\left(m_{\tilde{g}}, m_{\tilde{q}}\right)=(1500 \mathrm{GeV}, 1000 \mathrm{GeV})$. The MET distribution in Fig. 12(a) is obtained after applying signal object identification/isolation, the lepton veto, and the two hardest jet $p_{T}$ cuts: $p_{T}\left(j_{1}\right)>130 \mathrm{GeV}$, $p_{T}\left(j_{2}\right)>60 \mathrm{GeV}$, from the 0-lepton analysis. The $p_{T}$ distribution of the hardest lepton in Fig. 12(b) is obtained after applying the same cuts except the lepton veto cut, instead applying the MET cut: $E_{T}^{\text {miss }}>160 \mathrm{GeV}$. One can easily see that the MET distribution in Fig. 12(a) is not very different for the simplified model $\operatorname{Sim} 0$ than for the $q \ell d^{c}$ model, though the rate is different due to the lepton veto as one can see in the lepton $p_{T}$ distribution in Fig. 12(b): The simplified model SimO has $100 \%$ no-lepton events, while the $q l d^{c}$ model has $45 \%$ no-lepton events.

Neutralino LOSP.-Next, we present the constraints for the neutralino LOSP case of the $q \ell d^{c}$ model via the diagrams of Fig. 10. In this case, we do not have to assume a splitting between squarks since squarks decay promptly into the neutralino. The $\left(m_{\tilde{g}}, m_{\tilde{q}}\right)$ scan results of the ATLAS 0-lepton and 1-2-lepton analyses are shown in Fig. 13 for three different neutralino mass choices: $m_{\chi_{1}^{0}}=100,300$ and $500 \mathrm{GeV}$. Again, we compare the result of the qld model with the O-lepton analysis of the simplified model Sim0 with the same neutralino mass parameters. The contours of $\operatorname{maximum}_{i}\left(S_{i} / S_{\mathrm{exp}, i}^{95}\right)=1$ for qedc and $\operatorname{Sim} 0$ are drawn as solid and dashed curves, respectively.

The constraints for the neutralino LOSP $q \ell d^{c}$ model are generically weaker than the simplified model $\operatorname{Sim} 0$ for small $m_{\chi_{1}^{0}}(100$ and $300 \mathrm{GeV})$ but reveal a more complicated behavior in the $m_{\chi_{1}^{0}}=500 \mathrm{GeV}$ case. Several factors contribute to these results. One obvious factor that tends to give weaker constraints on the ADM model in the 0-lepton analysis is the branching fraction to charged leptons, which we have already seen in the squark LOSP case. More importantly, the missing energy of the neutralino is reduced as it decays to two additional jets plus a lepton. This feature is transparently comparable to the simplified model $\operatorname{Sim} 0$ since both models share the same event topology before the neutralino decay. On the other hand, as the neutralino mass is set heavier, the energy of the jets from th gluino/squark decay into the neutralino becomes smaller as the mass difference shrinks. Therefore, the experimental sensitivity to the simplified model $\operatorname{Sim} 0$ (and ordinary $R$-parity conserving MSSM scenarios, generically) is reduced for a heavier neutralino mass, while the ADM models are subject to more severe constraints since a massive neutralino is able to "store" and transfer energy to the ADM particle. Therefore, for large neutralino mass, the ADM model can actually become substantially more constrained than the simplified model. 


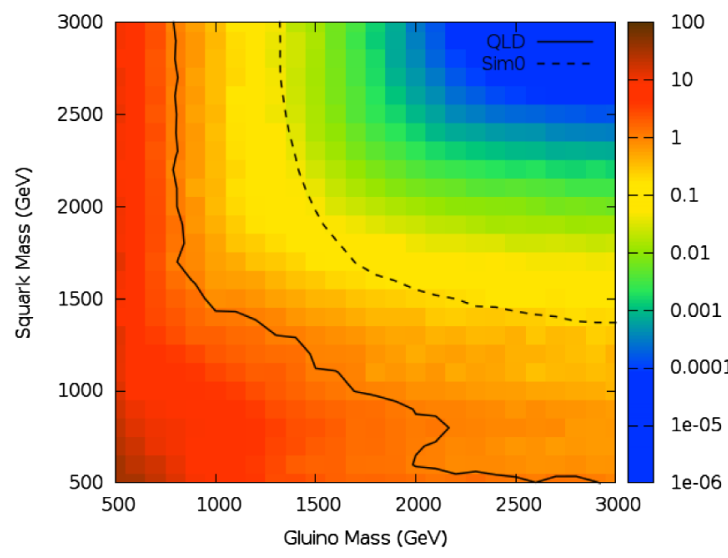

(a) 0 lepton analysis for $m_{\chi_{1}^{0}}=100 \mathrm{GeV}$

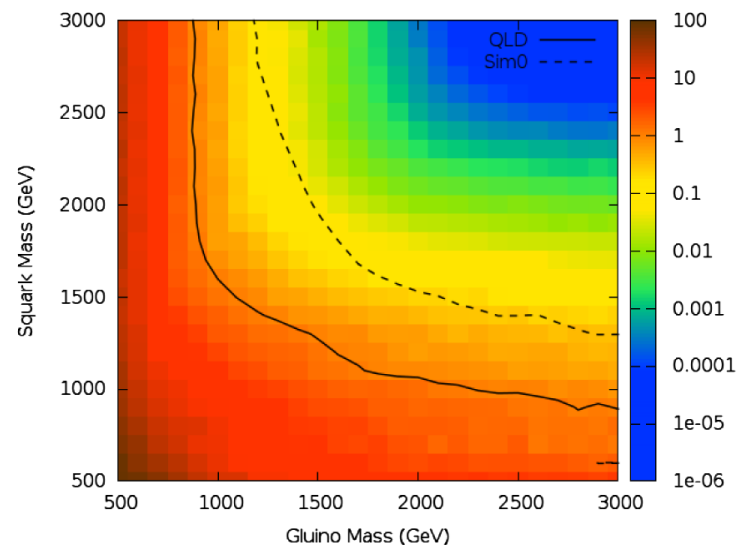

(c) 0 lepton analysis for $m_{\chi_{1}^{0}}=300 \mathrm{GeV}$

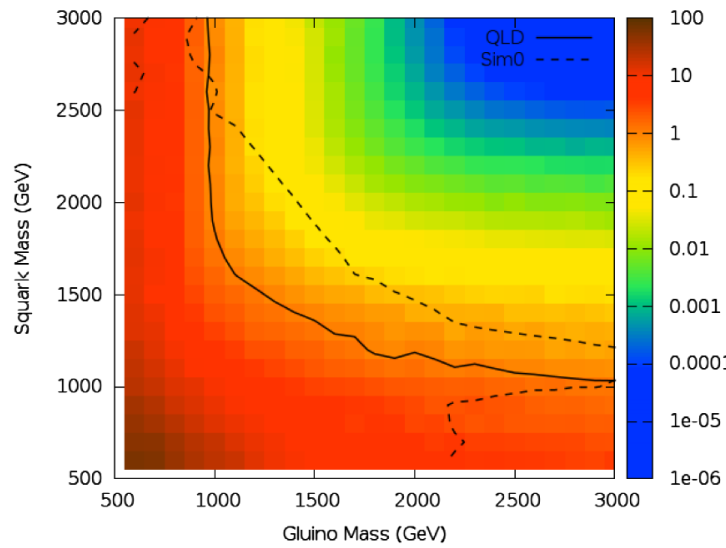

(e) 0 lepton analysis for $m_{\chi_{1}^{0}}=500 \mathrm{GeV}$

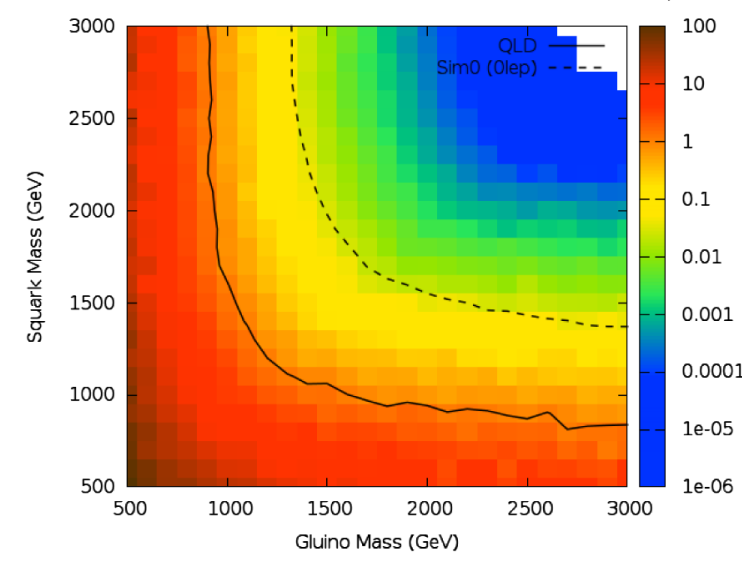

(b) 1-2 lepton analysis for $m_{\chi_{1}^{0}}=100 \mathrm{GeV}$

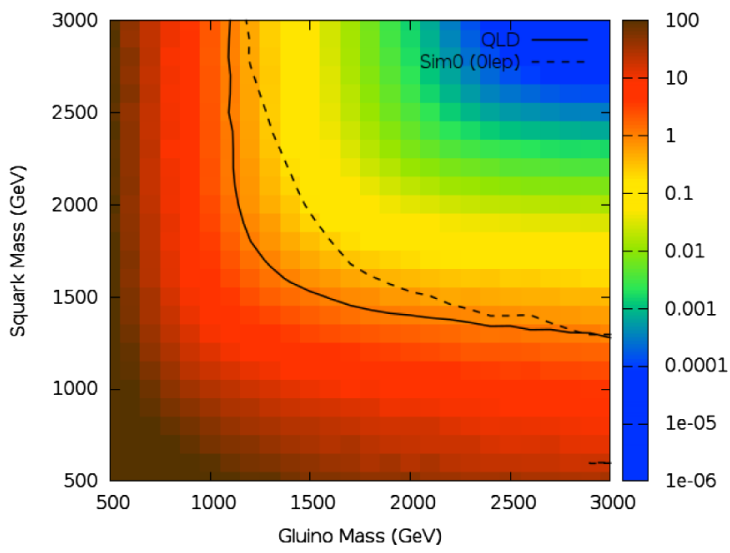

(d) 1-2 lepton analysis for $m_{\chi_{1}^{0}}=300 \mathrm{GeV}$

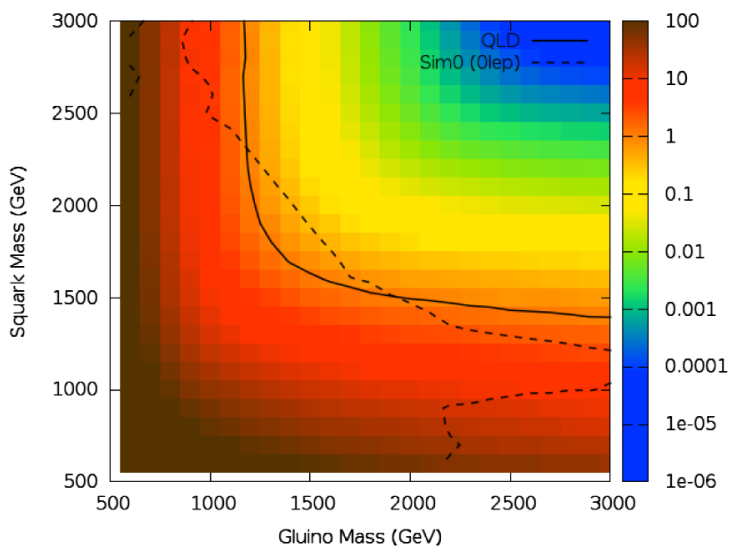

(f) 1-2 lepton analysis for $m_{\chi_{1}^{0}}=500 \mathrm{GeV}$

FIG. 13 (color online). Constraint from ATLAS 0-lepton [left panels (a), (c), (e)] and 1-2-lepton [right panels (b), (d), (f)] analyses on the $q \ell d^{c}$ model with a neutralino LOSP (solid line) in the $\left(m_{\tilde{g}}, m_{\tilde{q}}\right)$ plane, compared to the simplified model SimO (dashed line). Here the constraint on the $\mathrm{Sim} O$ model of Fig. 7 is taken from the 0-lepton analysis result for both the left- and right-hand plots. The $X q \ell d^{c}$ model with a neutralino LOSP decays through Fig. 10, with $m_{\chi_{1}^{0}}=100,300$ and $500 \mathrm{GeV}$.

In Fig. 14, we compare the MET distribution and the hardest lepton $p_{T}$ distribution of the neutralino LOSP $q \ell d^{c}$ model and the simplified model Sim0 for $m_{\tilde{g}}=m_{\tilde{q}}=$ $1000 \mathrm{GeV}$ and $m_{\chi_{1}^{0}}=100,300$ and $500 \mathrm{GeV}$. Here, we use the same cuts as in Fig. 12. Note that $E_{T}^{\text {miss }}$ is distinctively smaller for the $q \ell d^{c}$ case. For the lepton
$p_{T}$ distribution, the first bin implies events that pass the lepton veto cut. Note the significant difference among different $m_{\chi_{1}^{0}}$ 's in the lepton veto and $p_{T}$ for the $q \ell d^{c}$ models. The acceptance of the lepton veto is $78.3 \%, 47.8 \%$ and $41.9 \%$ for 100,300 and $500 \mathrm{GeV}$ neutralino, respectively, for the $q \ell d^{c}$ model, while the acceptance of the 


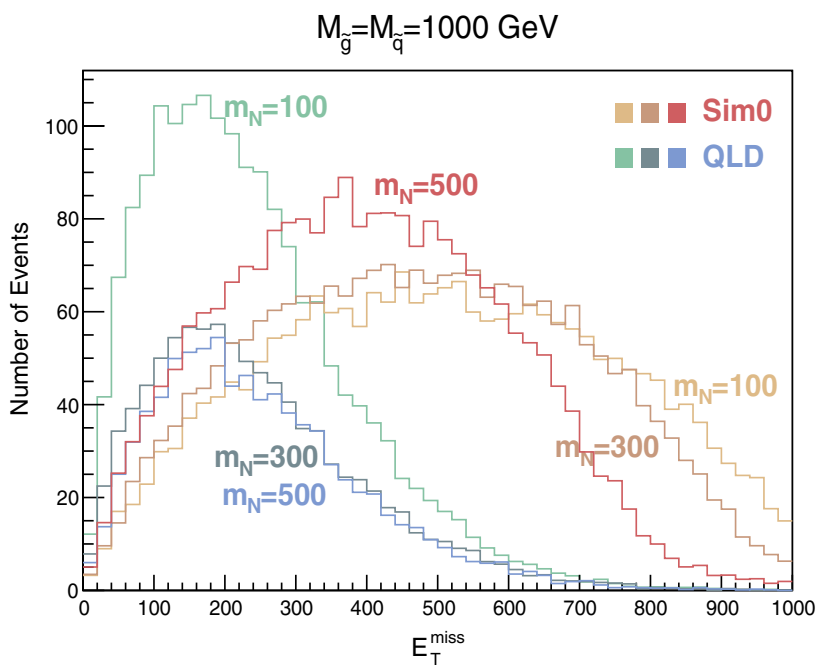

(a) $E_{T}^{\text {miss }}$

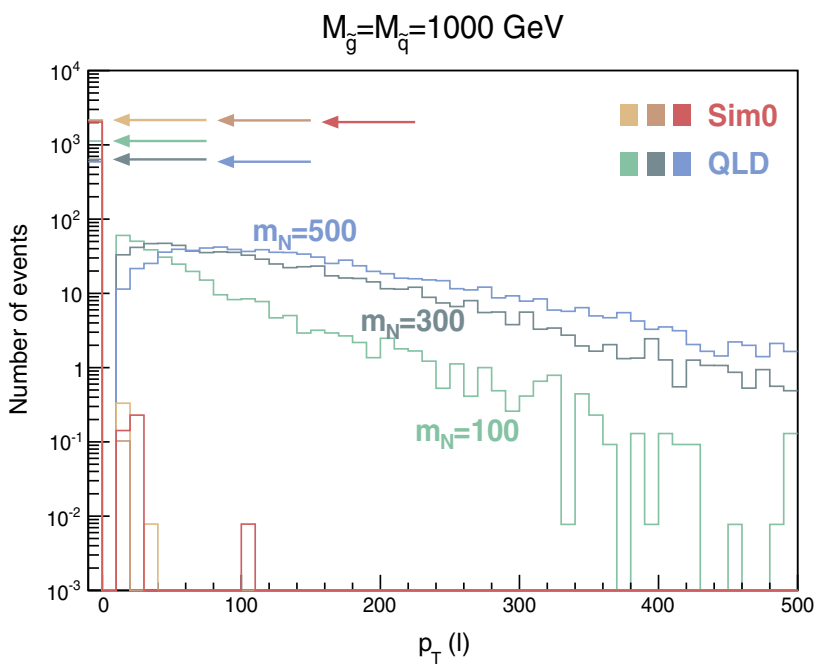

(b)Hardest lepton $p_{T}$

FIG. 14 (color online). (a) The MET and (b) transverse momentum $p_{T}$ of the hardest lepton distributions in the $q \ell d^{c}$ model (blue histograms) and simplified model Sim0 (red histograms) for $m_{\tilde{g}}=m_{\tilde{q}}=1000 \mathrm{GeV}$ and $m_{\chi_{1}^{0}}=100,300$ and $500 \mathrm{GeV}$. In the right panel, the first bin shows the number of events that pass the lepton veto cut of the 0-lepton analysis. We indicate the first bin using arrows in the right panel. The color scheme for the neutralino mass is the same for both graphs.

lepton veto is nearly $100 \%$ for the simplified model Sim0. This implies that leptons from light $\chi_{1}^{0}$ decay often fail the lepton veto cut $\left(p_{T}^{\ell}<10 \mathrm{GeV}\right.$ in this case).

Lastly, in Fig. 15, we compare the constraints from the ATLAS 1-2-lepton analysis for the neutralino LOSP $q \ell d^{c}$ model and the simplified model Simlg (Simlq) in the gluino (squark)-neutralino plane. The constraints have completely different behaviors for each model from the same analysis because the decay of a massive neutralino results in high $p_{T}$ and MET in $q \ell d^{c}$ while a smaller gap between the neutralino and the gluino (squark) tends to give softer jets and MET in the simplified models. This feature is

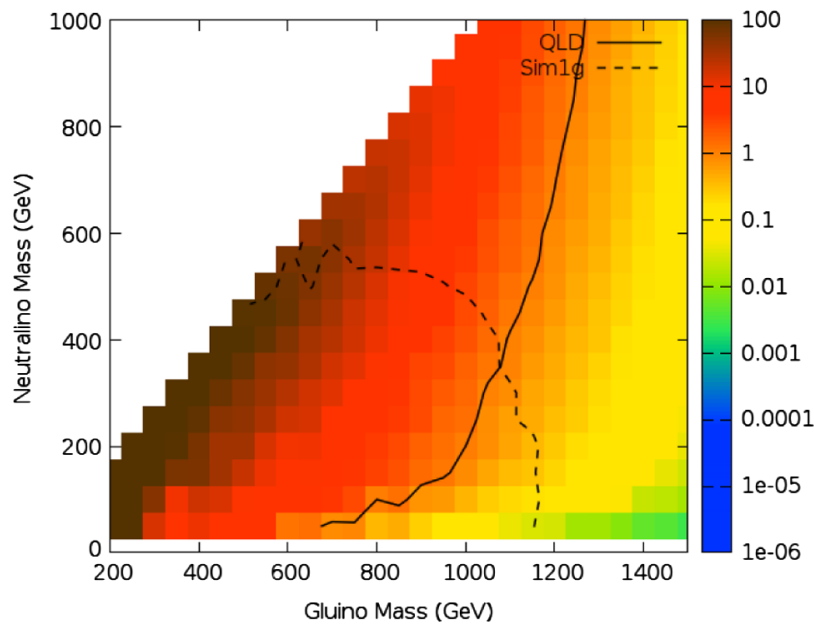

(a) gluino-neutralino scan illustrated clearly in the MET and the hardest jet $p_{T}$ distributions, shown in Fig. 16 for two benchmark points: (A) $m_{\tilde{g}}=1000 \mathrm{GeV}$ and $m_{\chi_{1}^{0}}=800 \mathrm{GeV}$, (B) $m_{\tilde{g}}=$ $1000 \mathrm{GeV}$ and $m_{\chi_{1}^{0}}=100 \mathrm{GeV}$. To obtain Fig. 16, we applied the $p_{T}$ cut of the "Soft $1-\ell$ " class (in Table III) to the hardest three jets for soft lepton events, and we applied the $p_{T}$ cut of the "Hard $1-\ell 3$ jet" class (in Table III) to the hardest three jets for hard lepton events. One can easily see that the MET and $p_{T}\left(j_{1}\right)$ of the $q \ell d^{c}$ model (blue) are higher for benchmark point A (above), but the MET and $p_{T}\left(j_{1}\right)$ of the simplified model (red) are higher for point B (below).

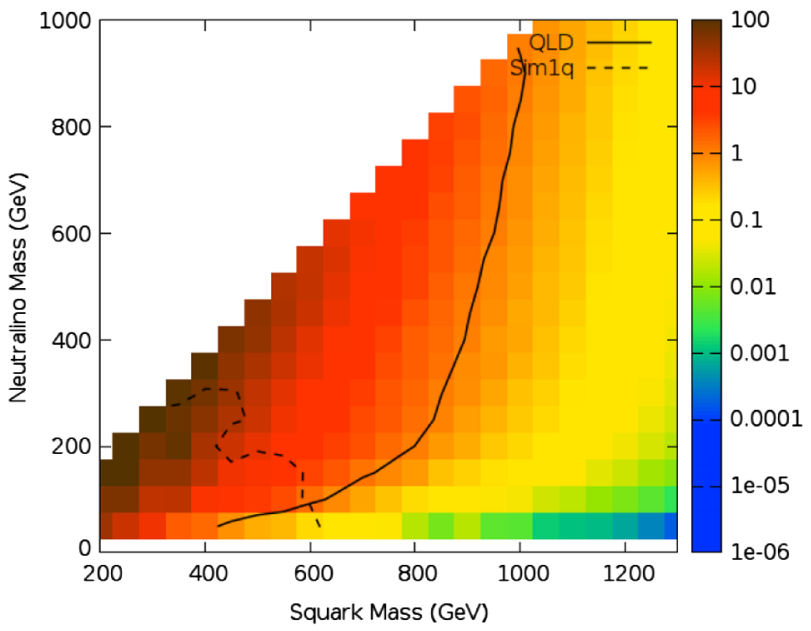

(b) squark-neutralino scan

FIG. 15 (color online). ATLAS 1-2-lepton + 3-6-jet + MET SUSY search analysis for the $q \ell d^{c}$ model. Here the constraints on the MSSM simplified model of Fig. 8 are compared against the $q \ell d^{c}$ model with a neutralino LOSP decaying through Fig. 10. The squarks have been decoupled in the left panel (a), while in the right panel (b) the gluinos have been decoupled. 

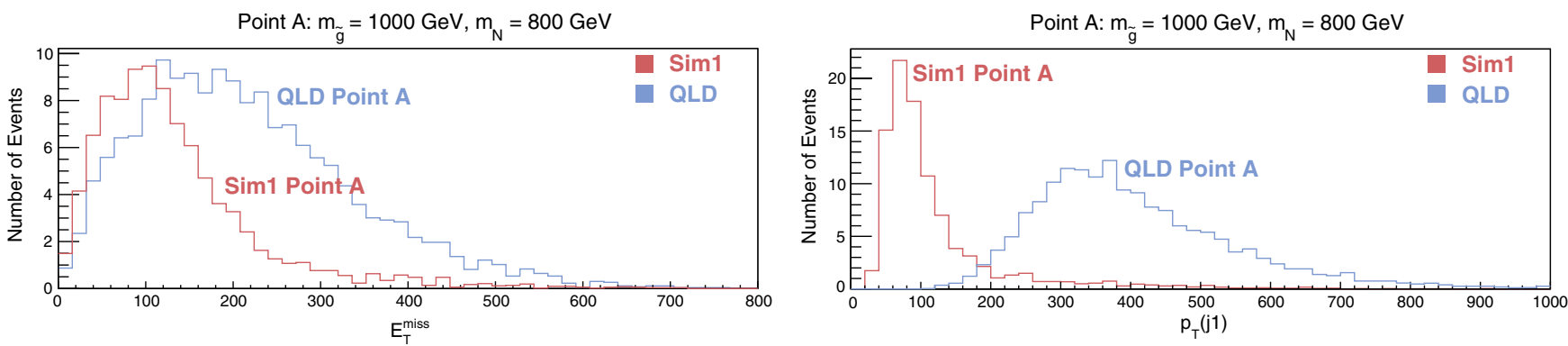

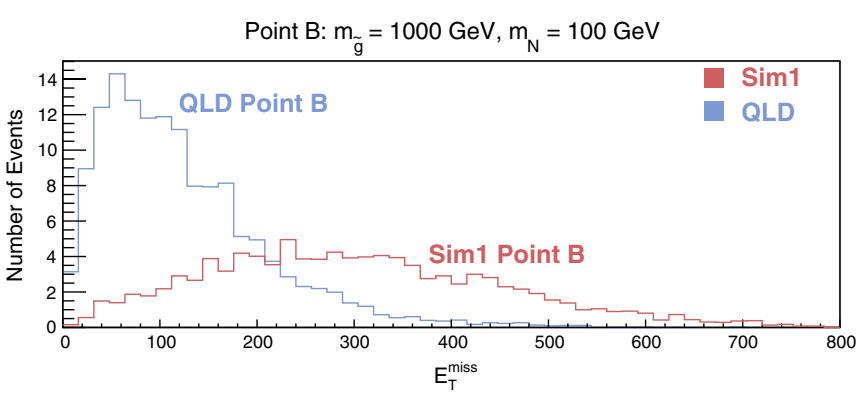

(a) $E_{T}^{\text {miss }}$

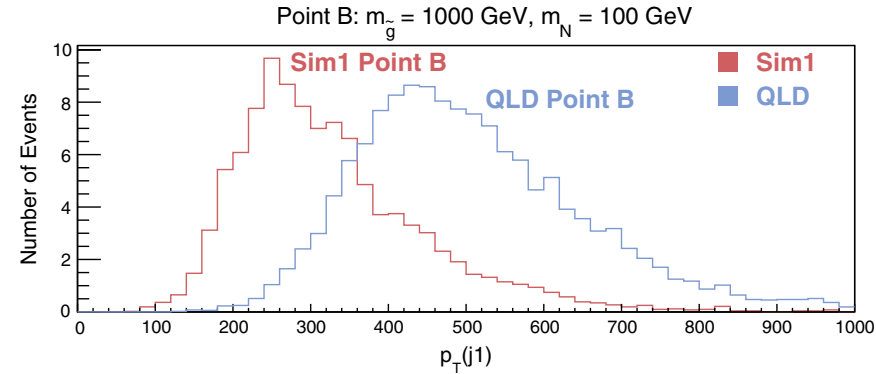

(b) $p_{T}(j 1)$

FIG. 16 (color online). (a) The missing transverse energy distribution and (b) hardest jet $p_{T}$ distribution of the neutralino LOSP $q \ell d^{c}$ model with the squark decoupled and the simplified model Simlg in the 1-2-lepton analysis. Here, we chose two points of mass parameter: (A) $m_{\tilde{g}}=1000 \mathrm{GeV}, m_{\chi_{1}^{0}}=800 \mathrm{GeVand}$ (B) $m_{\tilde{g}}=1000 \mathrm{GeV}, m_{\chi_{1}^{0}}=100 \mathrm{GeV}$.

We commment on a possibility of observing the states in the UV completion from which the effective ADM operators are generated. In principle, these states, $Q, L$ and $D$, can be directly produced at the collider. When these states decay to the DM, $Q, D \rightarrow X+q$ and $L \rightarrow X+\ell$, the signatures look similar to squark or stop signatures of the jet or top quark plus missing energy, or slepton and sneutrino decays to a lepton plus missing energy. On the other hand, these states may have more exotic decays, for example, to a lepton and a jet, or to flavor-violating pairs of quarks such as a top and a light flavor jet. For example, we may have

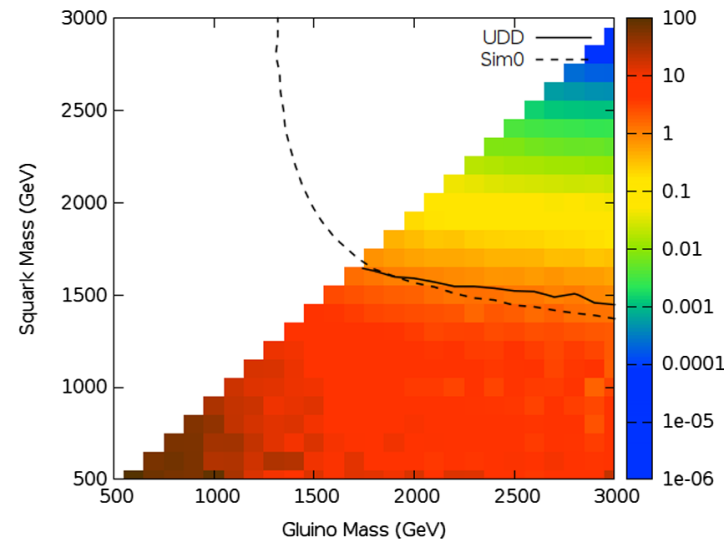

FIG. 17 (color online). ATLAS 0-lepton + 2-6-jet + MET analysis for the $u^{c} d^{c} d^{c}$ model with a squark LOSP (solid curve) and the simplified model Sim0 (dashed curve). The solid curve ends for the $u^{c} d^{c} d^{c}$ model when the squark is no longer the LOSP.

$$
D \rightarrow u \ell^{-} \quad Q_{u, d} \rightarrow\left(\ell^{+}, \nu\right) d,
$$

leading to the possibility of spectacular decay modes at the LHC, which are similar in spirit to leptoquark searches at the LHC. The study of such signatures could give rise to interesting further constraints on ADM models.

$$
\text { 2. } W=X u^{c} d^{c} d^{c}
$$

Squark LOSP.-Now we carry out the ATLAS 0-lepton analysis for the $u^{c} d^{c} d^{c}$ models and compare the result with the simplified model Sim0. First, we consider the squark LOSP case of the $u^{c} d^{c} d^{c}$ models. As in the qed model case, we assume that squarks have a large enough mass splitting for prompt decay to the lightest squark in the $u^{c} d^{c} d^{c}$ model, which is again implemented by a $5 \mathrm{GeV}$ splitting between the lightest and other squarks. The relevant processes at the LHC are given in Fig. 9 with a lepton or neutrino replaced by a jet in the lightest squark decay. The ADM mass is $10 \mathrm{GeV}$ here, and for Sim0, we set $m_{\chi_{1}^{0}}=10 \mathrm{GeV}$ for a fair comparison.

Figure 17 shows the constraints from the 0-lepton analysis at $\sqrt{s}=8 \mathrm{TeV}, 20.3 \mathrm{fb}^{-1}$ for the squark LOSP $u^{c} d^{c} d^{c}$ and the Sim0 model. The color level represents $\max _{i}\left(S_{i} / S_{\mathrm{exp}, i}^{95}\right)$ for the $u^{c} d^{c} d^{c}$ model, similarly to the $q \ell d^{c}$ case, and thus the contour at 1 corresponds to $95 \%$ C.L., roughly. Interestingly, the constraints for both the $u^{c} d^{c} d^{c}$ model (solid line) and the SimO model (dashed line) are quite close, while the detailed distribution of relevant observables is much different for each model, as we see next. 


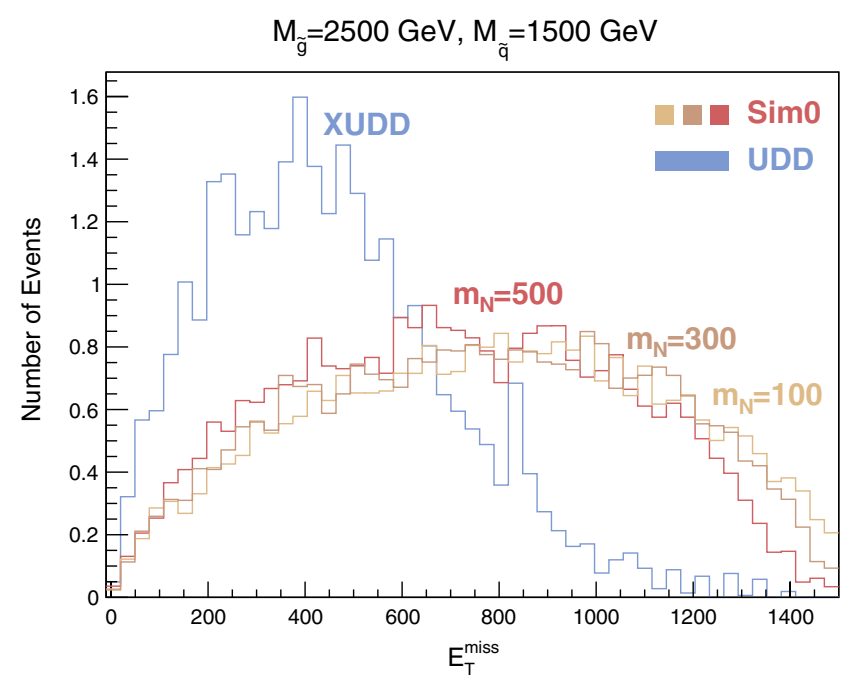

(a) $E_{T}^{\text {miss }}$

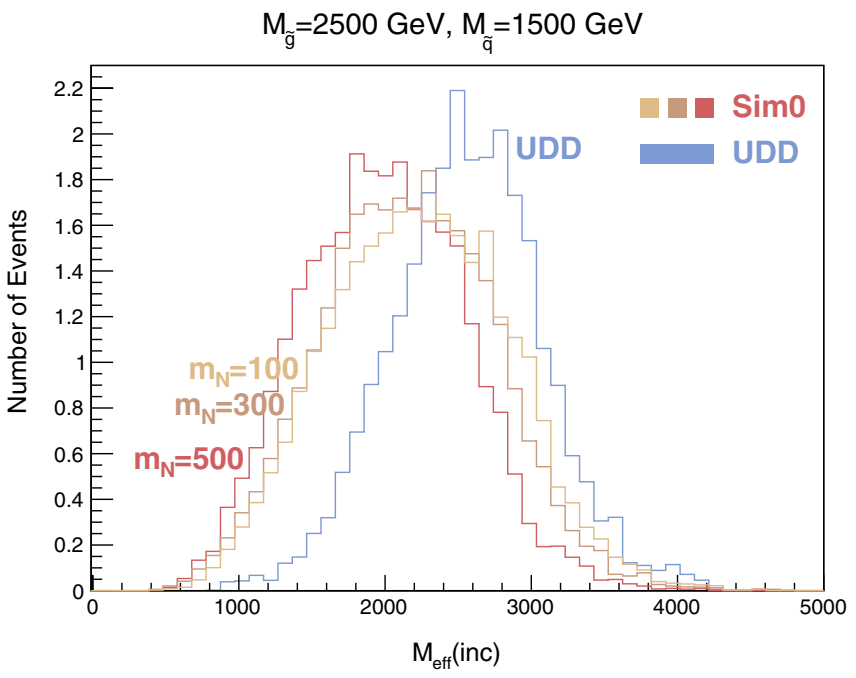

(c) $m_{\text {eff }}$ (incl.)

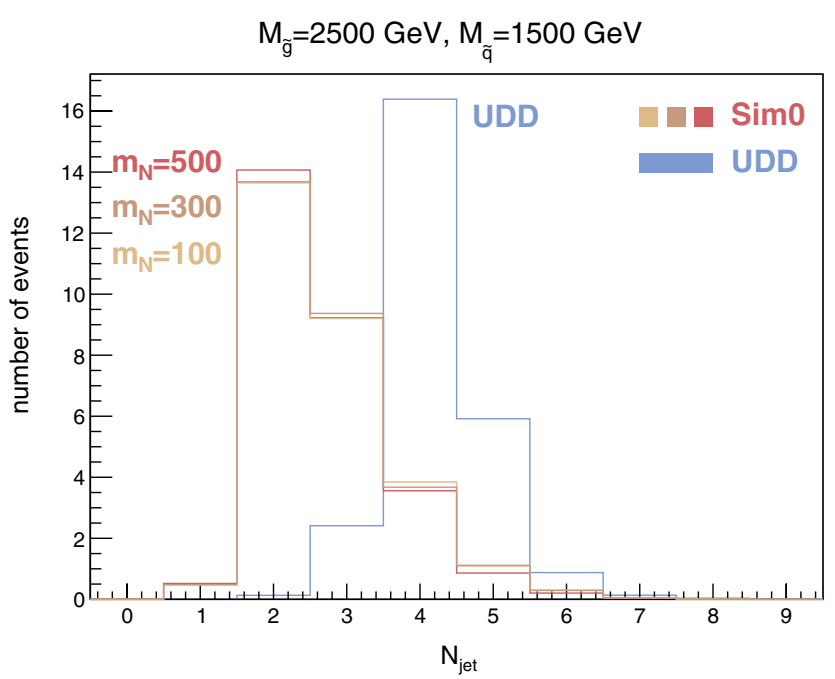

(b) $N_{\text {jet }}$

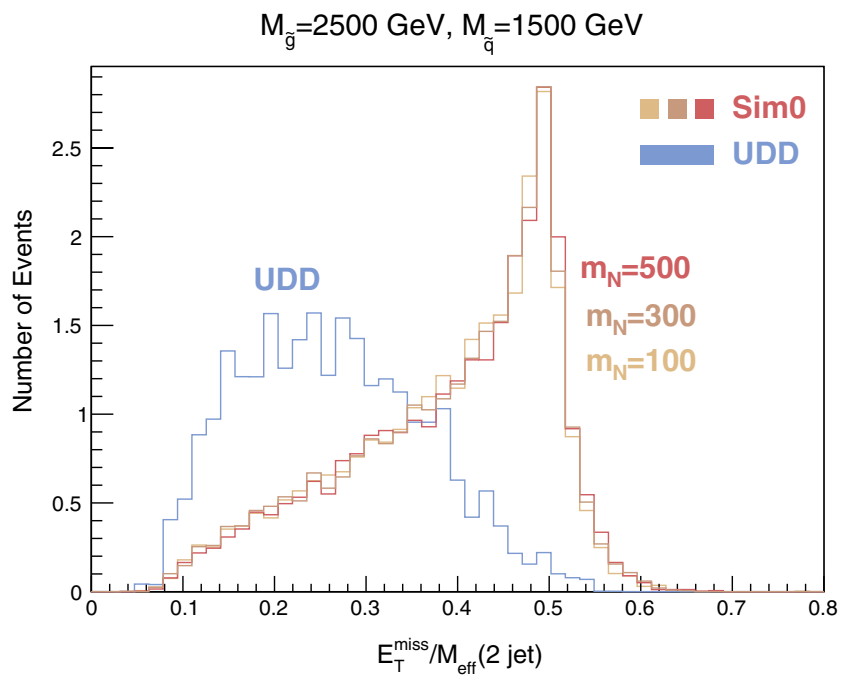

(d) $E_{T}^{\text {miss }} / m_{\text {eff }}(2 j)$

FIG. 18 (color online). Various distributions for the $u^{c} d^{c} d^{c}$ model with a squark LOSP (blue histograms) compared to the simplified model Sim0 (red histograms): missing transverse energy $E_{T}^{\text {miss }}$, number of jets $N_{\text {jets }}$ with $p_{T}>60 \mathrm{GeV}$ (for the hardest jet, $p_{T}>130 \mathrm{GeV}$ ), inclusive effective mass $m_{\text {eff }}$ (incl.) and $E_{T}^{\text {miss }} / m_{\text {eff }}(2 j)$. The mass parameters here are $m_{\tilde{g}}=2500 \mathrm{GeV}$, $m_{\tilde{q}}=1500 \mathrm{GeV}$. For the simplified model Sim0, we show three different neutralino masses, $m_{\chi_{1}^{0}}=100,300$ and $500 \mathrm{GeV}$.

In Fig. 18, we show the $E_{T}^{\text {miss }}, N_{\text {jet }}, m_{\text {eff }}$ (incl.) and $E_{T}^{\text {miss }} / m_{\text {eff }}(2 j)$ distributions for both models at $m_{\tilde{g}}=$ $2500 \mathrm{GeV}$ and $m_{\tilde{q}}=1500 \mathrm{GeV}$. The chosen mass parameter set is near the limit of the experimental sensitivity. Here, $m_{\text {eff }}$ (incl.) and $m_{\text {eff }}(2 j)$ are the effective mass defined inclusively, and exclusively with the two hardest jets, respectively. For $\operatorname{Sim} 0$, we show three different neutralino masses: $m_{\chi_{1}^{0}}=100,300$ and $500 \mathrm{GeV}$. For each histogram, we apply cuts in the 0-lepton analysis, similarly to the case of Fig. 12: After signal object identification/ isolation, we apply the lepton veto, and the two hardest jet $p_{T}$ cuts: $p_{T}\left(j_{1}\right)>130 \mathrm{GeV}, p_{T}\left(j_{2}\right)>60 \mathrm{GeV}$ for the MET distribution, and additionally the MET cut $E_{T}^{\text {miss }}>$ $160 \mathrm{GeV}$ for the other distributions.
One sees that the actual kinematic distributions are much different between the simplified and $u^{c} d^{c} d^{c}$ models. Nonetheless, the reason why the $u^{c} d^{c} d^{c}$ model and $\operatorname{Sim} 0$ have similar constraints is due to the saturation of the cut acceptance. Near the 95\% C.L. experimental sensitivity, the cuts in Table II are not very effective in distinguishing one model from another since the $p_{T}$ of relevant objects and the MET are already very high. The channels with harder cuts (for example, BT and CT) do not dominate the constraints and hence do not distinguish between models. For example, the acceptance of the AL channel cut is saturated above $m_{\tilde{q}}=1000 \mathrm{GeV}$ for a fixed gluino mass $m_{\tilde{g}}=2500 \mathrm{GeV}$, to $\sim 0.5$ for $u^{c} d^{c} d^{c}$ and $\sim 0.75$ for Sim0. Then, the constraints are simply determined by the production cross section, which is identical for both models. 


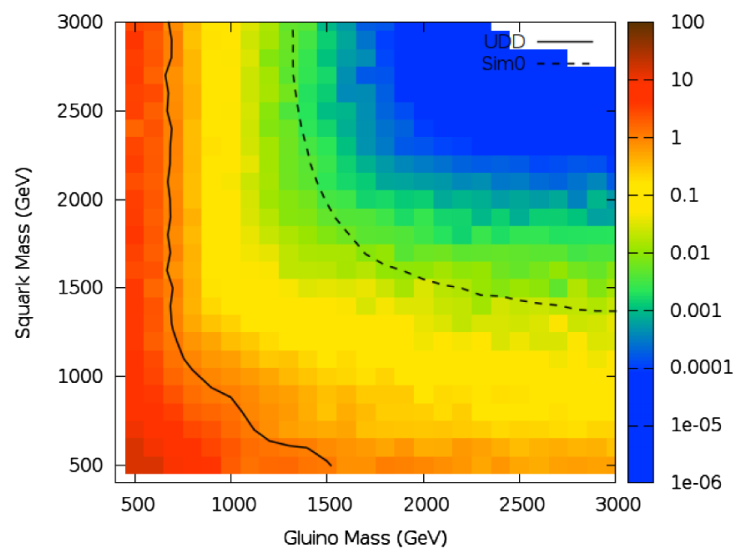

(a) 0 lepton analysis for $m_{\chi_{1}^{0}}=100 \mathrm{GeV}$

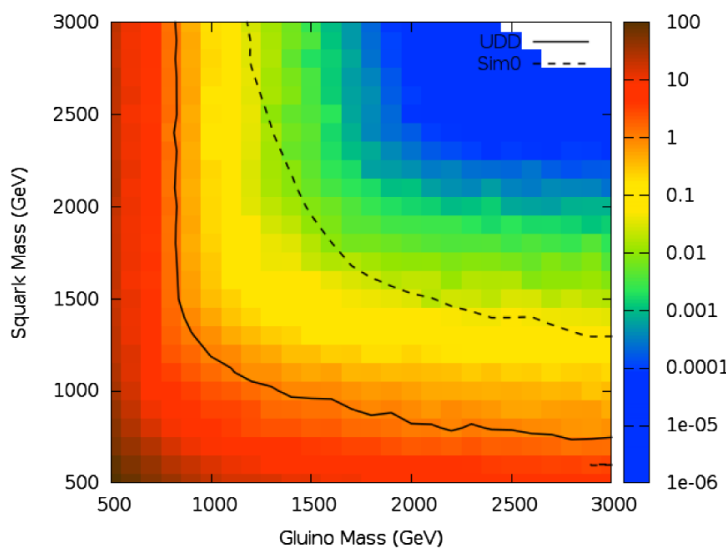

(b) 0 lepton analysis for $m_{\chi_{1}^{0}}=300 \mathrm{GeV}$

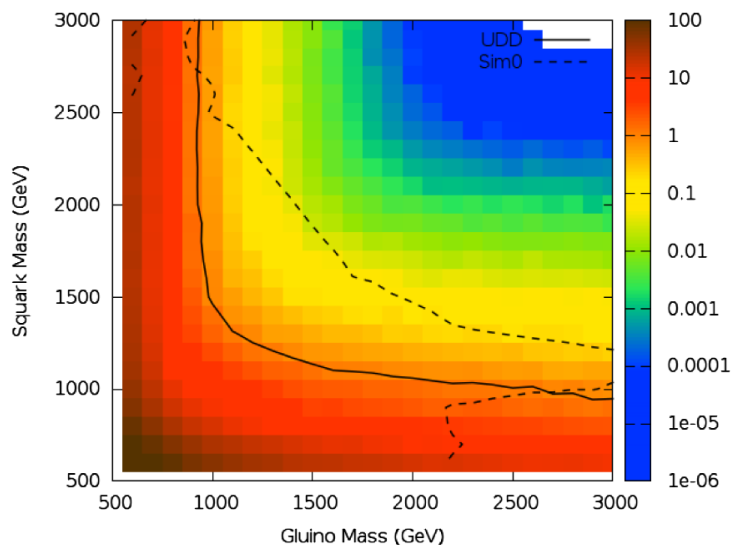

(c) 0 lepton analysis for $m_{\chi_{1}^{0}}=500 \mathrm{GeV}$

FIG. 19 (color online). Constraint from ATLAS 0-lepton + 2-6-jet + MET analysis on the $u^{c} d^{c} d^{c}$ model with a neutralino LOSP (solid curve), compared to the simplified model Sim0 (dashed curve) with the neutralino mass (a) $100 \mathrm{GeV}$, (b) $300 \mathrm{GeV}$, and (c) $500 \mathrm{GeV}$.

It is clear, however, that additional shape information from the kinematic distributions in Fig. 18 is available for discrimination between the simplified model and ADM, so the analysis could be better targeted to ADM models.

Neutralino LOSP.-Next we consider the $u^{c} d^{c} d^{c}$ model with a neutralino LOSP via the diagrams of Fig. 10 with the lepton or neutrino replaced with a jet. The results are shown in Fig. 19. In this case, as for the qedc model with a neutralino LOSP, we do not have to assume a splitting between squarks since squarks decay promptly into the neutralino. The $\left(m_{\tilde{g}}, m_{\tilde{q}}\right)$ scan results of the ATLAS 0-lepton analysis are shown in Fig. 19 for three different neutralino mass choices: $m_{\chi_{1}^{0}}=100,300$ and $500 \mathrm{GeV}$. Again, we compare the result of the $u^{c} d^{c} d^{c}$ model with the 0-lepton analysis of the simplified model Sim0 with the same neutralino mass parameters. The contours of $\operatorname{maximum}_{i}\left(S_{i} / S_{\exp , i}^{95}\right)=1$ for $u^{c} d^{c} d^{c}$ and Sim0 are drawn as solid and dashed curves, respectively.

The constraints for the neutralino LOSP $u^{c} d^{c} d^{c}$ model are generically weaker than the simplified model SimO for small $m_{\chi_{1}^{0}}(100$ and $300 \mathrm{GeV})$ but reveal a more complicated behavior in the $m_{\chi_{1}^{0}}=500 \mathrm{GeV}$ case. Several factors contribute to these results. One obvious factor that tends to give weaker constraints on the ADM model in the 0-lepton analysis is that the missing energy of the neutralino is reduced as it decays to three additional jets, as shown in Fig. 20. This feature is transparently comparable with the simplified model SimO since both models share the same event topology before the neutralino decay. On the other hand, as the neutralino mass is set heavier, the energy of the jets from gluino/ squark decay into the neutralino becomes smaller as the mass difference shrinks. Therefore, the experimental sensitivity to the simplified model $\operatorname{SimO}$ (and ordinary $R$-parity conserving MSSM scenarios, generically) is reduced for a heavier neutralino mass, while the ADM models are subject to more severe constraints since a massive neutralino is able to "store" and transfer energy to the ADM particle. Therefore, for large neutralino mass, the ADM model can actually become substantially more constrained than the simplified model. 


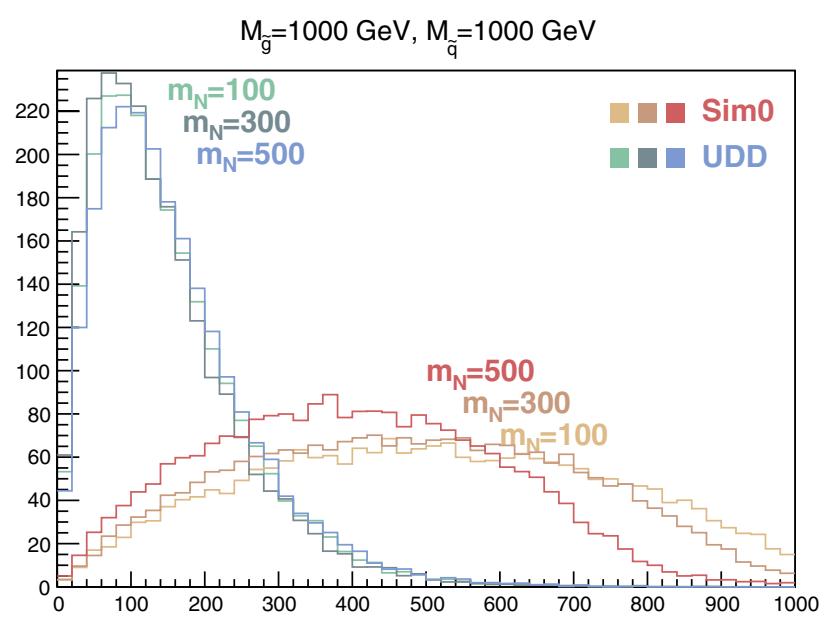

(a) $E_{T}^{\text {miss }}$

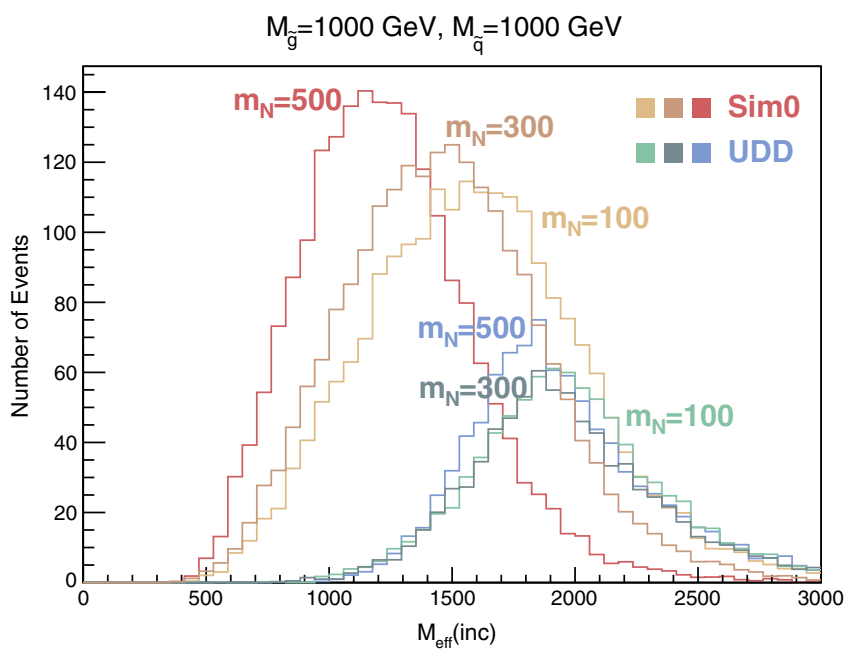

(c) $m_{\text {eff }}$ (incl.)

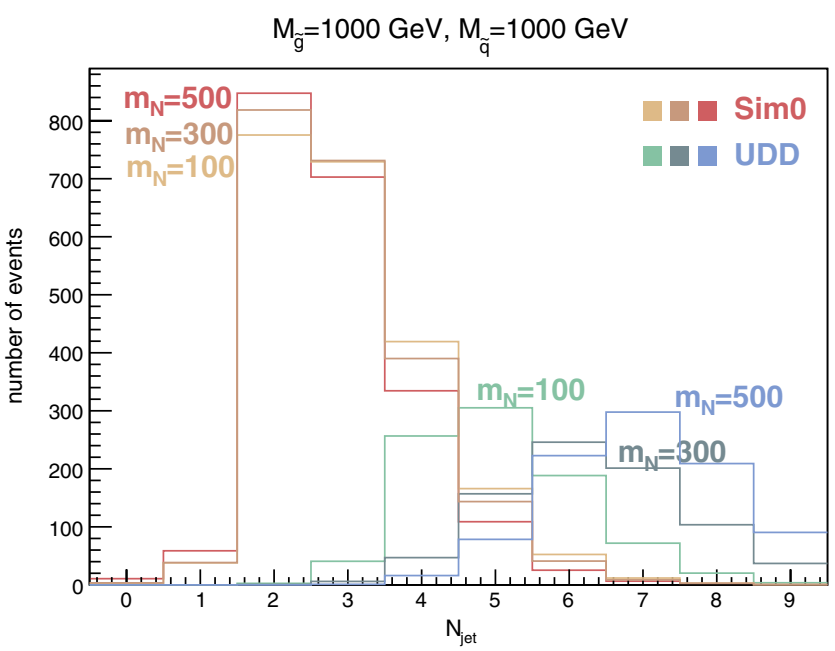

(b) $N_{\text {jet }}$

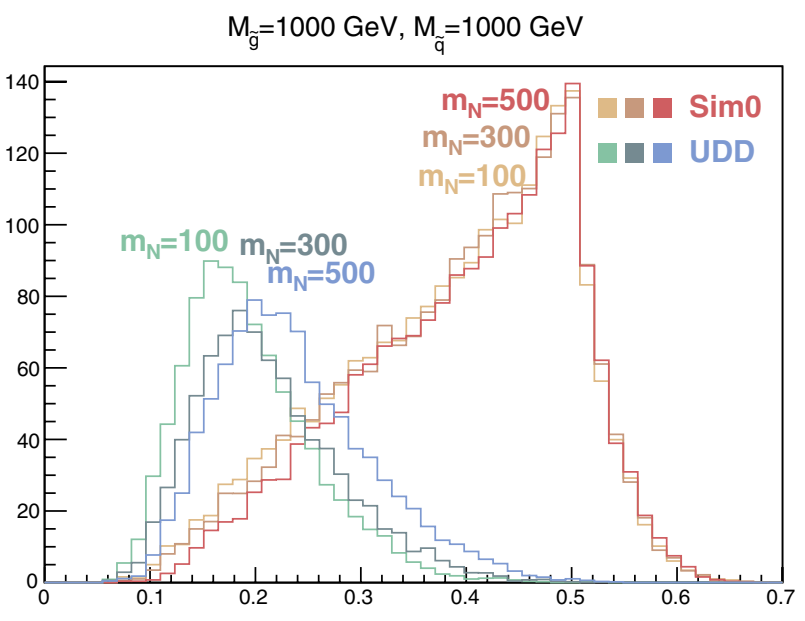

(d) $E_{T}^{\text {miss }} / m_{\text {eff }}(2 j)$

FIG. 20 (color online). Various distributions for the $u^{c} d^{c} d^{c}$ model with neutralino LOSP (blue histograms) compared to the simplified model Sim0 (red histograms): (a) missing transverse energy $E_{T}^{\text {miss }}$, (b) number of jets $N_{\text {jets }}$ with $p_{T}>60 \mathrm{GeV}$ (for the hardest jet, $p_{T}>130 \mathrm{GeV}$ ), (c) inclusive effective mass $m_{\text {eff }}$ (incl.) and (d) $E_{T}^{\text {miss }} / m_{\text {eff }}(2 j)$. The mass parameters here are $m_{\tilde{g}}=m_{\tilde{q}}=1000 \mathrm{GeV}$. For each model, we show three different neutralino masses, $m_{\chi_{1}^{0}}=100,300$ and $500 \mathrm{GeV}$.

In Fig. 20, we compare the $E_{T}^{\text {miss }}, N_{\text {jet }}, m_{\text {eff }}$ (incl.) and $E_{T}^{\text {miss }} / m_{\text {eff }}(2 j)$ distributions of the neutralino LOSP $u^{c} d^{c} d^{c}$ model and the simplified model $\operatorname{Sim} 0$ for $m_{\tilde{g}}=m_{\tilde{q}}=$ $1000 \mathrm{GeV}$ and $m_{\chi_{1}^{0}}=100,300$ and $500 \mathrm{GeV}$. Here, we use the same cuts as in Fig. 18. Note that $E_{T}^{\text {miss }}$ is distinctively smaller and $m_{\text {eff }}$ is significantly higher for the $u^{c} d^{c} d^{c}$ ADM model than for the simplified model, indicating that the simplified model more easily passes the $m_{\text {eff }}$ requirement. The net effect is that the constraints on the ADM model are weaker than for the simplified model, though the ADM model becomes more constrained relative to the simplified model as $m_{\chi_{1}^{0}}$ increases. There are a couple of reasons why the ADM model constraints become stronger at larger neutralino mass. First, the number of hard jets in the ADM model increases, improving the sensitivity to the model for the channels which require a high multiplicity of jets. Second, the acceptance on the $E_{T}^{\text {miss }} / m_{\text {eff }}(2 j)$ cut improves markedly as the neutralino mass increases: $m_{\mathrm{eff}}(2 j)$ decreases as the energy stored in the neutralino increases.

Lastly, as we did for the $q \ell d^{c}$ operator, we comment on detecting the states of the UV completion of the ADM operator-flavor-violating signatures can also result from prompt decays of the new states $U$ and $D$. When these states decay to the DM, $U, D \rightarrow X+q$, the signatures look similar to squark or stop signatures of a jet or top quark plus missing energy. On the other hand, these states may have flavor-violating decays to pairs of quarks, which may include only the light quarks, but also may result in flavor-violating decays $U \rightarrow t j$ or $D \rightarrow b j$. A study of 
these signatures could give rise to additional constraints on ADM sectors.

\section{CONCLUSION AND OUTLOOK}

We have carried out the first detailed study of flavor constraints and collider signatures of asymmetric dark matter. We found that while flavor constraints from meson oscillations and lepton flavor conservation place significant requirements on the scale $M$ of the ADM operators, this scale $M$ is not so high that a variety of collider prompt decays of the LOSP into the $X$ sector, including exotic flavor combinations, could not arise. We applied two standard $8 \mathrm{TeV}$ LHC searches for SUSY to LOSP decays to ADM plus additional jets and leptons. These analyses involved 2-6 jets plus missing energy, or 1-2 leptons plus 3-6 jets and missing energy. We found that the constraints from these analyses, whether the LOSP is a squark, slepton, or neutralino, are somewhat weakened, depending on the spectrum, in comparison to the standard searches. However, the detailed kinematic distributions show a significant difference between the conventional SUSY models and the ADM models. This suggests that other SUSY searches at the LHC might be sensitive to the ADMextended MSSM, in particular searches which involve an extremely high multiplicity of jets $[38,39]$. It also suggests that dedicated searches tuned to ADM could significantly extend the reach at the LHC.

One of the interesting conclusions of this work is that the source of large flavor violation may not be much beyond our current reach. The suppression scale of the ADM operator could be as low as $10 \mathrm{TeV}$, and the leptoquark-type states being integrated out could be as low as $1 \mathrm{TeV}$. These states, when they decay to the ADM sector or to the visible sector, could give rise to exotic flavor-violating signatures. Performing ADM model analyses for other SUSY searches, e.g. high jet multiplicity searches, third-generation focused searches, and exotica searches (e.g. leptoquark searches) will provide a better understanding of the current status of ADM models. We aim to carry out this study in the future. It will also be interesting to design searches for ADM to learn how much the LHC reach can be extended. The wellmotivated, simple extension of an ADM sector shows interesting interplay between flavor physics and collider physics and opens new unexplored directions for LHC phenomenology.

\section{ACKNOWLEDGMENTS}

We thank Yuval Grossman, Michele Papucci and LianTao Wang for discussions and Anson Hook for pointing out a problem in one of the Monte Carlo tools that we employ. We thank an anonymous referee for pointing out possible flavor violation from squark mass splitting and for checking a typo in the formulas. We also thank the Theory Group at Lawrence Berkeley National Laboratory for hospitality while part of this work was being completed. This work is supported by NSF CAREER Grant No. PHY 1049896, by the DOE under Contract No. de-sc0007859, and also in part by the CERN-Korean Fellowship through the National Research Foundation of Korea.

\section{APPENDIX A: ONE-LOOP BOX DIAGRAM CORRECTION TO FLAVOR VIOLATION}

In this section, we present the full one-loop corrections through box diagrams for flavor-violating processes in (i) meson oscillations, (ii) $\mu-e$ conversion, (iii) $B_{s} \rightarrow$ $\ell^{+} \ell^{-}, b \rightarrow s \ell^{+} \ell^{-}$and (iv) $\mu \rightarrow 3 e$.

First we begin with the general loop functions that will be useful for calculating the diagrams for all relevant processes. For the box diagrams, it is convenient to split an internal fermion propagator into a chirality preserving part $\left(\propto \gamma^{\mu}\right)$ and a chirality flipping part in the fermion line. From the diagrams (a) and (b) shown in Fig. 21, we have effective operators

$$
\begin{aligned}
B_{o}= & (\operatorname{Sym})\left(\bar{\Psi}_{2 P} \gamma^{\mu} \Psi_{1 P}\right)\left(\bar{\Psi}_{3 P^{\prime}} \gamma_{\mu} \Psi_{4 P^{\prime}}\right) \\
& \times \frac{\lambda_{1} \lambda_{2} \lambda_{3} \lambda_{4}}{64 \pi^{2}} H\left(m_{F_{1}}, m_{F_{2}}, m_{\phi_{1}}, m_{\phi_{2}}\right) \\
B_{m}= & (\operatorname{Sym})\left(\bar{\Psi}_{2 \bar{P}} \Psi_{1 P}\right)\left(\bar{\Psi}_{3 P^{\prime}} \Psi_{4 \bar{P}^{\prime}}\right) \\
& \times \frac{\lambda_{1} \lambda_{2} \lambda_{3} \lambda_{4}}{16 \pi^{2}} m_{F_{1}} m_{F_{2}} K\left(m_{F_{1}}, m_{F_{2}}, m_{\phi_{1}}, m_{\phi_{2}}\right),
\end{aligned}
$$

where $m_{A}$ is the mass of the particle $\mathrm{A}$ in the loop, $\lambda_{i}$ 's are four couplings involved in the diagram and (Sym) is an appropriate symmetry factor if there are identical particles in final states. We denote the chirality of each particle by $P$, $P^{\prime}=L, R$ and $\bar{P}, \bar{P}^{\prime}$ for the opposite chirality, as shown in Fig. 21. Note that the contribution $B_{m}$ can be reinterpreted as a vector-vector current interaction due to Fierz identities:

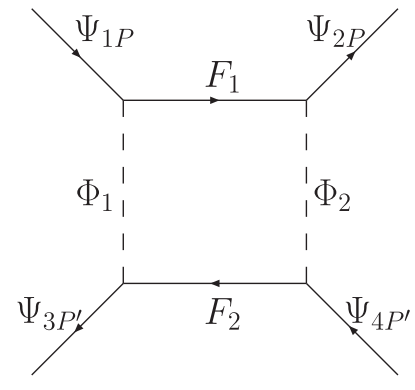

(a) $B_{O}$

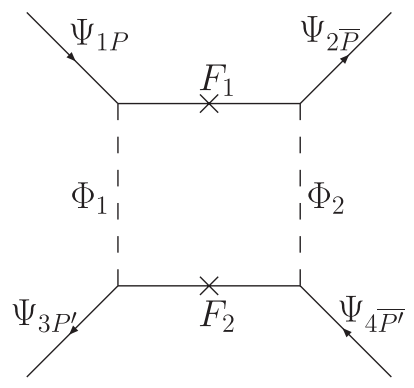

(b) $B_{M}$
FIG. 21. Box diagrams with a fermion line (a) without and (b) with a mass insertion. $P$ and $P^{\prime}$ denote chirality $L$ or $R$, and $\bar{P}$ and $\bar{P}^{\prime}$ are opposite chirality to $P$ and $P^{\prime}$, respectively. Here, we use four-component notation to easily match with Feynman amplitude expressions. 


$$
\left(\bar{\Psi}_{1 R} \Psi_{2 L}\right)\left(\bar{\Psi}_{3 L} \Psi_{4 R}\right)=-\frac{1}{2}\left(\bar{\Psi}_{1 R} \gamma^{\mu} \Psi_{4 R}\right)\left(\Psi_{3 L} \gamma_{\mu} \Psi_{2 L}\right)
$$

The loop functions in Eq. (A1) are defined by

$$
\begin{aligned}
H\left(m_{F_{1}}, m_{F_{2}}, m_{\phi_{1}}, m_{\phi_{2}}\right) \equiv & \frac{m_{F_{1}}^{4} \log \left(m_{F_{1}}^{2}\right)}{\left(m_{F_{1}}^{2}-m_{F_{2}}^{2}\right)\left(m_{F_{1}}^{2}-m_{\phi_{1}}^{2}\right)\left(m_{F_{1}}^{2}-m_{\phi_{2}}^{2}\right)}-\frac{m_{F_{2}}^{4} \log \left(m_{F_{2}}^{2}\right)}{\left(m_{F_{1}}^{2}-m_{F_{2}}^{2}\right)\left(m_{F_{2}}^{2}-m_{\phi_{1}}^{2}\right)\left(m_{F_{2}}^{2}-m_{\phi_{2}}^{2}\right)} \\
& +\frac{m_{\phi_{1}}^{4} \log m_{\phi_{1}}^{2}}{\left(m_{F_{1}}^{2}-m_{\phi_{2}}^{2}\right)\left(m_{F_{2}}^{2}-m_{\phi_{1}}^{2}\right)\left(m_{\phi_{1}}^{2}-m_{\phi_{2}}^{2}\right)}-\frac{m_{\phi_{2}}^{4} \log \left(m_{\phi_{2}}^{2}\right)}{\left(m_{F_{1}}^{2}-m_{\phi_{2}}^{2}\right)\left(m_{F_{2}}^{2}-m_{\phi_{2}}^{2}\right)\left(m_{\phi_{1}}^{2}-m_{\phi_{2}}^{2}\right)}, \\
K\left(m_{F_{1}}, m_{F_{2}}, m_{\phi_{1}}, m_{\phi_{2}}\right) \equiv & -\frac{m_{F_{1}}^{2} \log \left(m_{F_{1}}^{2}\right)}{\left(m_{F_{1}}^{2}-m_{F_{2}}^{2}\right)\left(m_{F_{1}}^{2}-m_{\phi_{1}}^{2}\right)\left(m_{F_{1}}^{2}-m_{\phi_{2}}^{2}\right)}+\frac{m_{F_{2}}^{2} \log \left(m_{F_{2}}^{2}\right)}{\left(m_{F_{1}}^{2}-m_{F_{2}}^{2}\right)\left(m_{F_{2}}^{2}-m_{\phi_{1}}^{2}\right)\left(m_{F_{2}}^{2}-m_{\phi_{2}}^{2}\right)} \\
& -\frac{m_{\phi_{1}}^{2} \log \left(m_{\phi_{1}}^{2}\right)}{\left(m_{F_{1}}^{2}-m_{\phi_{1}}^{2}\right)\left(m_{F_{2}}^{2}-m_{\phi_{1}}^{2}\right)\left(m_{\phi_{1}}^{2}-m_{\phi_{2}}^{2}\right)}+\frac{\left.m_{\phi_{2}}^{2}\right)}{\left(m_{F_{1}}^{2}-m_{\phi_{2}}^{2}\right)\left(m_{F_{2}}^{2}-m_{\phi_{2}}^{2}\right)\left(m_{\phi_{1}}^{2}-m_{\phi_{2}}^{2}\right)}
\end{aligned}
$$

For the loop contributions under consideration, we have $m_{F_{1}}=m_{F_{2}}$ or $m_{\phi_{1}}=m_{\phi_{2}}$ in most cases. If $m_{F_{1}}$ and $m_{F_{2}}$ are the same, $H$ and $K$ are given by

$$
\begin{aligned}
H\left(m_{F} ; m_{\phi_{1}}, m_{\phi_{2}}\right)= & \frac{m_{F}^{2}}{\left(m_{F}^{2}-m_{\phi_{1}}^{2}\right)\left(m_{F}^{2}-m_{\phi_{2}}^{2}\right)}-\frac{m_{F}^{2} \log \left(m_{F}^{2}\right)}{\left(m_{F}^{2}-m_{\phi_{1}}^{2}\right)\left(m_{F}^{2}-m_{\phi_{2}}^{2}\right)}\left[\frac{m_{\phi_{1}}^{2}}{m_{F}^{2}-m_{\phi_{1}}^{2}}+\frac{m_{\phi_{2}}^{2}}{m_{F}^{2}-m_{\phi_{2}}^{2}}\right] \\
& +\frac{m_{\phi_{1}}^{4} \log \left(m_{\phi_{1}}^{2}\right)}{\left(m_{\phi_{1}}^{2}-m_{\phi_{2}}^{2}\right)\left(m_{F}^{2}-m_{\phi_{1}}^{2}\right)^{2}}-\frac{m_{\phi_{2}}^{4} \log \left(m_{\phi_{2}}^{2}\right)}{\left(m_{\phi_{1}}^{2}-m_{\phi_{2}}^{2}\right)\left(m_{F}^{2}-m_{\phi_{2}}^{2}\right)^{2}}, \\
K\left(m_{F} ; m_{\varphi_{1}}, m_{\varphi_{2}}\right)= & -\frac{1}{\left(m_{F}^{2}-m_{\varphi_{1}}^{2}\right)\left(m_{F}^{2}-m_{\varphi_{2}}^{2}\right)}+\frac{\left(m_{F}^{4}-m_{\varphi_{1}}^{2} m_{\varphi_{2}}^{2}\right) \log \left(m_{F}^{2}\right)}{\left(m_{F}^{2}-m_{\varphi_{1}}^{2}\right)^{2}\left(m_{F}^{2}-m_{\varphi_{2}}^{2}\right)^{2}}-\frac{m_{\varphi_{1}}^{2} \log \left(m_{\varphi_{1}}^{2}\right)}{\left(m_{\varphi_{1}}^{2}-m_{\varphi_{2}}^{2}\right)\left(m_{F}^{2}-m_{\varphi_{1}}^{2}\right)^{2}} \\
& +\frac{m_{\varphi_{2}}^{2} \log \left(m_{\varphi_{2}}^{2}\right)}{\left(m_{\varphi_{1}}^{2}-m_{\varphi_{2}}^{2}\right)\left(m_{F}^{2}-m_{\varphi_{2}}^{2}\right)^{2}}
\end{aligned}
$$

and similarly for $m_{\phi_{1}}=m_{\phi_{2}}$. For $m_{F_{1}}=m_{F_{2}}$ and $m_{\phi_{1}}=m_{\phi_{2}}$, the loop functions are reduced to

$$
\begin{gathered}
H\left(m_{F} ; m_{\phi}\right)=\frac{m_{F}^{2}+m_{\phi}^{2}}{\left(m_{F}^{2}-m_{\phi}^{2}\right)^{2}}-\frac{2 m_{F}^{2} m_{\phi}^{2}}{\left(m_{F}^{2}-m_{\phi}^{2}\right)^{3}} \log \left(\frac{m_{F}^{2}}{m_{\phi}^{2}}\right), \\
K\left(m_{F} ; m_{\phi}\right)=-\frac{2}{\left(m_{F}^{2}-m_{\phi}^{2}\right)^{2}}+\frac{m_{F}^{2}+m_{\phi}^{2}}{\left(m_{F}^{2}-m_{\phi}^{2}\right)^{3}} \log \left(\frac{m_{F}^{2}}{m_{\phi}^{2}}\right) .
\end{gathered}
$$

In the following subsections, we present the corresponding expressions in the UV complete models for various flavor constraints.

\section{Meson mixing constraints}

Experimental constraints from $K-, D$-, $B$-meson mixing put stringent constraints on the UV models for the $X q \ell d^{c}$ and $X u^{c} d^{c} d^{c}$ operators. The effective operators generated from the models are summarized by the following effective Lagrangian: 
TABLE V. Flavor constraints from meson oscillations. The numbers are in TeV. The operator which is constrained is shown, along with the constraint on $\Lambda$ [25]. For the model $\Phi$ where $\Phi$ denotes a pair $\left(\Phi, \Phi^{c}\right)$ in the UV completion, $m_{\Phi} / \sqrt{\lambda^{4}}$ is constrained as shown in the table. Here, $R_{\Phi}=\log \left(m_{\Phi}^{2} / m_{\text {soft }}^{2}\right)-1$. For the model $L$ in $X q \ell d^{c}$, we show the tree-level contribution (boxed) for the $L R$ mixing operator for $K-\bar{K}, B_{d, s}-\bar{B}_{d, s}$. The constraints for the tree-level operator is implied for $m_{L} / \sqrt{\lambda^{2}}$. For the model $D$ in $X u^{c} d^{c} d^{c}$, the coupling combination $(*),(* *)$, and $(* * *)$ is presented in Eq. (A10).

\begin{tabular}{|c|c|c|c|c|c|}
\hline \multirow{2}{*}{$\begin{array}{l}\text { Operator } \\
\text { Limit }(\mathrm{TeV})\end{array}$} & \multicolumn{3}{|c|}{$\lambda^{4}\left(\lambda^{2}\right.$ for tree level) in $X q \ell d^{c}$} & \multicolumn{2}{|c|}{$X u^{c} d^{c} d^{c}$} \\
\hline & $D$ & $L$ & $Q$ & $U$ & $D$ \\
\hline$\overline{\left(\bar{s}_{R} \gamma^{\mu} d_{R}\right)^{2}}$ & $\left(\lambda_{X D}^{1} \lambda_{X D}^{2}\right)^{2}$ & $\left(\lambda_{L}^{i 1} \lambda_{L}^{i 2}\right)^{2}$ & $\left(\lambda_{Q}^{i 1} \lambda_{Q}^{i 2}\right)^{2}$ & $\left(\lambda_{U}^{13} \lambda_{U}^{23}\right)^{2}$ & $(*)$ \\
\hline 980 & 78 & 110 & 110 & 78 & 78 \\
\hline$\left(\bar{s}_{L} \gamma^{\mu} d_{L}\right)^{2}$ & $\left(\lambda_{D}^{i 1} \lambda_{D}^{i 2}\right)^{2}$ & $\left(\lambda_{L}^{1 i} \lambda_{L}^{2 i}\right)^{2}$ & $\left(\lambda_{X Q}^{1} \lambda_{X Q}^{2}\right)^{2}$ & & \\
\hline 980 & 78 & 78 & 78 & & \\
\hline$\left(\bar{s}_{L} d_{R}\right)\left(\bar{s}_{R} d_{L}\right)$ & $\lambda_{X D}^{1} \lambda_{X D}^{2} \lambda_{D}^{1 i} \lambda_{D}^{2 i} R_{D}$ & $\lambda_{L}^{12} \lambda_{L}^{21}, \lambda_{L}^{1 i} \lambda_{L}^{2 i} \lambda_{L}^{j 1} \lambda_{L}^{j 2}$ & $\lambda_{X Q}^{1} \lambda_{X Q}^{2} \lambda_{Q}^{i 1} \lambda_{Q}^{i 2} R_{Q}$ & & \\
\hline 18000 & 1400 & 18000,990 & 1400 & & \\
\hline$\left(\bar{c}_{R} \gamma^{\mu} u_{R}\right)^{2}$ & & & & $\left(\lambda_{X U}^{1} \lambda_{X U}^{2}\right)^{2}$ & $\left(\lambda_{D}^{1 i} \lambda_{D}^{2 i}\right)^{2}$ \\
\hline 1200 & & & & 95 & 95 \\
\hline$\left(\bar{c}_{L} \gamma^{\mu} u_{L}\right)^{2}$ & $\left(\lambda_{D}^{1 i} \lambda_{D}^{2 i}\right)^{2}$ & $\left(\lambda_{L}^{1 i} \lambda_{L}^{2 i}\right)^{2}$ & $\left(\lambda_{X Q}^{1} \lambda_{X Q}^{2}\right)^{2}$ & & \\
\hline 1200 & 95 & 95 & 95 & & \\
\hline$\left(\bar{b}_{R} \gamma^{\mu} d_{R}\right)^{2}$ & $\left(\lambda_{X D}^{1} \lambda_{X D}^{3}\right)^{2}$ & $\left(\lambda_{L}^{i 1} \lambda_{L}^{i 3}\right)^{2}$ & $\left(\lambda_{Q}^{i 1} \lambda_{Q}^{i 3}\right)^{2}$ & $\left(\lambda_{U}^{12} \lambda_{U}^{23}\right)^{2}$ & $(* *)$ \\
\hline 510 & 41 & 57 & 57 & 41 & 41 \\
\hline$\left(\bar{b}_{L} \gamma^{\mu} d_{L}\right)^{2}$ & $\left(\lambda_{D}^{i 1} \lambda_{D}^{i 3}\right)^{2}$ & $\left(\lambda_{L}^{1 i} \lambda_{L}^{3 i}\right)^{2}$ & $\left(\lambda_{X Q}^{1} \lambda_{X Q}^{3}\right)^{2}$ & & \\
\hline 510 & 41 & 41 & 41 & & \\
\hline$\left(\bar{b}_{L} d_{R}\right)\left(\bar{b}_{R} d_{L}\right)$ & $\lambda_{X D}^{1} \lambda_{X D}^{3} \lambda_{D}^{1 i} \lambda_{D}^{3 i} R_{D}$ & $\lambda_{L}^{13} \lambda_{L}^{31}, \lambda_{L}^{1 i} \lambda_{L}^{3 i} \lambda_{L}^{j 1} \lambda_{L}^{j 3}$ & $\lambda_{X Q}^{1} \lambda_{X Q}^{3} \lambda_{Q}^{i 1} \lambda_{Q}^{i 3} R_{Q}$ & & \\
\hline 1900 & 151 & 1900, 110 & 151 & & \\
\hline$\left(\bar{b}_{R} \gamma^{\mu} s_{R}\right)^{2}$ & $\left(\lambda_{X D}^{2} \lambda_{X D}^{3}\right)^{2}$ & $\left(\lambda_{L}^{i 2} \lambda_{L}^{i 3}\right)^{2}$ & $\left(\lambda_{Q}^{i 2} \lambda_{Q}^{i 3}\right)^{2}$ & $\left(\lambda_{U}^{12} \lambda_{U}^{13}\right)^{2}$ & $(* * *)$ \\
\hline 110 & 8.7 & 12 & 12 & 8.7 & 8.7 \\
\hline$\left(\bar{b}_{L} \gamma^{\mu} s_{L}\right)^{2}$ & $\left(\lambda_{D}^{i 2} \lambda_{D}^{i 3}\right)^{2}$ & $\left(\lambda_{L}^{2 i} \lambda_{L}^{3 i}\right)^{2}$ & $\left(\lambda_{X Q}^{2} \lambda_{X Q}^{3}\right)^{2}$ & & \\
\hline 110 & 8.7 & 8.7 & 8.7 & & \\
\hline$\left(\bar{b}_{L} s_{R}\right)\left(\bar{b}_{R} s_{L}\right)$ & $\lambda_{X D}^{2} \lambda_{X D}^{3} \lambda_{D}^{2 i} \lambda_{D}^{3 i} R_{D}$ & $\lambda_{L}^{23} \lambda_{L}^{32}, \lambda_{L}^{2 i} \lambda_{L}^{3 i} \lambda_{L}^{j 2} \lambda_{L}^{j 3}$ & $\lambda_{X Q}^{2} \lambda_{X Q}^{3} \lambda_{Q}^{i 2} \lambda_{Q}^{i 3} R_{Q}$ & & \\
\hline 370 & 29 & 370,21 & 29 & & \\
\hline
\end{tabular}

$$
\begin{aligned}
\mathcal{L}_{\text {eff }}= & \frac{1}{4} K_{R R}\left(\bar{d}_{R} \gamma^{\mu} s_{R}\right)\left(\bar{d}_{R} \gamma_{\mu} s_{R}\right)+\frac{1}{4} K_{L L}\left(\bar{d}_{L} \gamma^{\mu} s_{L}\right)\left(\bar{d}_{L} \gamma_{\mu} s_{L}\right)+K_{L R}\left(\bar{s}_{L} d_{R}\right)\left(\bar{s}_{R} d_{L}\right)+\frac{1}{4} D_{R R}\left(\bar{c}_{R} \gamma^{\mu} u_{R}\right)\left(\bar{c}_{R} \gamma_{\mu} u_{R}\right) \\
& +\frac{1}{4} D_{L L}\left(\bar{c}_{L} \gamma^{\mu} u_{L}\right)\left(\bar{c}_{L} \gamma_{\mu} u_{L}\right)+\frac{1}{4} B_{d R R}\left(\bar{d}_{R} \gamma^{\mu} b_{R}\right)\left(\bar{d}_{R} \gamma_{\mu} b_{R}\right)+\frac{1}{4} B_{d L L}\left(\bar{d}_{L} \gamma^{\mu} b_{L}\right)\left(\bar{d}_{L} \gamma_{\mu} b_{L}\right)+B_{d L R}\left(\bar{b}_{L} d_{R}\right)\left(\bar{b}_{R} d_{L}\right) \\
& +\frac{1}{4} B_{s R R}\left(\bar{s}_{R} \gamma^{\mu} b_{R}\right)\left(\bar{s}_{R} \gamma_{\mu} b_{R}\right)+\frac{1}{4} B_{s L L}\left(\bar{s}_{L} \gamma^{\mu} b_{L}\right)\left(\bar{s}_{L} \gamma_{\mu} b_{L}\right)+B_{s L R}\left(\bar{b}_{L} s_{R}\right)\left(\bar{b}_{R} s_{L}\right),
\end{aligned}
$$

where $K_{P P^{\prime}}, D_{P P^{\prime}}, B_{d P P^{\prime}}$ and $B_{s P P^{\prime}}$ are the coefficients of the corresponding operators. For $B_{d P P^{\prime}}$ and $B_{s P P^{\prime}}$, the results can be easily read from $K_{P P^{\prime}}$ by changing the generation index to a $b$ quark, so we will omit them in the following.

Under the assumption that $m_{X} \sim m_{\tilde{x}} \ll m_{\text {soft }} \ll m_{D}, m_{L}, m_{Q}, m_{U}$, we summarize the tree-level and one-loop-level constraints on the mass and the coupling from meson mixing in Table V. In the table, $R_{\Phi}$ denotes $\log \left(m_{\Phi}^{2} / m_{\text {soft }}^{2}\right)-1$. The coupling combinations for $R R$ operators for $K$ - and $B$-meson mixing in the $D \mathrm{UV}$ completion for $X u^{c} d^{c} d^{c}$ are given by

$$
\begin{gathered}
(*)=\left(\lambda_{X D}^{1} \lambda_{X D}^{2}\right)^{2}+\left(\lambda_{D}^{i 1} \lambda_{D}^{i 2}\right)^{2}-2 \lambda_{X D}^{1} \lambda_{X D}^{2} \lambda_{D}^{i 1} \lambda_{D}^{i 2} R_{D}, \quad(* *)=\left(\lambda_{X D}^{1} \lambda_{X D}^{2}\right)^{3}+\left(\lambda_{D}^{i 1} \lambda_{D}^{i 3}\right)^{2}-2 \lambda_{X D}^{1} \lambda_{X D}^{3} \lambda_{D}^{i 1} \lambda_{D}^{i 3} R_{D}, \\
(* * *)=\left(\lambda_{X D}^{2} \lambda_{X D}^{3}\right)^{2}+\left(\lambda_{D}^{i 2} \lambda_{D}^{i 3}\right)^{2}-2 \lambda_{X D}^{2} \lambda_{X D}^{3} \lambda_{D}^{i 2} \lambda_{D}^{i 3} R_{D} .
\end{gathered}
$$


TABLE VI. Flavor constraints from $\mu-e$ conversion for the $X q \ell d^{c}$ models. Each row represents the UV completion. The numbers below the couplings are the constraints on $\Lambda \mathrm{in} \mathrm{TeV}$, which are $m_{\Phi} / \sqrt{\lambda^{2}}$ for the tree-level contribution and $m_{\Phi} / \sqrt{\lambda^{4}}$ for the one-loop contribution for the model $\Phi$.

\begin{tabular}{|c|c|c|}
\hline$D$ & Tree $\lambda^{2}$ & One loop $\lambda^{4}$ \\
\hline & $\begin{array}{l}\lambda_{D}^{11} \lambda_{D}^{12} \\
290 \mathrm{TeV}\end{array}$ & $\begin{array}{c}\frac{3}{2} \lambda_{D}^{i 1} \lambda_{D}^{i 2} \lambda_{D}^{1 j} \lambda_{D}^{1 j}-\frac{1}{2} \lambda_{D}^{i 1} \lambda_{D}^{i 2} \lambda_{X D}^{1} \lambda_{X D}^{2} R_{D} \\
23 \mathrm{TeV}\end{array}$ \\
\hline$L$ & & $\begin{array}{c}\lambda_{X L}^{1} \lambda_{X L}^{2}\left(\frac{1}{2} \lambda_{L}^{i 1} \lambda_{L}^{i 1}+\lambda_{L}^{1 i} \lambda_{L}^{i 1}\right) R_{L} \\
23 \mathrm{TeV}\end{array}$ \\
\hline$Q$ & $\begin{array}{c}\lambda_{Q}^{11} \lambda_{Q}^{21} \\
210 \mathrm{TeV}\end{array}$ & $\begin{array}{c}\frac{1}{2} \lambda_{Q}^{1 i} \lambda_{Q}^{2 i} \lambda_{Q}^{j 1} \lambda_{Q}^{j 1}-\lambda_{Q}^{1 i} \lambda_{Q}^{2 i} \lambda_{X Q}^{1} \lambda_{X Q}^{2} R_{Q} \\
23 \mathrm{TeV}\end{array}$ \\
\hline
\end{tabular}

\section{a. $X q \ell d^{c}$}

Since we have three classes of UV completions for the operator $X q \ell d^{c}$, we specify the contribution from the model $M$ by putting a superscript $(M)$ in the following. First, we present the contributions from the UV completion of the $X q \ell d^{c}$ operator.

For the model $(D)$ defined by Eq. (2.2), we obtain the operators for kaon physics,

$$
\begin{aligned}
K_{R R}^{(D)} & =\frac{\left(\lambda_{X D}^{1} \lambda_{X D}^{2}\right)^{2}}{64 \pi^{2}}\left[2 H\left(m_{X}, m_{X}, m_{\tilde{D}}, m_{\tilde{D}}\right)+2 H\left(m_{D}, m_{D}, m_{\tilde{x}}, m_{\tilde{x}}\right)\right], \\
K_{L L}^{(D)} & =\frac{\lambda_{D}^{i 1} \lambda_{D}^{i 2} \lambda_{D}^{j 1} \lambda_{D}^{j 2}}{64 \pi^{2}}\left[2 H\left(m_{D}, m_{D}, m_{\tilde{\nu}^{i}}, m_{\tilde{\nu}^{j}}\right)+2 H\left(m_{\nu^{i}}, m_{\nu^{j}}, m_{\tilde{D}}, m_{\tilde{D}}\right)\right], \\
K_{L R}^{(D)} & =\frac{\lambda_{D}^{1 i} \lambda_{D}^{2 i} \lambda_{X D}^{1} \lambda_{X D}^{2}}{16 \pi^{2}} m_{D}^{2} K\left(m_{D}, m_{D}, m_{\tilde{\nu}^{i}}, m_{\tilde{x}}\right),
\end{aligned}
$$

and for $D$-meson physics,

$$
D_{R R}^{(D)}=0, \quad D_{L L}^{(D)}=\frac{\lambda_{D}^{1 i} \lambda_{D}^{2 i} \lambda_{D}^{1 j} \lambda_{D}^{2 j}}{64 \pi^{2}}\left[2 H\left(m_{D}, m_{D}, m_{\tilde{e}^{i}}, m_{\tilde{e}^{j}}\right)+2 H\left(m_{e^{i}}, m_{e^{j}}, m_{\tilde{D}}, m_{\tilde{D}}\right)\right] .
$$

For the model $(L)$ from Eq. (2.3), we have

$$
\begin{aligned}
K_{R R}^{(L)} & =\frac{\lambda_{L}^{i 1} \lambda_{L}^{i 2} \lambda_{L}^{j 1} \lambda_{L}^{j 2}}{64 \pi^{2}}\left[2 H\left(m_{d^{i}}, m_{d^{j}}, m_{\tilde{L}}, m_{\tilde{L}}\right)+2 H\left(m_{L}, m_{L}, m_{\tilde{d}^{i}}, m_{\tilde{d}^{j}}\right)+2 H\left(m_{u^{i}}, m_{u^{j}}, m_{\tilde{L}}, m_{\tilde{L}}\right)+2 H\left(m_{L}, m_{L}, m_{\tilde{u}^{i}}, m_{\tilde{u}^{j}}\right)\right], \\
K_{L L}^{(L)} & =\frac{\lambda_{L}^{1 i} \lambda_{L}^{2 i} \lambda_{L}^{1 j} \lambda_{L}^{2 j}}{64 \pi^{2}}\left[2 H\left(m_{L}, m_{L}, m_{\tilde{d}^{c}}, m_{\tilde{d}^{c j}}\right)+2 H\left(m_{d^{i}}, m_{d^{j}}, m_{\tilde{L}}, m_{\tilde{L}}\right)\right] \\
K_{L R}^{(L)} & =\frac{\lambda_{L}^{1 i} \lambda_{L}^{2 i} \lambda_{L}^{j 1} \lambda_{L}^{j 2}}{64 \pi^{2}}\left[H\left(m_{d^{i}}, m_{d^{j}}, m_{\tilde{L}}, m_{\tilde{L}}\right)+H\left(m_{L}, m_{L}, m_{\tilde{d}^{c i}}, m_{\tilde{d}^{c j}}\right)\right], D_{R R}^{(L)}=0 \\
D_{L L}^{(L)} & =\frac{\lambda_{L}^{1 i} \lambda_{L}^{2 i} \lambda_{L}^{1 j} \lambda_{L}^{2 j}}{64 \pi^{2}}\left[2 H\left(m_{L}, m_{L}, m_{\tilde{d}^{c i}}, m_{\tilde{d}^{c j}}\right)+2 H\left(m_{d^{i}}, m_{d^{j}}, m_{\tilde{L}}, m_{\tilde{L}}\right)\right] .
\end{aligned}
$$

Now, we show the result for the model $(Q)$ :

$K_{R R}^{(Q)}=\frac{\lambda_{Q}^{i 1} \lambda_{Q}^{i 2} \lambda_{Q}^{j 1} \lambda_{Q}^{j 2}}{64 \pi^{2}}\left[2 H\left(m_{e^{i}}, m_{e^{j}}, m_{\tilde{Q}}, m_{\tilde{Q}}\right)+2 H\left(m_{Q}, m_{Q}, m_{\tilde{e}^{i}}, m_{\tilde{e}^{j}}\right)+2 H\left(m_{\nu^{i}}, m_{\nu^{j}}, m_{\tilde{Q}}, m_{\tilde{Q}}\right)+2 H\left(m_{Q}, m_{Q}, m_{\tilde{\nu}^{i}}, m_{\tilde{\nu}^{j}}\right)\right]$, 


$$
\begin{aligned}
K_{L L}^{(Q)}= & \frac{\left(\lambda_{X Q}^{1} \lambda_{X Q}^{2}\right)^{2}}{64 \pi^{2}}\left[2 H\left(m_{X}, m_{X}, m_{\tilde{Q}}, m_{\tilde{Q}}\right)\right. \\
& \left.+2 H\left(m_{Q}, m_{Q}, m_{\tilde{x}}, m_{\tilde{x}}\right)\right], \\
K_{L R}^{(Q)}= & \frac{\lambda_{X Q}^{1} \lambda_{X Q}^{2} \lambda_{Q}^{i 1} \lambda_{Q}^{i 2}}{16 \pi^{2}} m_{Q}^{2} K\left(m_{Q}, m_{Q}, m_{\tilde{x}}, m_{\tilde{\nu}^{i}}\right), \\
D_{R R}^{(Q)}= & 0, D_{L L}^{(Q)} \\
= & \frac{\left(\lambda_{X Q}^{1} \lambda_{X Q}^{2}\right)^{2}}{64 \pi^{2}}\left[2 H\left(m_{X}, m_{X}, m_{\tilde{Q}}, m_{\tilde{Q}}\right)\right. \\
& \left.+2 H\left(m_{Q}, m_{Q}, m_{\tilde{x}}, m_{\tilde{x}}\right)\right] .
\end{aligned}
$$

\section{b. $X u^{c} d^{c} d^{c}$}

As in the $X q \ell d^{c}$ case, we specify each model by the superscripts $(U)$ and $(D)$. For the model $(U)$,

$$
\begin{aligned}
K_{R R}^{(U)}= & \frac{\left(\lambda_{U}^{13} \lambda_{U}^{23}\right)^{2}}{64 \pi^{2}}\left[2 H\left(m_{U}, m_{U}, m_{\tilde{d}^{c 3}}, m_{\tilde{d}^{c 3}}\right)\right. \\
& \left.+2 H\left(m_{b}, m_{b}, m_{\tilde{U}}, m_{\tilde{U}}\right)\right], \\
D_{R R}^{(U)}= & \frac{\left(\lambda_{X U}^{1} \lambda_{X U}^{2}\right)^{2}}{64 \pi^{2}}\left[2 H\left(m_{X}, m_{X}, m_{\tilde{U}}, m_{\tilde{U}}\right)\right. \\
& \left.+2 H\left(m_{U}, m_{U}, m_{\tilde{x}}, m_{\tilde{x}}\right)\right], \\
K_{L L}^{(U)}= & K_{L R}^{(U)}=D_{L L}^{(U)}=0,
\end{aligned}
$$

and for the model $(D)$,

$$
\begin{aligned}
K_{R R}^{(D)}= & \frac{\left(\lambda_{X D}^{1} \lambda_{X D}^{2}\right)^{2}}{64 \pi^{2}}\left[2 H\left(m_{X}, m_{X}, m_{\tilde{D}}, m_{\tilde{D}}\right)\right. \\
& \left.+2 H\left(m_{D}, m_{D}, m_{\tilde{x}}, m_{\tilde{x}}\right)\right] \\
& +\frac{\lambda_{D}^{i 1} \lambda_{D}^{i 2} \lambda_{D}^{j 1} \lambda_{D}^{j 2}}{64 \pi^{2}}\left[2 H\left(m_{u^{i}}, m_{u^{j}}, m_{\tilde{D}}, m_{\tilde{D}}\right)\right. \\
& \left.+2 H\left(m_{D}, m_{D}, m_{\tilde{u}^{c i}}, m_{\tilde{u}^{c j}}\right)\right] \\
& -\frac{\lambda_{X D}^{1} \lambda_{X D}^{2} \lambda_{D}^{i 1} \lambda_{D}^{i 2}}{8 \pi^{2}} m_{D}^{2} K\left(m_{D}, m_{D}, m_{\tilde{x}}, m_{\tilde{u}^{c i}}\right), \\
D_{R R}^{(D)}= & \frac{\lambda_{D}^{1 i} \lambda_{D}^{2 i} \lambda_{D}^{1 j} \lambda_{D}^{2 j}}{64 \pi^{2}}\left[2 H\left(m_{D}, m_{D}, m_{\tilde{d}^{c i}}, m_{\tilde{d}^{c j}}\right)\right. \\
& \left.+2 H\left(m_{d^{i}}, m_{d^{j}}, m_{\tilde{D}}, m_{\tilde{D}}\right)\right], \\
K_{L L}^{(D)}= & K_{L R}^{(D)}=D_{L L}^{(D)}=0 .
\end{aligned}
$$

\section{2. $\mu-e$ conversion}

Among the models under consideration, only the $X q \ell d^{c}$ type model is subject to the constraint from $\mu-e$ conversion $[48,49]$. As shown in Fig. (22), there are one-loop box diagrams contributing to $\mu-e$ conversion. These box diagrams can contribute only to the following current interactions:

$$
\begin{aligned}
\mathcal{L}_{\text {eff }}= & C_{L R}^{d}\left(\bar{e}_{L} \gamma^{\rho} \mu_{L}\right)\left(\bar{d}_{R} \gamma_{\rho} d_{R}\right)+C_{L L}^{d}\left(\bar{e}_{L} \gamma^{\rho} m u_{L}\right)\left(\bar{d}_{L} \gamma_{\rho} d_{L}\right) \\
& +C_{L L}^{u}\left(\bar{e}_{L} \gamma^{\rho} m u_{L}\right)\left(\bar{u}_{L} \gamma_{\rho} u_{L}\right),
\end{aligned}
$$

where $C_{P P^{\prime}}^{q}$ is the coefficient of the corresponding diagram. From the effective operators, we obtain the $\mu-e$ conversion branching ratio for ${ }_{13} \mathrm{Al}[50]$ :

$$
B_{\mu N \rightarrow e N}(Z=13) 2.0 \times \frac{1}{2 G_{F}^{2}}\left|2 C_{L L}^{u}+C_{L L}^{d}+C_{L R}^{d}\right|^{2},
$$

where $G_{F}$ is the Fermi constant.

For the model $(D)$,

$$
\begin{aligned}
C_{L R}^{d(D)}= & -\frac{\lambda_{D}^{i 1} \lambda_{D}^{i 2} \lambda_{X D}^{1} \lambda_{X D}^{2}}{32 \pi^{2}} m_{D}^{2} K\left(m_{D}, m_{D}, m_{\tilde{u}^{i}} m_{\tilde{x}}\right), \\
C_{L L}^{d(D)}= & \frac{\lambda_{D}^{i 1} \lambda_{D}^{i 2} \lambda_{D}^{1 j} \lambda_{D}^{1 j}}{64 \pi^{2}}\left[H\left(m_{u^{i}}, m_{\nu^{i}}, m_{\tilde{D}}, m_{\tilde{D}}\right)\right. \\
& \left.+H\left(m_{D}, m_{D}, m_{\tilde{u}^{i}}, m_{\tilde{\nu}^{i}}\right)\right], \\
C_{L L}^{u(D)}= & \frac{\lambda_{D}^{i 1} \lambda_{D}^{i 2} \lambda_{D}^{1 j} \lambda_{D}^{1 j}}{64 \pi^{2}}\left[H\left(m_{u^{i}}, m_{e^{i}}, m_{\tilde{D}}, m_{\tilde{D}}\right)\right. \\
& \left.+H\left(m_{D}, m_{D}, m_{\tilde{u}^{i}}, m_{\tilde{e}^{i}}\right)\right],
\end{aligned}
$$

where $i, j$ are flavor indices and the tilde over a particle name implies its supersymmetric scalar partner with odd $R$ parity. Note that we can safely ignore the masses of quarks and leptons except the top-quark mass, although we show generic results for the purpose of completeness.

Similarly, for models $(L)$ and $(Q)$ from Eqs. (2.3) and (2.4),

$$
\begin{aligned}
& C_{L R}^{d(L)}=-\frac{\lambda_{X L}^{1} \lambda_{X L}^{2} \lambda_{L}^{i 1} \lambda_{L}^{i 1}}{32 \pi^{2}} m_{L}^{2} K\left(m_{L}, m_{L}, m_{\tilde{x}}, m_{\tilde{u}^{i}}\right), \\
& C_{L L}^{d(L)}=0, \\
& C_{L L}^{u(L)}=-\frac{\lambda_{X L}^{1} \lambda_{X L}^{2} \lambda_{L}^{1 i} \lambda_{L}^{1 i}}{32 \pi^{2}} m_{L}^{2} K\left(m_{L}, m_{L}, m_{\tilde{x}}, m_{\tilde{d}^{c i}}\right),
\end{aligned}
$$

and

$$
\begin{aligned}
C_{L R}^{d(Q)}= & \frac{\lambda_{Q}^{1 i} \lambda_{Q}^{2 i} \lambda_{Q}^{j 1} \lambda_{Q}^{j 1}}{64 \pi^{2}}\left[H\left(m_{d^{i}}, m_{e^{i}}, m_{\tilde{Q}}, m_{\tilde{Q}}\right)\right. \\
& \left.+H\left(m_{Q}, m_{Q}, m_{\tilde{d}^{c i}}, m_{\tilde{e}^{i}}\right)\right], \\
C_{L L}^{d(Q)}= & 0, \\
C_{L L}^{u(Q)}= & -\frac{\lambda_{Q}^{1 i} \lambda_{Q}^{2 i} \lambda_{X Q}^{1} \lambda_{X Q}^{1}}{32 \pi^{2}} m_{Q}^{2} K\left(m_{Q}, m_{Q}, m_{\tilde{d}^{c i}}, m_{\tilde{x}}\right) .
\end{aligned}
$$




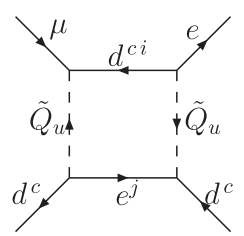

(a)

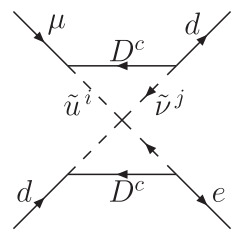

(f)

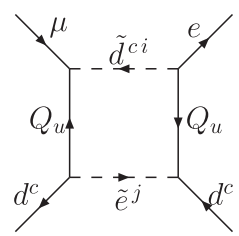

(b)

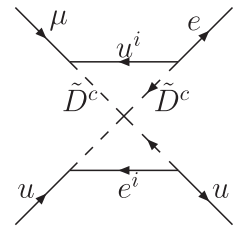

(g)

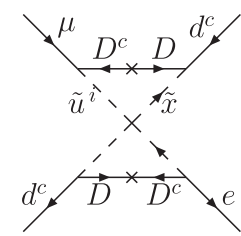

(c)

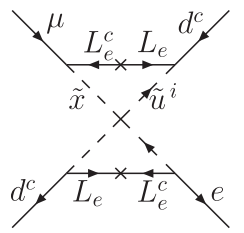

(d)

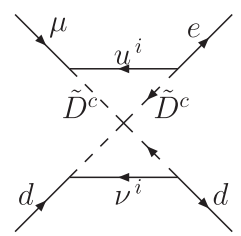

(e)

FIG. 22. One-loop box diagrams (a)-(j) contributing to $\mu-e$ conversion in $X q \ell d^{c}$ models. Here, we use two-component spinor notation.

\section{3. $\boldsymbol{B}_{s} \rightarrow \ell^{+} \ell^{-}, \boldsymbol{b} \rightarrow s \ell^{+} \ell^{-}$transition}

As with $\mu-e$ conversion described in the previous section, the $X q \ell d^{c}$ model is also subject to constraints from $b \rightarrow s$ transition measurements. At one-loop level, the contributing Feynman diagrams are listed in Figs. 4 and 23. The one-loop contributions lead to

$$
\begin{aligned}
\mathcal{L}_{\text {eff }}= & Z_{L L}\left(\bar{s}_{L} \gamma^{\rho} b_{L}\right)\left(\bar{\ell}_{L} \gamma_{\rho} \ell_{L}\right)+Z_{L R}\left(\bar{s}_{L} \gamma^{\rho} s_{L}\right)\left(\bar{\ell}_{R} \gamma_{\rho} \ell_{R}\right) \\
& +Z_{R L}\left(\bar{s}_{R} \gamma^{\rho} s_{R}\right)\left(\bar{\ell}_{L} \gamma_{\rho} \ell_{L}\right)+Z_{R R}\left(\bar{s}_{R} \gamma^{\rho} s_{R}\right)\left(\bar{\ell}_{R} \gamma_{\rho} \ell_{R}\right),
\end{aligned}
$$

where $\ell$ denotes the electron and muon. Note that there are no contributions to (scalar current)-(scalar current) interactions, such as $\bar{s}_{R} b_{L} \ell_{R} \ell_{L}$, since the UV completions of the $X q \ell d^{c}$ model involve only left-handed leptons. These induced effective couplings are constrained from various rare $B$-meson decays, for which the constraints on the scales of the effective operators are computed in [33].

We present the full one-loop $Z_{P P^{\prime}}$ from each UV completion $(D),(L)$ and $(Q)$. For the model $(D)$,

$Z_{L L}^{(D)}=\frac{\lambda_{D}^{3 i} \lambda_{D}^{2 i} \lambda_{D}^{j \ell} \lambda_{D}^{j \ell}}{64 \pi^{2}}\left[H\left(m_{\nu^{i}}, m_{u^{j}}, m_{\tilde{D}}, m_{\tilde{D}}\right)\right.$

$$
\left.+H\left(m_{D}, m_{D}, m_{\tilde{\nu}^{i}}, m_{\tilde{u}^{j}}\right)\right] \text {, }
$$

$Z_{R L}^{(D)}=-\frac{\lambda_{X D}^{2} \lambda_{X D}^{3} \lambda_{D}^{i \ell} \lambda_{D}^{i \ell}}{32 \pi^{2}} m_{D}^{2} K\left(m_{D}, m_{D}, m_{\tilde{x}}, m_{\tilde{u}^{i}}\right)$,

$Z_{L R}^{(D)}=Z_{R R}^{(D)}=0$,

where $\ell$ denotes the generation index of the external leptons, such that $\ell=1$ for the electron and 2 for the muon. For the $(L) \mathrm{UV}$ completion,
$Z_{R L}^{(L)}=-\frac{\lambda_{L}^{i 3} \lambda_{L}^{i 2} \lambda_{X L}^{\ell} \lambda_{X L}^{\ell}}{32 \pi^{2}} m_{L}^{2} K\left(m_{L}, m_{L}, m_{\tilde{u}^{i}}, m_{\tilde{x}}\right)$,

$Z_{L L}^{(L)}=Z_{L R}^{(L)}=Z_{R R}^{(L)}$.

For $(Q)$,

$$
\begin{aligned}
Z_{R L}^{(Q)}= & \frac{\lambda_{Q}^{i 3} \lambda_{Q}^{i 2} \lambda_{Q}^{\ell j} \lambda_{Q}^{\ell j}}{64 \pi^{2}}\left[H\left(m_{e^{i}}, m_{d^{j}}, m_{\tilde{Q}}, m_{\tilde{Q}}\right)\right. \\
& \left.+H\left(m_{Q}, m_{Q}, m_{\tilde{e}^{i}}, m_{\tilde{d}^{c j}}\right)\right], \\
Z_{L L}^{(Q)}= & Z_{L R}^{(Q)}=Z_{R R}^{(Q)} .
\end{aligned}
$$

We summarize the $b-s$ transition constraints in Table VII, assuming $m_{X} \sim m_{\tilde{x}} \ll m_{\text {soft }} \ll m_{D}, m_{L}, m_{Q}$, $m_{E}$, using the result from [33]. Since the constraints depend on whether the couplings are real or complex, we show both the strongest and weakest lower bounds in the table.

$$
\text { 4. } \boldsymbol{\mu}^{ \pm} \rightarrow \boldsymbol{e}^{ \pm} \boldsymbol{e}^{\mp} \boldsymbol{e}^{ \pm}
$$

For the $X q \ell d^{c}$ and $X \ell \ell e^{c}$ models, we have constraints from rare muon decays, with box diagrams contributing to $\mu \rightarrow 3 e$ decay. The relevant effective operators are

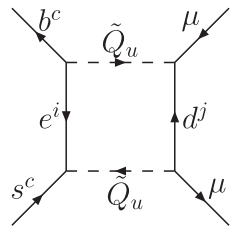

(a)

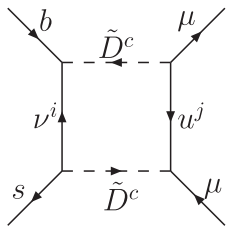

(b)

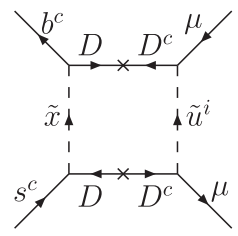

(c)
FIG. 23. Remaining box diagrams (a)-(c) that contribute to $B_{s} \rightarrow \mu^{+} \mu^{-}$, in addition to Fig. 4. Generic $b-s$ transitions can be easily deduced by replacing the external states in the diagrams. 
TABLE VII. Flavor constraints from the $b-s$ transition for the $X q \ell d^{c}$ models. The operator which is constrained is shown, along with the constraint on $\Lambda$ in TeV according to [33]. The numbers outside the parentheses are the strongest constraints and the numbers in the parentheses are the weakest constraints. For the UV completion model $\Phi, m_{\Phi} / \sqrt{\lambda^{2}}$ is constrained for tree level (boxed), and $m_{\Phi} / \sqrt{\lambda^{4}}$ is constrained for one-loop level (unboxed). Here, $R_{\Phi}=\log \left(m_{\Phi}^{2} / m_{\text {soft }}^{2}\right)-1 . i, j$ are flavor indices that run over 1,2 and 3 and the summation is implied, but $\ell$ denotes an electron or muon external state and thus is not summed over.

\begin{tabular}{|c|c|c|c|}
\hline \multirow{2}{*}{$\begin{array}{l}\text { Operator } \\
\text { Limit }(\mathrm{TeV})\end{array}$} & \multicolumn{3}{|c|}{$\lambda^{4}\left(\lambda^{2}\right.$ for tree level) in $X q \ell d^{c}$} \\
\hline & $D$ & $L$ & $Q$ \\
\hline$\overline{\left(\bar{s}_{L} \gamma^{\mu} b_{L}\right)\left(\bar{\ell}_{L} \gamma_{\mu} \ell_{L}\right)}$ & $\lambda_{D}^{3 \ell} \lambda_{D}^{2 \ell} \lambda_{D}^{3 i} \lambda_{D}^{2 i} \lambda_{D}^{j \ell} \lambda_{D}^{j \ell}$ & & $\lambda_{Q}^{i 3} \lambda_{Q}^{i 2} \lambda_{Q}^{\ell j} \lambda_{Q}^{\ell j}$ \\
\hline $45(16)$ & 32 (11) $2.5(0.9)$ & & $1.8(0.64)$ \\
\hline$\left(\bar{s}_{R} \gamma^{\mu} b_{R}\right)\left(\bar{\ell}_{L} \gamma_{\mu} \ell_{L}\right)$ & $\lambda_{X D}^{3} \lambda_{X D}^{2} \lambda_{D}^{i \ell} \lambda_{D}^{i \ell} R_{D}$ & $\lambda_{L}^{i 3} \lambda_{L}^{i 2} \lambda_{X L}^{\ell} \lambda_{X L}^{\ell} R_{L}$ & $\lambda_{Q}^{\ell 3} \lambda_{Q}^{2 \ell} \lambda_{Q}^{i 3} \lambda_{Q}^{i 2} \lambda_{Q}^{\ell j} \lambda_{Q}^{\ell j}$ \\
\hline $63(16)$ & $3.5(0.9)$ & $3.5(0.9)$ & 45 (11) $2.5(0.64)$ \\
\hline
\end{tabular}

$$
\begin{aligned}
\mathcal{L}_{\text {eff }}= & \frac{1}{2} A_{R R}\left(\bar{e}_{R} \gamma^{\rho} e_{R}\right)\left(\bar{e}_{R} \gamma_{\rho} \mu_{R}\right)+\frac{1}{2} A_{L L}\left(\bar{e}_{L} \gamma^{\rho} e_{L}\right)\left(\bar{e}_{L} \gamma_{\rho} \mu_{L}\right) \\
& +A_{R L}\left(\bar{e}_{R} \gamma^{\rho} e_{R}\right)\left(\bar{e}_{L} \gamma_{\rho} \mu_{L}\right)+A_{L R}\left(\bar{e}_{L} \gamma^{\rho} e_{L}\right)\left(\bar{e}_{R} \gamma_{\rho} \mu_{R}\right),
\end{aligned}
$$

where $A_{P P^{\prime}}$ 's $\left(P\right.$ and $P^{\prime}$ are $L$ or $R$ ) are the coefficients generated from the one-loop contribution. Note that we have symmetry factors for the RR and LL couplings.

The partial decay width of a muon to three electrons is

$$
\Gamma_{\mu \rightarrow e e e}=\frac{m_{\mu}^{5}}{3 \cdot 2^{11} \pi^{3}}\left(\left|A_{R R}\right|^{2}+\left|A_{L L}\right|^{2}+\left|A_{R L}\right|^{2}+\left|A_{L R}\right|^{2}\right)
$$

where $m_{\mu}$ is the muon mass. The branching fraction is currently constrained to be $\lesssim 10^{-12}$, with the total muon width being $\Gamma_{\mu}=G_{F}^{2} m_{\mu}^{5} /\left(192 \pi^{3}\right)$.

For the $X q \ell d^{c}$ operator, we have only a contribution to $A_{L L}$ since only left-handed leptons take part in the new physics couplings. We obtain the following effective operators for the UV completion models $(D),(L)$ and $(Q)$, respectively:

$$
\begin{aligned}
A_{L L}^{(D)}= & \frac{\lambda_{D}^{i 1} \lambda_{D}^{i 2} \lambda_{D}^{j 1} \lambda_{D}^{j 1}}{64 \pi^{2}}\left[2 H\left(m_{u^{i}}, m_{u_{j}}, m_{\tilde{D}}, m_{\tilde{D}}\right)\right. \\
& \left.+2 H\left(m_{D}, m_{D}, m_{\tilde{u}^{i}}, m_{\tilde{u}^{j}}\right)\right],
\end{aligned}
$$

$$
\begin{aligned}
A_{L L}^{(L)}= & \frac{\lambda_{X L}^{2}\left(\lambda_{X L}^{1}\right)^{3}}{64 \pi^{2}}\left[2 H\left(m_{X}, m_{X}, m_{\tilde{L}}, m_{\tilde{L}}\right)\right. \\
& \left.+2 H\left(m_{L}, m_{L}, m_{\tilde{x}}, m_{\tilde{x}}\right)\right],
\end{aligned}
$$

$$
\begin{aligned}
A_{L L}^{(Q)}= & \frac{\lambda_{Q}^{1 i} \lambda_{Q}^{2 i} \lambda_{Q}^{1 j} \lambda_{Q}^{1 j}}{64 \pi^{2}}\left[2 H\left(m_{Q}, m_{Q}, m_{\tilde{d}^{c i}}, m_{\tilde{d}^{c j}}\right)\right. \\
& \left.+2 H\left(m_{d^{i}}, m_{d^{j}}, m_{\tilde{Q}}, m_{\tilde{Q}}\right)\right] .
\end{aligned}
$$

Note that we generally have a factor of 2 larger contribution since the two electrons are identical.

We now summarize the results for $X \ell \ell e^{c}$ here. We have two UV completion models $(L)$ and $(E)$, as defined in Eqs. (2.13) and (2.14), respectively. For the model $(L)$, we have

$$
\begin{aligned}
A_{L L}^{(L)}= & \frac{1}{64 \pi^{2}}\left[\lambda _ { X L } ^ { 2 } ( \lambda _ { X L } ^ { 1 } ) ^ { 3 } \left[2 H\left(m_{X}, m_{X}, m_{\tilde{L}}, m_{\tilde{L}}\right)+2 H\left(m_{L}, m_{L}, m_{\tilde{x}}, m_{\tilde{x}}\right]+\lambda_{L}^{1 i} \lambda_{L}^{2 i} \lambda_{L}^{1 j} \lambda_{L}^{1 j}\left[2 H\left(m_{L}, m_{L}, m_{\tilde{e}^{c i}}, m_{\tilde{e}^{c j}}\right)\right.\right.\right. \\
& \left.\left.+2 H\left(m_{e^{i}}, m_{e^{j}}, m_{\tilde{L}}, m_{\tilde{L}}\right)\right]\right], \\
A_{R R}^{(L)}= & \frac{\lambda_{L}^{i 1} \lambda_{L}^{i 2} \lambda_{L}^{j 1} \lambda_{L}^{j 1}}{64 \pi^{2}}\left[2 H\left(m_{L}, m_{L}, m_{\tilde{e}^{i}}, m_{\tilde{e}^{j}}\right)+2 H\left(m_{e^{i}}, m_{e^{j}}, m_{\tilde{L}}, m_{\tilde{L}}\right)+2 H\left(m_{L}, m_{L}, m_{\tilde{\nu}^{i}}, m_{\tilde{\nu}^{j}}\right)+2 H\left(m_{\nu^{i}}, m_{\nu^{j}}, m_{\tilde{L}}, m_{\tilde{L}}\right)\right], \\
A_{R L}^{(L)}= & \frac{\lambda_{L}^{1 i} \lambda_{L}^{2 i} \lambda_{L}^{j 1} \lambda^{j 1}}{64 \pi^{2}}\left[H\left(m_{e^{i}}, m_{e^{j}}, m_{\tilde{L}}, m_{\tilde{L}}\right)+H\left(m_{L}, m_{L}, m_{\tilde{e}^{c i}}, m_{\tilde{e}^{j j}}\right)\right]-\frac{\lambda_{X L}^{1} \lambda_{X L}^{2} \lambda_{L}^{i 1} \lambda_{L}^{i 1}}{32 \pi^{2}} m_{L}^{2} K\left(m_{L}, m_{L}, m_{\tilde{x}}, m_{\tilde{\nu}^{i}}\right), \\
A_{L R}^{(L)}= & \frac{\lambda_{L}^{i 1} \lambda_{L}^{i 2} \lambda_{L}^{1 j} \lambda_{L}^{1 j}}{64 \pi^{2}}\left[H\left(m_{e^{i}}, m_{e^{j}}, m_{\tilde{L}}, m_{\tilde{L}}\right)+H\left(m_{L}, m_{L}, m_{\tilde{e}^{i}}, m_{\tilde{e}^{j}}\right)\right]-\frac{\lambda_{L}^{i 1} \lambda_{L}^{i 2} \lambda_{X L}^{1} \lambda_{X L}^{1}}{32 \pi^{2}} m_{L}^{2} K\left(m_{L}, m_{L}, m_{\tilde{\nu}^{i}}, m_{\tilde{x}}\right) .
\end{aligned}
$$

For the model $(E)$, 


$$
\begin{aligned}
A_{L L}^{(E)}= & \frac{\lambda_{E}^{13} \lambda_{E}^{23} \lambda_{E}^{1 j} \lambda_{E}^{1 j}}{64 \pi^{2}}\left[2 H\left(m_{\nu^{3}}, m_{\nu^{j}}, m_{\tilde{E}}, m_{\tilde{E}}\right)\right. \\
& \left.+2 H\left(m_{E}, m_{E}, m_{\tilde{\nu}^{3}}, m_{\tilde{\nu}^{i}}\right)\right], \\
A_{R R}^{(E)}= & \frac{\lambda_{X E}^{2}\left(\lambda_{X E}^{1}\right)^{3}}{64 \pi^{2}}\left(2 H\left(m_{X}, m_{X}, m_{\tilde{E}}, m_{\tilde{E}}\right)\right. \\
& \left.+2 H\left(m_{E}, m_{E}, m_{\tilde{x}}, m_{\tilde{x}}\right)\right), \\
A_{R L}^{(E)}= & -\frac{\lambda_{E}^{13} \lambda_{E}^{23} \lambda_{X E}^{1} \lambda_{X E}^{1}}{32 \pi^{2}} m_{E}^{2} K\left(m_{E}, m_{E}, m_{\tilde{x}}, m_{\tilde{\nu}^{3}}\right), \\
A_{L R}^{(E)}= & -\frac{\lambda_{X E}^{1} \lambda_{X E}^{2} \lambda_{E}^{1 j} \lambda_{E}^{1 j}}{32 \pi^{2}} m_{E}^{2} K\left(m_{E}, m_{E}, m_{\tilde{x}}, m_{\tilde{\nu}^{j}}\right),
\end{aligned}
$$

where the index $j$ runs over only the second and the third generation since the indices in $\lambda_{E}$ couplings are antisymmetric.

We summarize the $\mu^{-} \rightarrow e^{-} e^{+} e^{-}$constraints in Table VIII under the assumption $m_{X} \sim m_{\tilde{x}} \ll m_{\text {soft }} \ll$ $m_{D}, m_{L}, m_{Q}, m_{E}$. For the $X \ell \ell e^{c}$ model, the couplings in the table are

$$
\begin{aligned}
& {\left[\left(\lambda_{X L}^{2}\left(\lambda_{X L}^{1}\right)^{3}+\left(\lambda_{L}^{1 i} \lambda_{L}^{2 i}\right)^{2}\right)^{2}+4\left(\lambda_{L}^{i 1} \lambda_{L}^{i 2} \lambda_{L}^{j 1} \lambda_{L}^{j 1}\right)^{2}\right.} \\
& \quad+\frac{1}{4}\left(\lambda_{L}^{1 i} \lambda_{L}^{2 i} \lambda_{L}^{j 1} \lambda_{L}^{j 1}-\lambda_{X L}^{1} \lambda_{X L}^{2} \lambda_{L}^{i 1} \lambda_{L}^{i 1} R_{L}\right)^{2} \\
& \left.\quad+\frac{1}{4}\left(\lambda_{L}^{i 1} \lambda_{L}^{i 2} \lambda_{L}^{1 j} \lambda_{L}^{1 j}-\lambda_{L}^{i 1} \lambda_{L}^{i 2} \lambda_{X L}^{1} \lambda_{X L}^{1} R_{L}\right)^{2}\right]^{1 / 2}
\end{aligned}
$$

\begin{tabular}{|c|c|c|c|}
\hline \multicolumn{2}{|c|}{ Model } & Tree $\lambda^{2}$ & One loop $\lambda^{4}$ \\
\hline \multirow[t]{6}{*}{$X q \ell d^{c}$} & $D$ & & $\left(\lambda_{D}^{i 1} \lambda_{D}^{i 2}\right)^{2}$ \\
\hline & & & $9.8 \mathrm{TeV}$ \\
\hline & $L$ & & $\lambda_{X L}^{2}\left(\lambda_{X L}^{1}\right)^{3}$ \\
\hline & & & $9.8 \mathrm{TeV}$ \\
\hline & $Q$ & & $\left(\lambda_{Q}^{i 1} \lambda_{Q}^{2 i}\right)^{2}$ \\
\hline & & & $9.8 \mathrm{TeV}$ \\
\hline \multirow[t]{4}{*}{$X \ell \ell e^{c}$} & $L$ & $\lambda_{L}^{11} \sqrt{\left(\lambda_{L}^{12}\right)^{2}+\left(\lambda_{L}^{21}\right)^{2}}$ & Eq. (A35) \\
\hline & & $87 \mathrm{TeV}$ & $9.8 \mathrm{TeV}$ \\
\hline & $E$ & & Eq. (A36) \\
\hline & & & $9.8 \mathrm{TeV}$ \\
\hline
\end{tabular}

for the model $(L)$ and

TABLE VIII. Flavor constraints from $\mu^{-} \rightarrow e^{-} e^{+} e^{-}$. Each row represents the UV completion $\Phi$. The numbers below the couplings are the constraints $\Lambda$ in $\mathrm{TeV}$ on $m_{\Phi} / \sqrt{\lambda^{2}}$ for a treelevel contribution and $m_{\Phi} / \sqrt{\lambda^{4}}$ for a loop contribution.

$$
\begin{aligned}
& {\left[\left(\lambda_{E}^{13} \lambda_{E}^{23} \lambda_{E}^{1 j} \lambda_{E}^{1 j}\right)^{2}+\left(\lambda_{X E}^{2}\left(\lambda_{X E}^{1}\right)^{3}\right)\right)^{2}} \\
& \left.\quad+\frac{1}{4}\left(\left(\lambda_{E}^{13} \lambda_{E}^{23} \lambda_{X E}^{1} \lambda_{X E}^{1}\right)^{2}+\left(\lambda_{X E}^{1} \lambda_{X E}^{2} \lambda_{E}^{1 i} \lambda_{E}^{1 i}\right)^{2}\right) R_{E}^{2}\right]^{1 / 2}
\end{aligned}
$$

for the model $(E)$.

\section{APPENDIX B: DECAY THROUGH DIMENSION- FIVE EFFECTIVE OPERATORS}

This appendix summarizes the calculation of the LOSP decay width through dimension-five operators in the effective Lagrangian in ADM models. We consider three-body decays through a contact interaction and four-body decays through an off-shell intermediate particle as shown in Fig. 24.

The effective dimension-four superpotential operators of ADM models, which yield a dimension-five Lagrangian, are generically of the form

$$
W_{\mathrm{eff}}=\frac{d_{I J K L}}{\Lambda} \Phi_{1}^{I} \Phi_{2}^{J} \Phi_{3}^{L} \Phi_{4}^{L}=\frac{\lambda_{i j k l} c_{a b c d}}{\Lambda} \Phi_{1}^{i a} \Phi_{2}^{j b} \Phi_{3}^{k c} \Phi_{4}^{l d},
$$

where $I=(i, a), J=(j, b), K=(k, c)$ and $L=(l, d)$ represent the flavor indices $i, j, k, l$ and the gauge indices $a, b, c, d$, respectively, for the corresponding chiral superfields. ${ }^{8} d_{I J K L}$ 's are the coefficients of the superpotential term, which is factorized by a flavor-dependent coefficient $\lambda_{i j k l}$ and a purely gauge-group-dependent Clebsh-Gordon coefficient $c_{a b c d}$.

The three- and four-body decays of an $R$-parity odd scalar $\phi_{1}$ or an $R$-parity odd fermion $\psi_{0}$ are shown in Fig. 24 (which is identical to Fig. 1, though with the particles labeled now with numerical subscripts for notational clarity in what follows). We parametrize the ordinary MSSM interaction among $\psi_{0}, \varphi_{1}$ and $\psi_{5}$ by

$$
\begin{aligned}
\Delta L= & -\left(g_{1}\right)_{I J K} \phi_{1}^{I} \bar{\Psi}_{0}{ }^{J} P_{L} \Psi_{5}^{K} \\
& -\left(g_{2}\right)_{I J K} \phi_{2}^{I} \bar{\Psi}_{0}{ }^{J} P_{R} \Psi_{5}^{K}+\{\text { H.c. }\} \\
= & -\left(y_{1}\right)_{i j k} \tilde{c}_{a b c} \phi_{1}^{i a} \bar{\Psi}_{0}^{j b} P_{L} \Psi_{5}^{k c} \\
& -\left(y_{2}\right)_{i j k} \tilde{c}_{a b c} \phi_{2}^{i a} \bar{\Psi}_{0}{ }^{j b} P_{R} \Psi_{5}^{k c}+\{\text { H.c. }\}
\end{aligned}
$$

where $\psi_{3}, \psi_{4}$ and $\psi_{5}$ are SM fermions, and $g_{1}$ and $g_{2}$ are the coefficients. Here, we again use the collective notation for gauge and flavor indices with $I=(i, a), J=(j, b)$ and $K=(k, c) \cdot g_{1}$ and $g_{2}$ are factorized into flavor-dependent couplings $y_{1}$ and $y_{2}$, and a Clebsch-Gordon coefficient $\tilde{c}$ for

\footnotetext{
${ }^{8}$ In this generic calculation, we treat $\Phi_{1}, \Phi_{2}, \Phi_{3}$ and $\Phi_{4}$ as distinct fields. If some fields are the same, we need to compensate the resultant formulas by an appropriate symmetry factor in the definition of $W_{\text {eff }}$.
} 


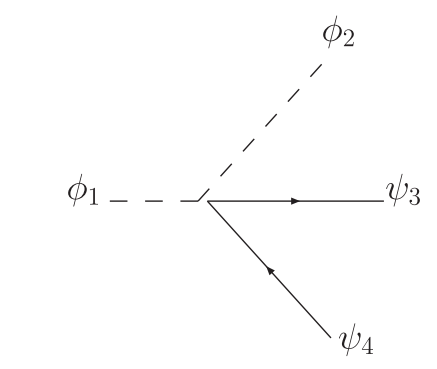

(a) Three-body decay

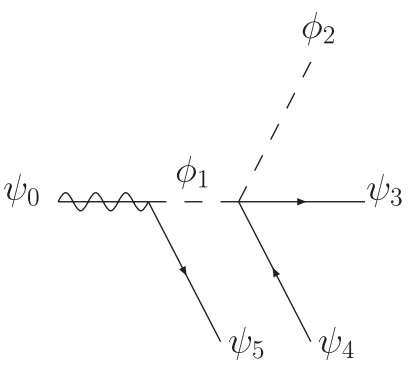

(b) Four-body decay
FIG. 24. General (a) three-body and (b) four-body decays of the LOSP in ADM models. Special cases are depicted in Fig. 1. Here, we denote scalar fields by $\phi$ and fermion fields by $\psi$.

the gauge group. We will take $\psi_{3}, \psi_{4}$ and $\psi_{5}$ to be massless since they are SM fermions, ignoring top quarks in the final state. $\phi_{2}$ will be the scalar particle of an ADM chiral multiplet $X$. In natural ADM scenarios, the mass of $\phi_{2}$ will be around $10 \mathrm{GeV}$. We can additionally simplify resultant expressions if we treat $\phi_{2}$ as being massless.

\section{Three-body decay through a contact interaction}

The spin-averaged amplitude square for the process of Fig. 24(a) is given by

$$
|\overline{\mathcal{M}}|^{2}=\frac{1}{N_{1}} \sum_{I, J, K, L} \frac{\left|d_{I J K L}\right|^{2}}{\Lambda^{2}}\left(4 p_{3} \cdot p_{4}\right),
$$

where $d_{I J K L}$ is the coefficient of the effective superpotential operator in Eq. (B1), and $I, J, K$ and $L$ denote the gauge and flavor indices collectively for $\phi_{1}, \phi_{2}, \psi_{3}$ and $\psi_{4}$, respectively. Here, the notation $p_{X}$ implies the momentum of particle $X$. We average the amplitude squared over the initial states of the decayed particle, so we have the number of internal degrees of freedom $N_{1}$ of $\phi_{1}$ in the denominator. For example, if $\phi_{1}$ is a color-triplet $S U(2)$-doublet scalar particle, $N_{1}$ is $3 \times 2=6$. Then, the differential decay width for the three-body decay process can be expressed in terms of invariant masses:

$$
d \Gamma=\frac{1}{(2 \pi)^{3}} \frac{1}{32 m_{1}^{3}}|\overline{\mathcal{M}}|^{2} d m_{23}^{2} d m_{34}^{2},
$$

where $m_{23}=\left(p_{2}+p_{3}\right)^{2}$ and $m_{34}=\left(p_{3}+p_{4}\right)^{2}$. The limits of integration for obtaining the total decay width are determined by the kinematic constraints on the system. In general cases with arbitrary masses, the integration domain is represented by a Dalitz plot. Considering only $m_{3}=m_{4}=0$, the domains for $m_{12}$ and $m_{34}$ are given by

$$
\left(m_{23}^{2}\right)_{\min }=m_{2}^{2}, \quad\left(m_{23}^{2}\right)_{\max }=m_{1}^{2},
$$

$$
\left(m_{34}^{2}\right)_{\min }=0, \quad\left(m_{34}^{2}\right)_{\max }=\frac{\left(m_{23}^{2}-m_{2}^{2}\right)\left(m_{1}^{2}-m_{23}^{2}\right)}{m_{23}^{2}} .
$$

By integrating over the domain, we obtain the total decay width

$$
\begin{aligned}
\Gamma= & \frac{C_{S U(2)} C_{S U(3)}}{\left(128 \pi^{3} N_{1}\right)\left(m_{1}^{3} \Lambda^{2}\right)}\left(\sum_{i, j, k, l}\left|\lambda_{i j k l}\right|^{2}\right) \\
& \times\left[\frac{1}{6}\left(m_{1}^{2}-m_{2}^{2}\right)\left(m_{1}^{4}+10 m_{1}^{2} m_{2}^{2}+m_{2}^{4}\right)-m_{1}^{2} m_{2}^{2}\left(m_{1}^{2}+m_{2}^{2}\right)\right. \\
& \left.\times \log \left(m_{1}^{2} / m_{2}^{2}\right)\right] .
\end{aligned}
$$

Note that we factorize the coupling factor $\sum_{i, j, k, l}\left|d_{i j k l}\right|^{2}$ into the flavor-dependent coupling squared $\left(\sum_{i, j, k, l}\left|\lambda_{i j k l}\right|^{2}\right)$ and the group theoretical factor $C_{S U(2)} C_{S U(3)}$, assuming only $S U(2)$ and $S U(3)$ groups are relevant. In Table IX, we summarize $C_{S U(2)}$ and $C_{S U(3)}$ for various possible combinations of the representations of participating particles.

\section{Four-body decay through an intermediate off-shell particle}

Next we consider the case of Fig. 24(b). The spinaveraged amplitude squared is

$$
\begin{aligned}
|\overline{\mathcal{M}}|^{2}= & \frac{1}{2 N_{0}} \sum_{I, I^{\prime}, J, K, L, M, N}\left[\left(g_{1}^{*} g_{1}^{\prime}+g_{2}^{*} g_{2}^{\prime}\right) \frac{d d^{\prime *}}{\Lambda^{2}}\right]\left(\frac{1}{q^{2}-m_{1}^{2}}\right)^{2} \\
& \times\left(4 p_{0} \cdot p_{5}\right)\left(4 p_{3} \cdot p_{4}\right),
\end{aligned}
$$

TABLE IX. Group theoretical factors for three-body decay through a contact interaction. Here, $\delta_{b}^{a}$ is the Kronecker delta, and $\epsilon_{a b}$ and $\epsilon_{a b c}$ are Levi-Civita symbols. Note that the overall switch between complex representations 3 and $\overline{3}$ does not change the factor.

\begin{tabular}{cccc}
\hline \hline$\left(R_{1}, R_{2}, R_{3}, R_{4}\right)^{S U(2)}$ & $C_{S U(2)}$ & $\left(R_{1}, R_{2}, R_{3}, R_{4}\right)^{S U(3)}$ & $C_{S U(3)}$ \\
\hline$(1,1,1,1)$ & 1 & $(1,1,1,1)$ & 1 \\
$(1,1,2,2) \&$ perm & $\epsilon_{a b} \epsilon^{a b}=2$ & $(3, \overline{3}, 1,1) \&$ perm & $\delta_{a}^{b} \delta_{b}^{a}=3$ \\
$(2,2,2,2) \&$ perm & $\epsilon_{a b} \epsilon_{c d} \epsilon^{a b} \epsilon^{c d}=4$ & $(3, \overline{3}, 3, \overline{3}) \&$ perm & $\delta_{a}^{b} \delta_{c}^{d} \delta_{b}^{a} \delta_{d}^{c}=9$ \\
& & $(3,3,3,1) \&$ perm & $\epsilon_{a b c} \epsilon^{a b c}=6$ \\
\hline \hline
\end{tabular}


TABLE X. Group theoretical factors for four-body decay through an intermediate (off-shell) particle. $\left(T^{A}\right)_{a b}$ is $\sigma_{a b}^{A} / 2$ for $S U(2)$ and $\lambda_{a b}^{A} / 2$ for $S U(3)$, where $\sigma^{a}$ 's and $\lambda^{a}$ 's are Pauli and Gell-Mann matrices, respectively. Permutations are defined only within underlined items (if the underline is disconnected, they are two separate permutation sets). Switching 3 with $\overline{3}$ altogether leads to the same factor.

\begin{tabular}{|c|c|c|c|}
\hline \multicolumn{2}{|c|}{$S U(2)$} & \multicolumn{2}{|c|}{$S U(3)$} \\
\hline$\left(R_{0}, R_{5}, R_{1}, R_{2}, R_{3}, R_{4}\right)$ & $D_{S U(2)}$ & $\left(R_{0}, R_{5}, R_{1}, R_{2}, R_{3}, R_{4}\right)$ & $D_{S U(3)}$ \\
\hline$(1,1,1,1,1,1)$ & 1 & $(1,1,1,1,1,1)$ & 1 \\
\hline$(1,1,1,2,2,1) \&$ perm & $\epsilon_{b c} \epsilon^{b c}=2$ & $(1,1,1,3, \overline{3}, 1) \&$ perm & $\delta_{b}^{c} \delta_{c}^{b}=3$ \\
\hline$(2,2,1,1,1,1)$ & $\epsilon_{e f} \epsilon^{e f}=2$ & $(1,1,1,3,3,3)$ & $\epsilon_{b c d} \epsilon_{b c d}=6$ \\
\hline$(2,2,1,2,2,1) \&$ perm & $\epsilon_{e f} \epsilon^{e f} \epsilon_{b c} \epsilon^{b c}=4$ & $(3, \overline{3}, 1,1,1,1)$ & $\delta_{e}^{f} \delta_{f}^{e}=3$ \\
\hline$(2,1,2,2,1,1) \&$ perm & $\epsilon_{f a} \epsilon^{f a^{\prime}} \epsilon^{a b} \epsilon_{a^{\prime} b}=2$ & $(3, \overline{3}, 1,3, \overline{3}, 1) \&$ perm & $\delta_{e}^{f} \delta_{f}^{e} \delta_{b}^{c} \delta_{c}^{b}=9$ \\
\hline$(2,1,2,2,2,2) \&$ perm & $\epsilon_{f a} \epsilon^{f a^{\prime}} \epsilon^{a b} \epsilon^{c d} \epsilon_{a^{\prime} b} \epsilon_{c d}=4$ & $(3, \overline{3}, 1,3,3,3)$ & $\delta_{e}^{f} \delta_{f}^{e} \epsilon_{b c d} \epsilon^{b c d}=18$ \\
\hline$(3, \overline{2}, 2,2,1,1) \&$ perm & $\left(T^{e}\right)_{a}^{f}\left(T^{e}\right)_{f}^{a^{\prime}} \epsilon^{a b} \epsilon_{a^{\prime} b}=\frac{3}{2}$ & $(3,1, \overline{3}, 3,1,1) \&$ perm & $\delta_{f}^{a} \delta_{a^{\prime}}^{; f} \delta_{a}^{b} \delta_{b}^{a^{\prime}}=3$ \\
\hline$(3, \overline{2}, 2,2, \overline{2}, 2) \&$ perm & $\left(T^{e}\right)_{a}^{f}\left(T^{e}\right)_{f}^{a^{\prime}} \times$ & $(3,1, \overline{3}, \overline{3}, \overline{\overline{3}}, 1) \&$ perm & $\delta_{f}^{a} \delta_{a^{\prime}}^{f} \epsilon_{a b c} \epsilon^{a^{\prime} b c}=6$ \\
\hline & $\times \epsilon^{a b} \epsilon^{c d} \epsilon_{a^{\prime} b} \epsilon_{c d}=3$ & $(3,1, \overline{3}, 3, \overline{3}, 3) \&$ perm & $\delta_{f}^{a} \delta_{a^{\prime}}^{f} \delta_{a}^{b} \delta_{c}^{d} \delta_{b}^{a^{\prime}} \delta_{d}^{c}=9$ \\
\hline & & $(\overline{3}, \overline{3}, \overline{3}, 3,1,1) \&$ perm & $\epsilon^{f e a} \epsilon_{f e a^{\prime}} \delta_{a}^{b} \delta_{b}^{a^{\prime}}=6$ \\
\hline & & $(\overline{3}, \overline{3}, \overline{3}, \overline{3}, \overline{\overline{3}}, 1) \&$ perm & $\epsilon^{f e a} \epsilon_{f e a^{\prime}} \epsilon_{a b c} \epsilon^{a^{\prime} b c}=12$ \\
\hline & & $(\overline{3}, \overline{3}, \overline{3}, 3, \overline{3}, 3) \&$ perm & $\epsilon^{f e a} \epsilon_{f e a^{\prime}} \delta_{a}^{b} \delta_{c}^{d} \delta_{b}^{a^{\prime}} \delta_{d}^{c}=18$ \\
\hline & & $(8,3, \overline{3}, 3,1,1) \&$ perm & $\left(T^{e}\right)_{a}^{f}\left(T^{e}\right)_{f}^{a^{\prime}} \delta_{b}^{a} \delta_{a^{\prime}}^{b}=4$ \\
\hline & & $(8,3, \overline{3}, \overline{3}, \overline{\overline{3}}, 1) \&$ perm & $\left(T^{e}\right)_{a}^{f}\left(T^{e}\right)_{f}^{a^{\prime}} \epsilon^{a b c} \epsilon_{a^{\prime} b c}=8$ \\
\hline & & $(8,3, \overline{3}, 3, \overline{\overline{3}}, 3) \&$ perm & $\left(T^{e}\right)_{a}^{f}\left(T^{e}\right)_{f}^{a^{\prime}} \delta_{b}^{a} \delta_{c}^{d} \delta_{a^{\prime}}^{b} \delta_{d}^{c}=12$ \\
\hline
\end{tabular}

where the summation over $I=(i, a)$ and $I^{\prime}=\left(i^{\prime}, a^{\prime}\right)$ is from the intermediate $\phi_{1}$ exchange, and $J=(j, b)$, $K=(k, c), L=(l, d), M=(m, e), N=(n, f)$ denote the collective flavor indices $i, j, \ldots$ and gauge indices $a, b, \ldots$ of the external particles $\phi_{2}, \psi_{3}, \psi_{4}, \psi_{0}$ and $\psi_{5}$, respectively. $q=p_{2}+p_{3}+p_{4}$ is the momentum of the intermediate $\phi_{1}$. Here, we use abbreviations $g_{1}=\left(g_{1}\right)_{I M N}$, $g_{1}^{\prime}=\left(g_{1}\right)_{I^{\prime} M N}$ (and $g_{2}$ and $g_{2}^{\prime}$ in the same way), $d=d_{I J K L}$ and $d^{\prime}=d_{I^{\prime} J K L}$, where $d$ 's and $g$ 's are defined in Eqs. (B1) and (B2). $N_{0}$ represents the number of internal degrees of freedom of $\psi_{0}$, as in the three-body decay case.

With similar tricks to the three-body decay case by using the invariant masses of subsystems as integration variables and decomposing the four-body phase space (PS) integration into the three-body and the two-body PS integrations, one can get the analytic formula for the full decay width in the following integral form:

$$
\begin{aligned}
\Gamma= & \frac{1}{3 \cdot 2^{10} \pi^{5} N_{0}} \frac{1}{\Lambda^{2} m_{0}^{3}}\left(\sum_{I, I^{\prime}, J, K, L, M, N}\left(g_{1}^{*} g_{1}^{\prime}+g_{2}^{*} g_{2}^{\prime}\right)\left(d d^{*}\right)\right) \\
& \times \int_{m_{2}^{2}}^{m_{0}^{2}} d q^{2} \frac{\left(m_{0}^{2}-q^{2}\right)^{2}}{\left(q^{2}-m_{1}^{2}\right)^{2}} \frac{1}{q^{2}}\left[\frac { 1 } { 6 } ( q ^ { 2 } - m _ { 2 } ^ { 2 } ) \left(q^{4}+10 q^{2} m_{2}^{2}\right.\right. \\
& \left.\left.+m_{2}^{4}\right)-q^{2} m_{2}^{2}\left(q^{2}+m_{2}^{2}\right) \log \left(q^{2} / m_{2}^{2}\right)\right] .
\end{aligned}
$$

For nonzero $m_{2}$, the analytic result from the integration in Eq. (B9) is rather complicated. For the most nondegenerate cases where the SUSY particle mass difference is larger than the typical ADM mass $(\sim 10 \mathrm{GeV})$, we can safely assume that $m_{2}=0$. In such cases, the total decay width has a simplified form:

$$
\begin{aligned}
\Gamma= & \frac{D_{S U(2)} D_{S U(3)}}{3 \cdot 2^{10} \pi^{5} N_{0}} \frac{1}{\Lambda^{2} m_{0}^{3}}\left[\sum _ { i , i ^ { \prime } , j , k , l , m , n } \left(\left(y_{1}^{*}\right)_{i m n}\left(y_{1}\right)_{i^{\prime} m n}\right.\right. \\
& \left.\left.+\left(y_{2}^{*}\right)_{i m n}\left(y_{2}\right)_{i^{\prime} m n}\right)\left(\lambda_{i j k l} \lambda_{i^{\prime} j k l}^{*}\right)\right] \\
& \times\left[\frac{1}{3} m_{0}^{2}\left(m_{0}^{4}-12 m_{0}^{2} m_{1}^{2}+12 m_{1}^{4}\right)\right. \\
& \left.+2 m_{1}^{2}\left(m_{1}^{2}-m_{0}^{2}\right)\left(2 m_{1}^{2}-m_{0}^{2}\right) \log \left(\frac{m_{1}^{2}-m_{0}^{2}}{m_{1}^{2}}\right)\right],
\end{aligned}
$$

where we factorize the coupling factor $\sum\left(g_{1}^{*} g_{1}^{\prime}+\right.$ $\left.g_{2}^{*} g_{2}^{\prime}\right)\left(d d^{*}\right)$ into the flavor-dependent coupling squared in terms of $y_{1}$ and $y_{2}$ defined in Eq. (B2) with explicit flavor indices and the group theoretical factor $D_{S U(2)} D_{S U(3)}$, assuming only $S U(2)$ and $S U(3)$ groups are relevant again. We summarize $D_{S U(2)}$ and $D_{S U(3)}$ for various combinations in Table $\mathrm{X}$. One can check that $\Gamma \sim \frac{m_{0}^{7}}{\Lambda^{2} m_{1}^{4}}$ in the limit $m_{1} \gg m_{0}$, as expected from a simple dimensional argument.

\section{APPENDIX C: THE ATLAS ANALYSIS OBSERVABLES}

We summarize the experimental observables used for the ATLAS 0-lepton + 2-6-jet + MET and 1-2-lepton + 3-6-jet + MET analyses in the following. 
(i) $\vec{p}_{T}^{\text {miss: }}$ : Missing transverse momentum. The negative vector sum of the transverse momentum $p_{T}$ 's of identifiable objects.

(ii) $E_{T}^{\text {miss }}=\mid \vec{p}_{T}^{\text {miss }}$ : Missing transverse energy.

(iii) $\vec{p}_{T}\left(j_{i}\right)$ : The transverse momentum $\vec{p}_{T}$ of the $i$ th hardest jet in $p_{T}$ size ordering. Without $\rightarrow$, it implies the magnitude.

(iv) $\Delta \phi\left(\mathrm{obj}, E_{T}^{\text {miss }}\right)$ : Azimuthal angle between $\vec{p}_{T}$ of a given object (jet or lepton) and $\vec{p}_{T}^{\text {miss }}$.

(v) $m_{\text {eff }}(n j)$ : Effective mass with the hardest $n$ jets in $p_{T}$ size ordering. $m_{\mathrm{eff}}(n j)=\sum_{\ell} p_{T}(\ell)+$ $\sum_{i=1 \ldots, n} p_{T}\left(j_{i}\right)+E_{T}^{\text {miss }}$ including all signal leptons. In the 0-lepton analysis, $m_{\text {eff }}(N j)$ means $N=$ 2, 3, 4, 5, 6 for channels A, B, C, D and E, respectively. In the 1-2-lepton analysis, $m_{\mathrm{eff}}^{\text {excl. }}$ is defined similarly.

(vi) $m_{\text {eff }}$ (incl.): Inclusive effective mass. Effective mass defined with all jets with $p_{T}>40 \mathrm{GeV}$ for the 0-lepton analysis, or with all signal jets for the 1lepton analysis.

(vii) $N_{b-\text { tag }}$ : Number of $b$-tagged jets.

(viii) $m_{T}$ : Transverse mass of the lepton $\ell$ (for a single lepton event) and $\vec{p}_{T}^{\text {miss }}, \quad m_{T}=$ $\sqrt{2 p_{T}^{\ell} E_{T}^{\text {miss }}\left[1-\cos \Delta \phi\left(\ell, E_{T}^{\text {miss }}\right)\right]}$.

(ix) $\Delta R_{\min }($ jet, $\ell)$ : The minimum of $\Delta R=\sqrt{\Delta \eta^{2}+\Delta \phi^{2}}$ between the lepton $\ell$ (for a single lepton event) and each signal jet.

(x) $\Delta \phi_{\min }=\min \left(\Delta \phi\left(j_{1}, E_{T}^{\text {miss }}\right), \Delta \phi\left(j_{2}, E_{T}^{\text {miss }}\right)\right)$, where $j_{1}$ and $j_{2}$ are the first and second hardest jets, respectively.

(xi) $m_{C T}$ : Contransverse mass of the two $b$ jets (for two $b$-jet events) defined by $m_{C T}^{2}\left(b_{1}, b_{2}\right)=\left[E_{T}^{b_{1}}+E_{T}^{b_{2}}\right]^{2}-$ $\left[\vec{p}_{T}^{b_{1}}-\vec{p}_{T}^{b_{2}}\right]^{2}$.

\section{APPENDIX D: EVCHAIN, SUBPROCESS CHAINING FOR EVENT GENERATION}

The event generation that is required for the analyses we have carried out in this paper has a few technical challenges. As we see in Figs. 9 and 10, the cascade decay from the gluino and/or the squarks gives rise to a large number of outgoing particles at parton level, so the phase space becomes very high dimensional. In addition, the LOSP decays through a nonrenormalizable interaction, and in particular, the neutralino LOSP leads to a four-body decay through an intermediate off-shell squark or slepton. We also have exotic color vertices that involve color index contraction with the invariant tensor $\epsilon^{i j k}(i, j, k$ 's are color indices) in the case of the $u^{c} d^{c} d^{c}$ models.

Such technical challenges strongly restrict the choice of available tools. As of now, nonrenormalizable interactions and exotic color vertices can be treated successfully by using MADGRAPH5 [41]. However, MADGRAPH5 generates events by a Monte Carlo integration of the matrix element over the full phase space, and with a higher multiplicity of final state particles, the integration often leads to unbearably slow performance and a big accumulation of error. This problem becomes worse if we have several on-shell particles in the process with narrow decay widths since more careful sampling near on-shell poles is needed for a given required accuracy, which will take more sampling iterations and will thus be more prone to numerical errors.

Therefore, it is much more desirable to generate events by splitting a process into a few subprocesses-production channels and decay modes-and to connect the subprocesses into a single big event by making an appropriate transformation. Many Monte Carlo event generators indeed do the job in this way. For MADGRAPH5, an external tool called BRIDGE is designed to address this issue [51]. However, as far as we know, the BRIDGE tool is restricted to two-body or three-body decays for each decay subprocess, and it is not clear whether the tool has been actively maintained with the recent rapid changes of MadGraph5.

We address this difficulty by creating our own in-house tool called EVCHAIN [44]: an event chaining tool that automatically orders MADGRAPH5 event generation for each subprocess and combines resultant Les Houches Event (LHE) format files [52] into a single LHE format file by making appropriate Lorentz transformations and color flow number adjustments. Although the current version is tightly incorporated with MADGRAPH5, the general idea of EVCHAIN is not restricted to MADGRAPH5 since we treat each subprocess as a module with an interface of incoming and outgoing particles. Insofar as incoming and outgoing particle types are matched, any event generator with any specific process can be used for generating each subprocess. We also note that we do not aim to provide an automatic decay width calculation, differently from BRIDGE. The total decay width must be provided by MADGRAPH5 or the equivalent, while a relative branching ratio in one specific subprocess is automatically given by actual event generation. By this design choice, we simplified program requirements and we were able to generalize easily to any $\mathrm{N}$-body decay processes. In the following, we describe the tool in more detail.

EVCHAIN works as a "meta-event-generator" that supervises MADGRAPH5 event generation for subprocesses. In Fig. 25, we show the overall pipeline of EVCHAIN event generation. The tool is written in haskell [53]. Haskell is a pure functional programming language which excels in symbolic computation and many parsing tasks. The program is buildable by using the Glasgow Haskell Compiler GHC 7.4 or higher [54]. Currently, EVCHAIN is a software library, and a user needs to create a program using the EVCHAIN library for event generation.

In the source code of the user's program, the total event process is specified as a haskell tree data structure. The specification language as an embedded domain specific 


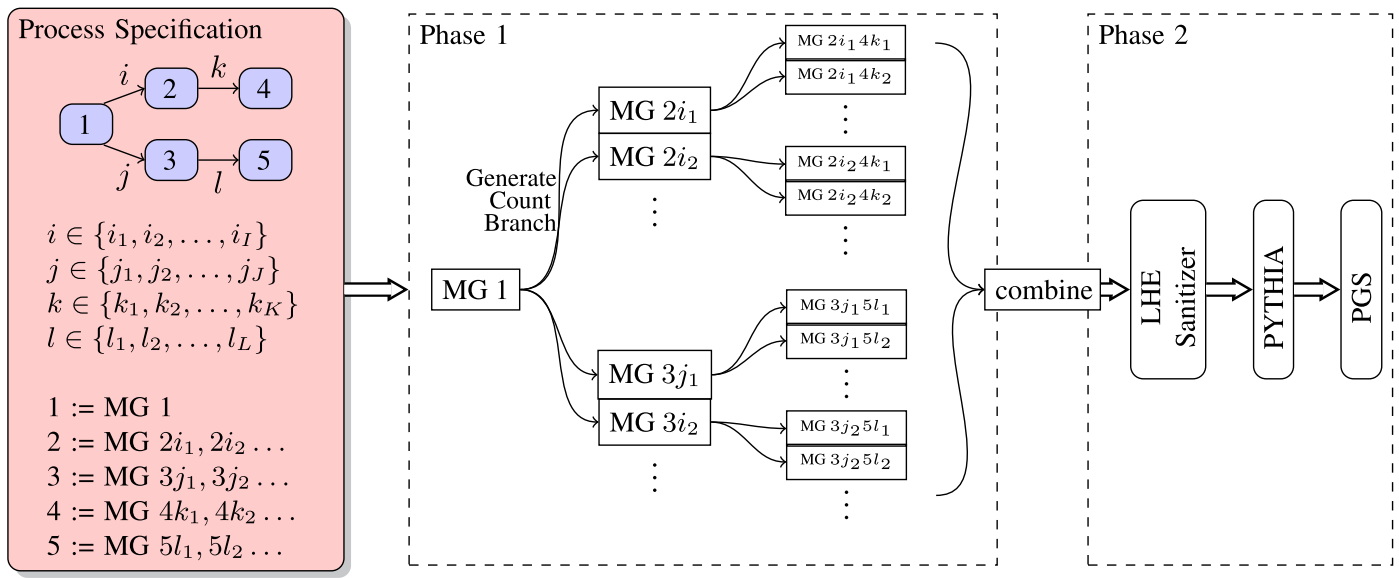

FIG. 25 (color online). The EVCHAIN pipeline.

language (EDSL) for EVCHAIN inside the haskell program is self-explanatory. We provide one example of such a specification description in Fig. 26, which is gluino pair production of the $q \ell d^{c}$ model with neutralino LOSP as shown in Fig. 26. A total process is a production process module with two incoming particles and an arbitrary number of outgoing particles. Each outgoing particle can be either a terminal particle or a decay process, which is a module with one incoming particle and an arbitrary number of outgoing particles, where again an outgoing particle of a decay process is either a terminal particle or a decay process, recursively. An incoming/terminal particle is specified by a list of PDG codes, so we can define a collection of particles as incoming or outgoing particles for convenience. Each subprocess is mapped into the MADGRAPH5 processes. In the example, the total process is defined in TOTAL_PROCESS, which has DECAY_GLUINO, and DECAY_GLUINO is again defined by DECAY_ NEUTRALINO. MADGRAPH_PROCESS_MAP defines actual MADGRAPH5 commands for each subprocess.

When running, the program will first prepare MADGRAPH5 directories for each subprocess. As shown in Fig. 25, the on-shell particles (denoted as $i, j, k, l$ in the figure) that connect mother and daughter subprocesses can

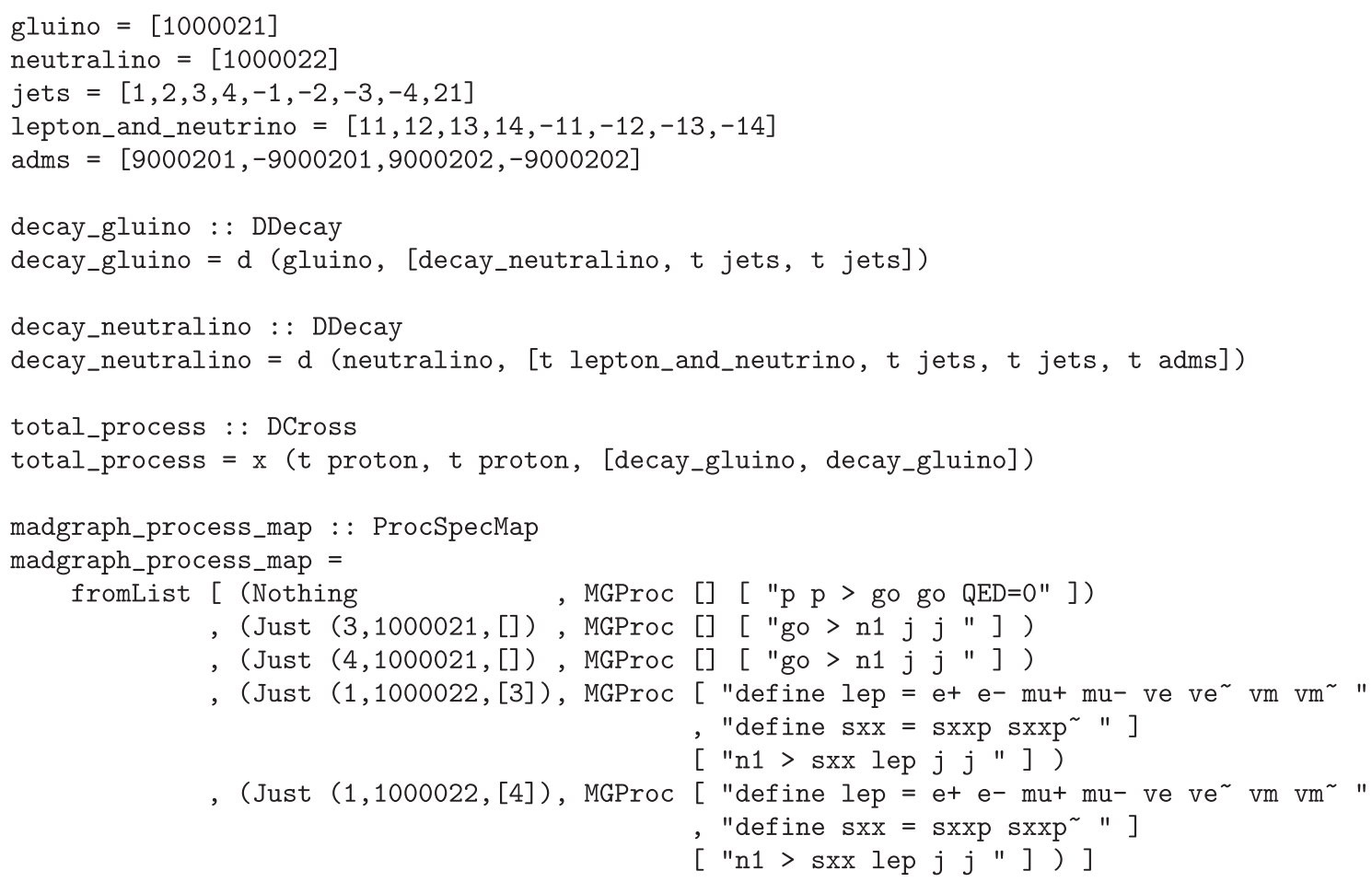

FIG. 26. A haskell code example of evchain process specification for the $\tilde{g}-\tilde{g}$ production of the $q \ell d^{c}$ model with neutralino LOSP as shown in Fig. 10(a). Note that we define the multiparticles JETS, LEPTON_AND_NEUTRINO and ADMS for the sake of convenience. 
be multiple particles. evchain automatically prepares for all of the cases as different working directories and avoids a name clash by making different hash numbers for distinct subprocesses and particles. Since the same hash number is produced for the same process specification, the preparation step can be efficiently done only once for repeating event generations with different parameter sets. evchain provides a configuration method for customizing the directory paths of relevant tools and working directories, which is adjustable for various cluster computing setups.

After the preparation step, the event generation is done in two stages: (i) generating LHE event files for each subprocess in the order of subprocess dependency and (ii) combining LHE event files into a single LHE file to pass to the rest of the event generator (event file sanitization, parton shower and hadronization using PYTHIA, and detector simulation using PGS). EVCHAIN facilitates an event counter and classifier. In every step after finishing each subprocess event generation, EVCHAIN counts the number of outgoing particles and orders the next dependent subprocess event generation for only the required number as determined by the previous step. Once all of the subprocess event generation is done, the combining routine runs through all events of the root subprocess and recursively finds events in daughter subprocesses and chains them by adjusting particle numbers and color flow numbers and transforming particle momenta in the daughter process from the rest frame of the decayed particle to the mother frame. After this, the total number of events of the resultant LHE file are automatically matched with the number the user specifies, and the LHE file is fed into the rest of the pipeline.

The EVCHAIN tool is designed to fit as a subsystem of PIPELINE, a cluster job coordinator for common high-energy physics tasks, described in Appendix B. 3 in [55]. We plan to improve the system with better support for general cluster facilities, at the same time as implementing missing functionality such as spin correlations.
[1] S. Nussinov, Phys. Lett. 165B, 55 (1985).

[2] G. B. Gelmini, L. J. Hall, and M. J. Lin, Nucl. Phys. B281, 726 (1987).

[3] S. M. Barr, R. S. Chivukula, and E. Farhi, Phys. Lett. B 241, 387 (1990).

[4] W. A. Bardeen, J. Kodaira, and T. Muta, Electroweak symmetry breaking. Proceedings, International Workshop, Hiroshima, Japan, November 12-15, 1991 (World Scientific, Singapore, Singapore, 1992), p. 451.

[5] S. B. Gudnason, C. Kouvaris, and F. Sannino, Phys. Rev. D 74, 095008 (2006).

[6] M. J. Strassler and K. M. Zurek, Phys. Lett. B 651, 374 (2007).

[7] D. E. Kaplan, M. A. Luty, and K. M. Zurek, Phys. Rev. D 79, 115016 (2009).

[8] K. M. Zurek, arXiv:1308.0338.

[9] M. J. Strassler, arXiv:hep-ph/0607160.

[10] D. Hooper and K. M. Zurek, Phys. Rev. D 77, 087302 (2008).

[11] P. W. Graham, D. E. Kaplan, S. Rajendran, and P. Saraswat, J. High Energy Phys. 07 (2012) 149.

[12] J. Fan, M. Reece, and J. T. Ruderman, J. High Energy Phys. 11 (2011) 012.

[13] J. Fan, M. Reece, and J. T. Ruderman, J. High Energy Phys. 07 (2012) 196.

[14] F. Zwirner, Phys. Lett. 132B, 103 (1983).

[15] H. Davoudiasl, D. E. Morrissey, K. Sigurdson, and S. Tulin, Phys. Rev. Lett. 105, 211304 (2010).

[16] H. Davoudiasl, D. E. Morrissey, K. Sigurdson, and S. Tulin, Phys. Rev. D 84, 096008 (2011).

[17] S. Chang and M. A. Luty, arXiv:0906.5013.

[18] C. Csaki, Y. Grossman, and B. Heidenreich, Phys. Rev. D 85, 095009 (2012).
[19] J. T. Ruderman, T. R. Slatyer, and N. Weiner, J. High Energy Phys. 09 (2013) 094.

[20] J. Kile and A. Soni, Phys. Rev. D 84, 035016 (2011).

[21] J. F. Kamenik and J. Zupan, Phys. Rev. D 84, 111502 (2011).

[22] P. Agrawal, S. Blanchet, Z. Chacko, and C. Kilic, Phys. Rev. D 86, 055002 (2012).

[23] A. Kumar and S. Tulin, Phys. Rev. D 87, 095006 (2013).

[24] J. Kile, arXiv:1308.0584.

[25] G. Isidori, Y. Nir, and G. Perez, Annu. Rev. Nucl. Part. Sci. 60, 355 (2010).

[26] ATLAS Collaboration, Report No. ATLAS-CONF-2012-061.

[27] G. Aad et al. (ATLAS Collaboration), Phys. Lett. B 713, 387 (2012).

[28] S. Chatrchyan et al. (CMS Collaboration), J. High Energy Phys. 04 (2012) 033.

[29] R. Aaij et al. (LHCb Collaboration), Phys. Rev. Lett. 108, 231801 (2012).

[30] RAaij et al. (LHCb Collaboration), Phys. Rev. Lett. 110, 021801 (2013).

[31] RAaij et al. (LHCb Collaboration), Phys. Rev. Lett. 111, 101805 (2013).

[32] S. Chatrchyan et al. (CMS Collaboration), Phys. Rev. Lett. 111, 101804 (2013).

[33] W. Altmannshofer and D. M. Straub, J. High Energy Phys. 08 (2012) 121.

[34] ATLAS Collaboration, Report No. ATLAS-CONF-2013-047.

[35] ATLAS Collaboration, Report No. ATLAS-CONF-2013-062.

[36] J. Alwall, P. Schuster, and N. Toro, Phys. Rev. D 79, 075020 (2009). 
[37] D. S. M. Alves, E. Izaguirre, and J. G. Wacker, J. High Energy Phys. 10 (2011) 012.

[38] G. Aad et al. (ATLAS Collaboration), J. High Energy Phys. 10 (2013) 130.

[39] CMS Collaboration, Report No. CMS-PAS-SUSY-13-012.

[40] G. Aad et al. (ATLAS Collaboration), Phys. Rev. D 86, 092002 (2012).

[41] J. Alwall, M. Herquet, F. Maltoni, O. Mattelaer, and T. Stelzer, J. High Energy Phys. 06 (2011) 128.

[42] W. Beenakker, R. Hopker, and M. Spira, arXiv:hep-ph/ 9611232.

[43] Prospino 2, http://www.thphys.uni-heidelberg.de/ plehn/ index.php?show=prospino\&visible=tools.

[44] evchain, http://www.github.com/hep-platform/evchain.

[45] T. Sjostrand, S. Mrenna, and P. Z. Skands, J. High Energy Phys. 05 (2006) 026.

[46] T. Sjostrand, S. Mrenna, and P. Z. Skands, Comput. Phys. Commun. 178, 852 (2008).
[47] PGS4, Pretty Good Simulation of High Energy Collisions, http://physics.ucdavis.edu/ conway/research/software/pgs/ pgs4-general.htm.

[48] C. Dohmen et al. (SINDRUM II. Collaboration), Phys. Lett. B 317, 631 (1993).

[49] W. H. Bertl et al. (SINDRUM II Collaboration), Eur. Phys. J. C 47, 337 (2006).

[50] R. Kitano, M. Koike, and Y. Okada, Phys. Rev. D 66, 096002 (2002); 76,059902(E) (2007).

[51] P. Meade and M. Reece, arXiv:hep-ph/0703031.

[52] J. Alwall, A. Ballestrero, P. Bartalini, S. Belov, E. Boos, A. Buckley, J. M. Butterworth, L. Dudko et al., Comput. Phys. Commun. 176, 300 (2007).

[53] The Haskell Programming Language, http://www.haskell .org/haskellwiki/Haskell.

[54] The Glasgow Haskell Compiler, http://www.haskell.org/ghc.

[55] M. I. Gresham, I.-W. Kim, and K. M. Zurek, Phys. Rev. D 85, 014022 (2012). 